\title{
Modelling the spectral energy distribution of ULIRGs
}

\section{The energetic environment and the dense interstellar medium ${ }^{\star}$}

\author{
O. Vega ${ }^{1,2}$, M. S. Clemens ${ }^{2}$, A. Bressan ${ }^{1,2,3}$, G. L. Granato ${ }^{2}$, L. Silva ${ }^{4}$, and P. Panuzzo ${ }^{2,5}$ \\ 1 INAOE, Luis Enrique Erro 1, 72840 Tonantzintla, Puebla, Mexico \\ 2 INAF - Osservatorio Astronomico di Padova, Vicolo dell'Osservatorio, 5, 35122 Padova, Italy \\ e-mail: olga.vega@oapd.inaf.it \\ SISSA, Strada Costiera, 34131 Trieste, Italy \\ 4 INAF - Osservatorio Astronomico di Trieste, via Tiepolo 11, 34131 Trieste, Italy \\ 5 Laboratoire AIM, CEA/DSM - CNRS - Université Paris Diderot, DAPNIA/Service d'Astrophysique, Bât. 709, CEA-Saclay, \\ 91191 Gif-sur-Yvette Cedex, France
}

Received 19 October 2007 / Accepted 23 February 2008

\begin{abstract}
Aims. By using the spectral energy distribution (SED) from the near-infrared to the radio of a statistically significant number of luminous infrared galaxies we determine important physical parameters for this population of objects. In particular we constrain the optical depth towards the luminosity source, the star formation rate, the star formation efficiency and the AGN fraction. Methods. We fit the near-infrared to radio spectral energy distributions of a sample of 30 luminous and ultra-luminous infrared galaxies with pure starburst models or models that include both starburst and AGN components.

Results. We find that although about half of our sample have best-fit models that include an AGN component, only 30\% (9/30) have an AGN that accounts for more than $10 \%$ of the infrared luminosity from 8 to $1000 \mu \mathrm{m}$, whereas all have an energetically dominant starburst. Our derived AGN fractions are generally in good agreement with measurements of the mid-infrared line ratios, $\mathrm{Ne}[\mathrm{V}] / \mathrm{Ne}[\mathrm{II}]$ and O[IV]/Ne[II] by Spitzer IRS, but much lower than those derived from PAH equivalent widths or the mid-infrared spectral slope. Our models determine the mass of dense molecular gas within which active star formation takes place via the extinction required to reproduce the infrared part of the SED. Assuming that this mass is that traced by the HCN molecule, we reproduce the observed linear relation between HCN flux and infrared luminosity found previously. We also find that the star formation efficiency, as defined by the current star formation rate per unit molecular gas mass, falls as the starburst ages.

Conclusions. If the evolution of ULIRGs includes a phase in which an AGN contributes an important fraction to the infrared luminosity, this phase should last an order of magnitude less time than the starburst phase. However, we find no convincing evidence that an energetically important AGN is associated with a particular phase of the starburst. Because the mass of dense molecular gas that we derive is consistent with observations of the HCN molecule, it should be possible to estimate the mass of dense, star-forming molecular gas in such objects when molecular line data are not available.
\end{abstract}

Key words. galaxies: active - infrared: galaxies - radio continuum: galaxies - ISM: dust, extinction

\section{Introduction}

With total infrared luminosities between $10^{11}-10^{12} L_{\odot}$ and $\geq 10^{12} L_{\odot}$, respectively, Luminous and Ultraluminous Infrared galaxies, (U)LIRGs, are the most luminous objects in the local universe. Although scarce at low redshift, they may account for the bulk of all star formation activity at $z>2-3$ and dominate the far-infrared background (e.g. Blain et al. 2002).

Many of them are found in merging systems (e.g. Sanders et al. 1988), suggesting that dynamical interaction has driven gas towards the nucleus, fueling a massive starburst (SB) and/or the central massive black hole (Mihos \& Hernquist 1996).

Despite extensive investigation over the last decades, there is still considerable uncertainty as to the nature of their power source.

The observation that ULIRGs obey the same FIR-radio correlation as normal star forming galaxies (e.g. Sopp et al. 1990)

\footnotetext{
* Appendices A and B are only available in electronic form at http://www. aanda.org
}

and display a tight correlation between dense molecular gas mass and far-infrared luminosity (Gao \& Solomon 2004a), constitutes strong evidence that these sources are powered predominantly by star formation. However, ULIRGs may commonly host an AGN and the evidence that AGN and starburst luminosities are correlated over a wide range of IR luminosities (e.g. Farrah et al. 2003) renders the above issue more intriguing.

Many studies have been devoted to the quantification of the relative contributions of $\mathrm{AGN}$ and starburst (SB), but they are hindered by the large and uncertain extinction that, usually, is not negligible even at NIR wavelengths (Goldader et al. 1995; Silva et al. 1998; Murphy et al. 2001; Valdés et al. 2005).

In order to analyze the power mechanism in these sources, tracers that do not suffer large extinction have been preferred recently, such as hard X-rays, and MIR to radio diagnostics. Rigopoulou et al. (1996) argue that the weakness of the hard $\mathrm{X}$-ray luminosity seen in most ULIRGs reveals a real lack of AGN activity, because the gas column densities required to absorb its emission exceed those estimated from CO data. Similar 
conclusions have been found by Risaliti et al. (2006) who concluded that energetically important AGN are present in only $20 \%$ of their ULIRG sample.

Since the advent of ISO and Spitzer, MIR diagnostics such as the strengths of PAH emission and the $9.7 \mu \mathrm{m}$ absorption features, the high ionization lines, and the MIR continuum slope, have all been used to quantify the AGN contribution (Genzel et al. 1998; Dudley 1999; Imanishi et al. 2007; Farrah et al. 2007; Armus et al. 2007). However, in spite of a general consensus that the starburst is dominant in about $80 \%$ of ULIRGs, there are strong discrepancies in the results obtained with the different methods. This is in line with the recent finding that the strength of PAHs and the shape of the mid-infrared continuum cannot be safely used to disentangle AGN and starburst contributions because, on one hand, there is evidence that PAHs can be destroyed in strong star forming regions and, on the other, that MIR radiation can be self absorbed in a highly obscured environment (Vega et al. 2005; Weedman et al. 2005).

In the radio domain, the most direct way of distinguishing between AGN and starburst power sources in ULIRGs is to search for very compact radio continuum emission towards the nuclei. However radio sources of similar physical sizes have been identified as both AGN or a compact starburst, depending on the resolution of the observations. Nagar et al. (2003), by using $15 \mathrm{GHz}$ radio continuum data with a resolution of 150 mas, have concluded that most of the 83 ULIRGs of their sample are AGN powered because of the compactness of the radio sources detected. However, the resolution of their data corresponds to $420 \mathrm{pc}$ at the median redshift of their sample and, as a comparison, the SNe detected in the NW nucleus of Arp 220 by Smith et al. $(1998 \mathrm{a}, \mathrm{b})$ are within a region $0{ }^{\prime} 2 \times 00^{\prime} 4(75 \times 150 \mathrm{pc})$. Smith et al. find that no AGN is necessary to explain the IR luminosity in this source. As long as the brightness temperature does not exceed $10^{7} \mathrm{~K}$ the compactness of radio nuclei alone does not support an AGN hypothesis. Smith et al. (1998a,b) find that $7 / 11$ ULIRGs observed at $18 \mathrm{~cm}$ by VLBI could be modelled as pure starbursts, which would be consistent with their finding that the molecular gas mass is correlated with the radio flux on small scales.

In this paper we follow a different approach to examine the energetic environment in (U)LIRGs. Instead of considering a single spectral region, we base our study on the thorough analysis of the panchromatic spectral energy distribution (SED). Thus, we first determine the NIR - radio SED of a sample of 30 local (U)LIRGs by using archival IR photometry and spectroscopy, and new radio data by Clemens et al. (2008). Then, these data are compared with suitable models with SB and AGN components. Similar approaches have been adopted by Klaas et al. (2001) and by Farrah et al. (2003). They used starburst and AGN templates to model the SED from the NIR-submm range, and from the optical-submm range, respectively. We prefer to use models built on well calibrated star forming complexes instead of templates re-scaled to different luminosities for two reasons. Firstly, it avoids the dubious process of "re-scaling" a "template" to another luminosity. This point is well exemplified by the link between lack of PAH emission and dominance of the molecular cloud component with respect to the cirrus component (Vega et al. 2005). Secondly, the use of models may give access to a more sound physical picture of the environment within which such objects evolve. We are not only interested in the nature of the power mechanism, but also in its environment and evolution. This, in turn, can be used for the study of even more extreme phases such as those likely found at high redshift, which is one of the ultimate goals of the present investigation.
Another major difference with previous works is the inclusion of radio data in the analysis. Radio data constitute an independent constraint on the starburst strength and age. Indeed, Bressan et al. (2002) showed that deviations from the FIR/radio correlation could be used to infer the evolutionary status of a compact starburst. Such deviations are expected in bursts of short duration because, at early times (a few $\times 10^{6} \mathrm{yr}$ ), there is a deficit of synchrotron emission due to the low rate of production of core collapse supernovae. Such young sources are characterized by a FIR/radio ratio that is larger than the average for star forming objects, and by a radio slope that is flatter than the average synchrotron slope.

The power of considering also radio data was illustrated by Bressan et al. (2002) and Prouton et al. (2004). While Bressan et al. limited their comparative analysis to the case of pure starbursts, Prouton et al. (2004) have attempted a full panchromatic analysis by including also the possible contribution from the AGN. The latter authors pointed out the existence of a certain degree of degeneracy between the AGN contribution and dust optical depth of the molecular clouds, mainly arising from a poor sampling of the SED in the MIR spectral region.

In the present work we have greatly enlarged the (U)LIRG sample, from 7 (Prouton et al.) to 30 objects. We have also reconsidered and largely increased the number of observational data points for each galaxy, and consequently, our conclusions rely on much better sampled SEDs. This is particularly true for the MIR range, where for about one third of the sample we have also included the Spitzer IRS spectrum. From the theoretical side, we make use of the most recent improvements in our GRASIL SB models (see Sect. 3). These models adopt a better treatment of PAH emission and include results based on IR observations so that they allow a more realistic rendering of the emission in the mid-infrared.

The paper is organized as follows. In Sect. 2 we describe our sample selection and present the observed SEDs of our galaxies. Section 3 describes the models and the method used to obtain the best fit to the SEDs. Observed SEDs and best fit models are published in electronic form, see also Tables B.1-B.3. The results of the fits are presented in Sect. 4 and they are discussed in Sect. 5. Our conclusions are drawn in Sect. 6, while notes on individual objects are provided in Appendix A.

\section{Sample}

We study the sample of 30 (U)LIRGs presented by Clemens et al. (2008, hereafter Paper I). The selection criteria were specified in that work and are summarized as follows: (a) the galaxies are compact (U)LIRGs from Condon et al. (1991a) (i.e. brighter than $5.25 \mathrm{Jy}$ at $60 \mu \mathrm{m})$ with radio data at 3 or more frequencies, including the new measurements at $22 \mathrm{GHz}$ (Paper I). The well-sampled radio spectra will allow us to put strong constraints on the age of the burst of star formation (Bressan et al. 2002). (b) We also require the galaxies to have a well-sampled infrared SED, with data in the NIR, MIR and FIR spectral ranges. We have excluded from the analysis data at wavelengths shorter than the NIR because, due to the large extinction, they are likely to be dominated by the underlying old stellar population and not by the starburst (e.g. Surace et al. 2000; Farrah et al. 2001). Thus, the inclusion of optical data would introduce new free parameters, all related tothe geometry, ages, and metallicities of this unobscured stellar population. To a similar extent, the NIR continuum also may be dominated by the old population, but its inclusion is needed because a $J-K$ colour excess over 
Table 1. Global properties of our sample of (U)LIRGs. Galaxies marked with an * have warm IR colors, $f_{25} / f_{60}>0.2$. Galaxies marked with a ${ }^{\dagger}$ have NIR colours $J-K>2$, and those marked with a ${ }^{\S}$ have available Spitzer IRS spectra. Galaxies are ordered by decreasing total IR luminosity.

\begin{tabular}{|c|c|c|c|c|c|c|c|c|c|c|}
\hline Name & $\begin{array}{c}\log \left(L_{\mathrm{IR}}\right) \\
\left(L_{\odot}\right)\end{array}$ & $\begin{array}{c}D_{\mathrm{L}} \\
(\mathrm{Mpc})\end{array}$ & $12 / 25$ & $60 / 100$ & $J-K$ & $q_{1.4 \mathrm{GHz}}$ & $\alpha_{22}^{1.4}$ & $\begin{array}{l}\text { Spectral } \\
\text { type }\end{array}$ & $\begin{array}{l}\text { Radio } \\
\text { size (") }\end{array}$ & References \\
\hline UGC $8058^{*, \dagger, \S}$ & 12.49 & 174.0 & 0.21 & 1.06 & 2.22 & 2.14 & -0.25 & Sy1 & $\leq 0.07 \times 0.06$ & $1,2,3,4,5,6,7,8,9,10,11$ \\
\hline IR $14348-1447^{\dagger}$ & 12.30 & 351.3 & 0.19 & 0.97 & 1.71 & 2.36 & -0.75 & $\mathrm{~L}$ & $0.17 \times 0.14$ & $1,2,9,11$ \\
\hline IR $12112+0305$ & 12.28 & 309.3 & 0.17 & 0.85 & 1.22 & 2.66 & -0.51 & HII & $0.19 \times 0.10$ & $1,2,9,12,11,13$ \\
\hline IR $05189-2524^{*, \dagger, \S}$ & 12.11 & 175.7 & 0.21 & 1.20 & 2.17 & 2.74 & $(\ldots)$ & Sy2 & $0.20 \times 0.17$ & $1,2,9,11,14$ \\
\hline UGC $9913^{\S}$ & 12.11 & 73.5 & 0.06 & 0.91 & 1.18 & 2.59 & -0.46 & $\mathrm{HII} / \mathrm{L}$ & $0.32 \times 0.19$ & $1,2,9,10,11,12,13,15$ \\
\hline IR $08572+3915^{*, \S}$ & 12.09 & 243.6 & 0.19 & 1.53 & 1.90 & 3.27 & -0.11 & $\mathrm{~L} / \mathrm{Sy} 2$ & $0.09 \times 0.07$ & $1,9,11,13$ \\
\hline UGC $8696^{\S}$ & 12.09 & 155.3 & 0.10 & 1.01 & 1.30 & 2.25 & -0.83 & Sy2/L & $0.32 \times 0.18$ & $1,2,5,9,10,11,16$ \\
\hline IR $15250+3609^{\S}$ & 12.01 & 229.7 & 0.15 & 1.17 & 1.16 & 2.76 & -0.45 & $\mathrm{~L}$ & $\leq 0.06 \times 0.05$ & $1,2,9,11,12,13$ \\
\hline IR $10565+2448^{\S}$ & 11.98 & 177.9 & 0.16 & 0.80 & 1.09 & 2.44 & $(\ldots)$ & HII & $\leq 0.25 \times 0.25$ & $1,11,12,13,17$ \\
\hline UGC $5101^{\S}$ & 11.90 & 162.2 & 0.24 & 0.57 & 1.80 & 1.99 & -0.81 & L/Sy1.5 & $0.14 \times 0.11$ & $1,9,11,16,18$ \\
\hline IZW 107 & 11.83 & 165.6 & 0.15 & 0.86 & 1.11 & 2.36 & -0.95 & HII & $0.28 \times 0.25$ & $1,12,13,19$ \\
\hline IR 01364-1042 & 11.78 & 200.4 & 0.13 & 0.96 & 1.19 & 2.71 & -0.50 & $\mathrm{~L}$ & $0.19 \times 0.08$ & 1 \\
\hline IR $10173+0828$ & 11.74 & 198.9 & 0.13 & 1.07 & 1.16 & 2.84 & -0.44 & $(\ldots)$ & $\leq 0.08 \times 0.05$ & $1,12,13$ \\
\hline Arp $299^{*}$ & 11.72 & 41.5 & 0.16 & 0.97 & 1.29 & 2.09 & -0.80 & $(\ldots)$ & $0.38 \times 0.28$ & $1,15,20,21$ \\
\hline UGC 4881 & 11.65 & 161.8 & 0.21 & 0.69 & 1.15 & 2.37 & -0.85 & HII & $0.20 \times 0.15$ & 1,13 \\
\hline CGCG $436-30^{\S}$ & 11.64 & 127.8 & 0.14 & 1.11 & 1.31 & 2.40 & -0.63 & $\mathrm{~L} / \mathrm{HII}$ & $0.45 \times 0.30$ & $1,13,22$ \\
\hline IC 1623 & 11.63 & 81.4 & 0.19 & 0.74 & 1.41 & 2.08 & -0.88 & $(\ldots)$ & $\leq 0.40 \times 0.25$ & $1,19,23,24$ \\
\hline NGC $1614^{*}$ & 11.60 & 64.5 & 0.20 & 0.99 & 1.28 & 2.45 & -0.68 & HII/Sy2 & $(\ldots)$ & $1,12,13,16,18,25,26,27,28$ \\
\hline UGC $8387^{\S}$ & 11.58 & 95.0 & 0.19 & 0.61 & 0.96 & 2.32 & -0.95 & L/HII & $0.52 \times 0.33$ & $1,12,13,17,27$ \\
\hline NGC $7469^{*}$ & 11.58 & 66.0 & 0.23 & 0.77 & 1.26 & 2.29 & -0.84 & Sy1.2 & $0.16 \times 0.09$ & $1,8,13,16,29,30,31$ \\
\hline UGC 2369 & 11.57 & 127.7 & 0.17 & 0.79 & 1.12 & 2.33 & -0.80 & HII & $0.13 \times 0.13$ & $1,12,13,19,32$ \\
\hline IIIZW 35 & 11.56 & 112.0 & 0.10 & 0.94 & 1.27 & 2.58 & -0.52 & $\mathrm{~L} / \mathrm{HII}$ & $0.18 \times 0.14$ & 1,13 \\
\hline IC 5298* & 11.54 & 111.9 & 0.17 & 0.75 & 1.29 & 2.53 & -0.79 & Sy2/L/HII & $0.12 \times 0.09$ & 1,13 \\
\hline Arp 148 & 11.51 & 141.6 & 0.33 & 0.55 & 1.37 & 2.37 & -0.64 & HII & $0.22 \times 0.10$ & $1,12,13$ \\
\hline NGC $2623^{\S}$ & 11.49 & 74.8 & 0.12 & 0.83 & 1.15 & 2.49 & -0.60 & $\mathrm{~L} / \mathrm{Sy} 2$ & $0.38 \times 0.26$ & $1,12,16,28$ \\
\hline Mrk 331 & 11.44 & 74.9 & 0.19 & 0.81 & 1.24 & 2.52 & -0.70 & $\mathrm{HII} / \mathrm{Sy} 2$ & $0.12 \times 0.09$ & $1,13,16,17,27$ \\
\hline NGC 34 & 11.44 & 79.6 & 0.15 & 1.01 & 1.18 & 2.49 & -0.79 & Sy2 & $0.39 \times 0.29$ & $1,20,33$ \\
\hline NGC 5256 & 11.44 & 113.7 & 0.33 & 0.66 & 1.47 & 1.90 & -0.77 & Sy $2 / \mathrm{HII}$ & $0.25 \times 0.21$ & $1,2,13,28,29,34$ \\
\hline UGC 6436 & 11.41 & 140.1 & 0.44 & 0.57 & 1.05 & 2.65 & -0.70 & $\mathrm{~L} / \mathrm{HII}$ & $\leq 0.30 \times 0.30$ & 1,13 \\
\hline NGC 6286 & 11.16 & 74.4 & 0.50 & 0.44 & 1.36 & 1.94 & -0.95 & $\mathrm{HII} / \mathrm{L}$ & $\leq 0.25 \times 0.25$ & $1,10,17,22$ \\
\hline
\end{tabular}

References. (1) NED, IRSA, Clemens et al. (2008), (2) Klaas et al. (2001), (3) Roche \& Chandler (1993), (4) Joyce \& Simon (1976), (5) Rieke (1978), (6) Rieke (1976), (7) Joyce et al. (1975), (8) Weedman et al. (2005), (9) Rigopoulou et al. (1996), (10) Carico et al. (1992), (11) Farrah et al. (2003), (12) Dunne \& Eales (2001), (13) Dunne et al. (2000), (14) Maiolino \& Rieke (1995), (15) Chini et al. (1986), (16) Benford (1999), (17) Dale et al. (2000), (18) Carico et al. (1988), (19) Spinoglio et al. (2002), (20) Gallais et al. (2004), (21) Charmandaris et al. (2002a,b), (22) Stickel et al. (2004), (23) Frayer et al. (1999), (24) Le Floc'h et al. (2002), (25) Rieke \& Low (1972), (26) Lebofsky \& Rieke (1979), (27) Soifer et al. (2001), (28) Brandl et al. (2006), (29) Pérez-García \& Rodríguez-Espinosa (2001), (30) Alonso-Herrero et al. (2001), (31) Papadopoulos \& Allen (2000), (32) Hwang et al. (1999), (33) Albrecht et al. (2007), (34) Alonso-Herrero et al. (2003).

that expected from a pure stellar component is a strong indication of the presence of an AGN (e.g. Berta et al. 2003).

The original sample presented in Paper I is of 31 galaxies, however we exclude one of the galaxies (IRAS $03359+1523$ ) from this analysis because of confusion with a nearby source of similar strength in the far-infrared.

In Table 1 we list some general information for our sample. Column (1) displays the name of the galaxy. In Col. (2) we list the logarithm of the total $(8-1000 \mu \mathrm{m})$ IR luminosity (Sanders \& Mirabel 1996) for each galaxy. In Col. (3) we list the luminosity distances, $D_{\mathrm{L}}$, of the galaxies, calculated by assuming $H_{0}=75 \mathrm{Mpc} / \mathrm{km} / \mathrm{s}, \Omega_{\mathrm{M}}=0.3$ and $\Omega_{\Lambda}=0.7$, and the radial velocities given in $\mathrm{NED}^{1}$. Columns (4) and (5) list the $12 / 25$ and $60 / 100$ IRAS colours respectively, where all the IR data were taken from NED. Column (6) displays the

\footnotetext{
1 The NASA/IPAC Extragalactic Database is operated by the Jet Propulsion Laboratory, California Institute of Technology, under contract with the National Aeronautics and Space Administration.
}

$J-K$ colour. NIR data are 2 MASS data taken from IRSA ${ }^{2}$. In Col. (7) we list the value of the FIR to radio luminosity ratio, $q_{1.4 \mathrm{GHz}}=\log \left(\frac{\mathrm{FIR}}{3.75 \times 10^{12} \mathrm{~W} \mathrm{~m}^{-2}}\right)-\log \left(\frac{S_{1.4 \mathrm{GHz}}}{\mathrm{W} \mathrm{m}^{-2} \mathrm{~Hz}^{-1}}\right)$, and in Col. (8) the radio spectral index between 22 and $1.4 \mathrm{GHz}$, $\alpha_{22}^{1.4}=-\frac{\log \left(S_{1.4 \mathrm{GHz}} / S_{22} \mathrm{GHz}\right)}{\log (1.4 \mathrm{GHz} / 22 \mathrm{GHz})}$. In both cases, radio data were taken from Paper I. Column (9) displays the optical spectral classification of each galaxy from NED. Column (10) displays the size of the radio emitting region at $1.4 \mathrm{GHz}$ from Condon et al. (1991a).

\subsection{The NIR to radio data}

For all the sources of our sample we collected NIR data from the 2MASS All-Sky Extended Source Catalog and IRAS data from IRSA and NED, respectively. Radio data were taken from Paper I. Additional IR and sub-mm broad-band data were taken from the literature. The references to those works are quoted in

\footnotetext{
2 The NASA/IPAC Infrared Science Archive is operated by the Jet Propulsion Laboratory, California Institute of Technology, under contract with the National Aeronautics and Space Administration.
} 
the last column of Table 1 . In order to clarify which NIR to radio data were effectively used in our SED modelling, we also provide, in electronic form, the SED data for each galaxy ${ }^{3}$.

We found Spitzer IRS spectra in the SSC archive for eleven objects of our sample of (U)LIRGs: UGC 8058, IRAS 05189-2524, UGC 9913, IRAS 08572+3915, UGC 8696, IRAS 15250+3609, IRAS 10565+2448, UGC 5101, UGC 8387, CGCG 436-30, and NGC 2623 (project IDs 105, and 14, P.I.: J. R. Houck, and project ID 30323, P.I.: L. Armus). In order to include them in the fitting procedure, we calculated from the Spitzer spectra the values of the specific fluxes at 5.8 and $10 \mu \mathrm{m}$, those in the LW2 and LW3 ISO bands and those in the IRAS 12 and 25 microns bands, by taking into account the suitable filter responses. We excluded the fluxes in the Spitzer IRAC 3 $(5.7 \mu \mathrm{m})$ and IRAC $4(8 \mu \mathrm{m})$ bands because these are too dominated by molecular absorption bands (e.g. Lahuis et al. 2007), which are not included in our models.

In the compilation of fluxes care was taken to avoid confusion with nearby sources and, in the case of interferometric radio data, losses due to high resolution mapping.

Our objects are compact, with the radio emitting regions ranging from $290 \times 239 \mathrm{pc}$ for IR $14348-1447$ to $43 \times 33 \mathrm{pc}$ for Mrk 331 (see Col. 10 in Table 1). They are also isolated so that we are confident that our fluxes sample the entire starburst in all cases.

\section{Models}

In this section we describe the model that will be used to interpret the SEDs of our galaxy sample.

As already mentioned, there is considerable evidence that the starburst component is always present and possibly dominates the bolometric luminosity in luminous and ultra-luminous IRAS galaxies. Therefore, we adopt the following approach to find the best fit model. We first test the null-hypothesis that our sample sources are pure starbursts. Then, if the observed SED shows an excess emission over the best fit in those wavelength regions that are more affected by the presence of an embedded AGN, typically in the NIR-FIR range, we reject the null-hypothesis and add an AGN component to the model.

The starburst models (SB) are selected from a library generated with GRASIL ${ }^{4}$ (Silva et al. 1998) and involve seven main free parameters that will be discussed below.

The best fit is obtained by minimizing the merit function, $\chi$, calculated as

$\chi=\frac{1}{N} \sum_{i=1}^{N}\left(\frac{F_{\bmod }(i)-F_{\text {obs }}(i)}{\operatorname{Err}(i)}\right)^{2}$

where $F_{\text {mod }}(i), F_{\text {obs }}(i)$, and $\operatorname{Err}(i)$ are the model flux values, the observed fluxes and observational errors respectively. $N$ is the number of passbands used for the fit and it is always greater than the number of parameters of the starburst model (i.e. 7, see Sect. 3.2). Thus, the best fit SB model is always wellconstrained. We consider that a satisfactory best fit starburst model is reached when, in all passbands, the residuals between observations and models are less than the typical observational error of the passband.

\footnotetext{
3 These can also be downloaded from the webpage: http:// adlibitum. oats. inaf.it/silva/default.html

4 GRASIL can be run via the WEB interface web.pd.astro.it/ galsynth/ which is maintained at OAPD, INAF by L. Paoletti, A. Petrella \& D. Selvestrel, and can also be downloaded from the webpage http://adlibitum. oats . inaf. it/silva/default. html
}

However, in some cases, the residuals between the observations and the SB model in one or more passbands, mainly in the NIR/MIR range, are larger than the typical error of the corresponding flux. Then we reject our null-hypothesis and consider the possibility that an AGN component, characterized by a hotter dust emission, is present. The AGN model is selected from a library generated with the radiative transfer code developed by Granato \& Danese (1994) and added to the SB model in such a way that the total luminosity is

$L_{\lambda}^{\mathrm{tot}}=(1-f) \times L_{\lambda}^{\mathrm{SB}}+f \times L_{\lambda}^{\mathrm{AGN}}$, with $0 \leq f \leq 1$.

The best fit is again searched for by using the merit function given by Eq. (1). Since the AGN models are characterized by another seven free parameters, one could question whether the fit is still well-constrained because the total number of free parameters $(\mathrm{SB}+\mathrm{AGN})$ may exceed the number of observed passbands. Actually, anticipating our results, this is the case for one object, IRAS 01364-1042, where the number of adopted passbands is 11 and the estimated contribution of the AGN amounts to $\sim 10 \%$. However, as will be discussed below, we could have simply estimated the AGN contribution as the flux excess over the best fit SB model.

A more critical point is concerned with the radio emission of the AGN which is not considered in the model by Granato \& Danese (1994). Following Prouton et al. (2004), we assume that the contribution from the AGN to the radio emission is negligible so that the radio emission is entirely ascribed, and actually becomes a strong constraint on, the SB component. This assumption is supported by VLBI observations of the majority of our sources, indicating an average value of $S_{\mathrm{VLBI}} / S_{\mathrm{Tot}}=0.12$ at $1.6 \mathrm{GHz}$, thus suggesting that the contribution of the AGN to the radio can indeed be neglected (Smith et al. 1998a,b).

In the followings subsections, we briefly describe the library of SB and AGN models.

\subsection{Starburst models: a brief description of GRASIL}

GRASIL is a population synthesis code which predicts the SED of galaxies from the far-UV to the radio, including state-ofthe-art treatment of dust reprocessing (Silva et al. 1998; Silva 1999; Granato et al. 2000), production of radio photons by thermal and non-thermal processes (Bressan et al. 2002), nebular line emission (Panuzzo et al. 2003) and an updated treatment of PAH emission (Vega et al. 2005). We refer the reader to the original papers for details, but we summarize here, for convenience, the features most relevant to the present work.

The star formation history of a galaxy with a burst of star formation is computed in the GRASIL chemical evolution code by adopting a Schmidt-type law, $\operatorname{SFR}(t)=v_{\mathrm{Sch}} M_{\mathrm{gas}}(t)^{k}$, of efficiency $v_{\mathrm{Sch}}$, and exponent $k$. The burst of star formation is simulated by superposing on this quiescent component a starburst phase, characterized by an exponentially decreasing star formation rate, with $e$-folding time " $t_{\mathrm{b}}$ " and age "age $e_{\mathrm{b}}$ ". In order to quantify the number of massive stars, the code adopts an initial mass function (IMF). The chemical evolution code determines, as a function of the galaxy age $\left(T_{\mathrm{gal}}\right)$, the star forming rate $(S F R)$, the supernova rate (SNR), the number of stars of a given mass and metallicity, the remaining mass of gas $\left(M_{\mathrm{gas}}\right)$, and the total baryonic mass of the galaxy $\left(M_{\mathrm{T}}\right)$.

Then, GRASIL computes the interaction between the stellar radiation and dust using a relatively realistic and flexible geometry for both stars and dust. In general, the system is described as a superposition of an exponential disk component and a bulge 
component, the latter modelled by a King profile. However, for the starburst models, we consider that the spherically symmetric King profile alone, with core radii corresponding to the radial scale lengths for the star and dust components, $r_{\star}, r_{\text {dust }}$, and identical spatial dependence, i.e. $r_{\star}=r_{\text {dust }}=r$, is a reasonably good approximation (Silva et al. 1998).

One of the most novel features of GRASIL is that the ISM is divided into two phases, the diffuse ISM, corresponding to cirrus dust, and the much denser molecular cloud component (MCs). The code performs the radiative transfer of starlight through the MCs and diffuse ISM, by taking into account that new stars are born inside MCs and progressively lose them (either because they escape or because the clouds are destroyed). This process leads to an age-dependent extinction of stellar generations, in the sense that the younger stars are more affected by dust obscuration in MCs. This is approximately described in GRASIL as follows. If $t_{\mathrm{esc}}$ is the timescale for the process, the fraction of starlight radiated inside the clouds at time $t$ after they formed is given by

$F(t)= \begin{cases}1 & \left(t \leq t_{\mathrm{esc}}\right) \\ 2-\frac{t}{t_{\mathrm{esc}}} & \left(t_{\mathrm{esc}}<t \leq 2 t_{\mathrm{esc}}\right) \\ 0 & \left(t>2 t_{\mathrm{esc}}\right) .\end{cases}$

In practice, $100 \%$ of the stars younger than $t_{\mathrm{esc}}$ are considered to radiate inside the MCs, and this percentage decreases linearly to $0 \%$ in $2 t_{\text {esc }}$. The timescale $t_{\text {esc }}$ is a fundamental parameter which is found to be larger in starburst than in normal disk-like galaxies (Silva et al. 1998). The mass of gas involved in each component is specified by the parameter $f_{\mathrm{mc}}$, which represents the relative fraction of gas in the molecular cloud phase. Then, the total molecular mass, $M_{\text {mol }}=f_{\mathrm{mc}} \times M_{\text {gas }}$, is subdivided into spherical clouds whose optical depth, $\tau_{1}$, is another important free parameter. In general, GRASIL computes the mass of dust by using a gas-to-dust mass ratio, $G / D$. This approach assumes that gas and dust are well mixed and have the same spatial distribution. For the intrinsic dust properties, GRASIL adopts a mixture of graphite and silicate grains and PAHs with continuous size distribution. Here, as in other previous works (see Silva et al. 1998; and Vega et al. 2005, for more details), the dust parameters are set to match the extinction and emissivity properties of the local ISM.

The simple stellar populations (SSPs) included in GRASIL are based on the Padova stellar models, and cover a wide range of ages and metallicities. Starlight reprocessing from dust in the envelopes of AGB stars is included directly into the SSPs, as described by Bressan et al. (1998).

\subsection{The library of starburst SEDs}

Our starburst library spans a wide range of parameters: star formation history, obscuration times, dust opacities, etc. The underlying quiescent star formation history is fixed using a Schmidttype law for star formation with an efficiency of $v_{\mathrm{Sch}}=0.5 \mathrm{Gyr}^{-1}$ and an exponent $k=1$, with an infall of primordial gas on a timescale of $t_{\text {inf }}=9$ Gyr. For the IMF, we have adopted a Salpeter IMF with slope $x=1.35$ and $M_{\text {low }}=0.1 M_{\odot}$ to $M_{\text {up }}=100 M_{\odot}$. To mimic the starburst, we superimposed, at a late time $(11.95 \mathrm{Gyr})$, an exponential burst with different $e$-folding times, $t_{\mathrm{b}}$. The strength of the burst was fixed by imposing the constraint that the mass of stars formed during the burst was $10 \%$ of the gas mass at that epoch. We then follow the evolution of the SED models at different ages $\left(a e_{b}\right)$ from the early starburst phase to the post-starburst and quiescent-normal phase (see Vega et al. 2005, for a description of those phases). For the computation of non-thermal radio emission we assumed that the lower limit of initial mass giving rise to a type II SN is $8 M_{\odot}$ (Portinari et al. 1998; Bressan et al. 2002b).

Among the GRASIL parameters that mostly affect the IRradio range we recall the optical depth of MCs (parameterized via $\tau_{1}$ i.e. at $1 \mu \mathrm{m}$ ), the mass-fraction of dense molecular gas to total gas mass $\left(f_{\mathrm{mol}}\right)$, the escape time of newly born stars from their parent MCs $\left(t_{\mathrm{esc}}\right)$, and the gas-to-dust ratio $(G / D)$. Since there is evidence that the metallicity in this kind of galaxies is slightly super-solar (Rupke et al. 2007), we assumed a conservative value for the metallicity of the gas, $Z \sim 1.1 Z_{\odot}$, and a $G / D \propto 1 / Z$, with the proportionality constant chosen to have $G / D=110$ for $Z=Z_{\odot}$ (Dwek 1998).

For the spatial distribution of stars and dust, we adopted a spherically symmetric King profile (Silva et al. 1998) where the King profile core radius $r$ was allowed to vary by the typical sizes of compact starburst regions. The last free parameter that we considered in our models was the sub-mm dust emissivity index, $\beta$. Theoretical studies indicate $\beta$ between 1.5 to 2 but infrared SEDs fitted with single or double modified black bodies require values of $\beta$ as low as $\beta \simeq 0.7$ (e.g. Dunne et al. 2000). Instead when the dust temperature distribution is properly computed as in GRASIL, fits to similar sources require $\beta>1.5$, consistent with theoretical predictions.

In summary our starburst models are characterized by the following free parameters, whose ranges of values are listed in Tables 2 and 3: the $e$-folding time and age of the burst $t_{\mathrm{b}}$ and age $_{\mathrm{b}}$, the sub-mm dust spectral index $\beta$, the escape time $t_{\mathrm{esc}}$, the MC optical depth at $1 \mu \mathrm{m}, \tau_{1}$, the fraction of gas in MCs $f_{\mathrm{mc}}$, and the core radius of the King profile for the dust-star distributions $r$.

\subsection{AGN models}

A complete description of the AGN models and the numerical method developed to compute the emitted SED can be found in Granato \& Danese (1994) and Granato et al. (1997). Only the basic features are summarized here.

Several papers compared the observed IR SED of AGN with predictions from radiative transfer models within dusty tori, in order to investigate the validity of the unified model and constrain possible geometries for the obscuring structure (e.g. Pier et al. 1993; Granato et al. 1994; Efstathiou et al. 1995; Granato et al. 1997; Alonso-Herrero et al. 2001; and Nenkova et al. 2002).

The Granato \& Danese (1994) code solves, through an iterative numerical scheme, the transfer equation of the radiation that originates from the central optical-UV source, in a general axisymmetric dust distribution. The dust is composed of a mixture of grains, in thermal equilibrium with the radiation field, and extends out to a maximum radius $r_{\mathrm{m}}$.

In the absence of precise physical information concerning the structure of the obscuring torus, several geometries - and associated free-parameters - are plausible and indeed have been investigated in the papers quoted above (flared discs, tapered discs, cylinders, with and without substantial clumping etc.).

A problem which is usually overlooked in this kind of study is that dust optical properties around AGN are likely to be peculiar to some extent. Indeed, Maiolino et al. (2001a,b) presented evidence for "anomalous" properties of dust in AGN. Here the term "anomalous" is relative to the standard properties of dust grains thought to be responsible for the average Milky Way extinction law and cirrus emission. It is not surprising that the 
properties of dust grains might be very different in the dense and extreme environment of an AGN. In particular, a dust distribution that is biased in favor of large grains, ranging up to $1-10 \mu \mathrm{m}$, much larger than the usual cut at $\sim 0.3 \mu \mathrm{m}$ of Mathis (1977) type models (e.g. Draine 1984; Silva et al. 1998), has been invoked.

Thus, we have adopted several different geometries, but also investigated models in which the size distribution of grains extends to radii, $a_{\max }$, larger than the standard value. Of course, the uncertainty on optical properties of dust further limits the already moderate success of this approach in constraining the geometry (see below).

The SED fitting is performed through comparison with libraries of models. Each library consists of several hundreds of models belonging to a given "geometry class", in which typically 4-5 parameters are varied assigning 3-4 different values to them over quite a wide range. In particular, we have considered anisotropic flared discs (for definitions, see Efstathiou et al. 1995). The geometry consists of a structure whose height above the equatorial plane $h$ increases linearly with the distance in the equatorial plane $r$ (Fig. 1a in Efstathiou et al. 1995). Therefore the dust-free region is exactly conical, with a half-opening angle $\Theta_{\mathrm{h}}$. We also introduce (at variance to Efstathiou 1995) a dependence of the dust density $\rho$, and therefore of the radial optical depth, on the polar angle $\Theta$. The general form used for this dependence and that on the spherical coordinate $r$ is:

$\rho(r, \Theta) \propto r^{-\beta} \exp \left(-\alpha \cos ^{2} \Theta\right)$

where $\alpha$ and $\beta$ are model parameters.

The two additional parameters used to fully characterize the models are the ratio between the outer and inner (i.e. sublimation) radii $r_{\mathrm{m}} / r_{0}$, and the equatorial optical depth to the nucleus at $9.7 \mu \mathrm{m}, \tau_{\mathrm{e}, 9.7}$

The parameter values used in the libraries are:

$r_{\mathrm{m}} / r_{0}=30,100,300,1000,3000$

$\beta=0,0.5,1$

$\Theta_{\mathrm{h}}=20,30,40^{\circ}$

$\alpha=0,3,6$

$a_{\max }=0.3,0.5,1.0 \mu \mathrm{m}$

$\tau_{\mathrm{e}, 9.7}=1,3,10$.

All possible combinations of parameter values are considered in the libraries. For each corresponding model we compute the SED for viewing angles from $0^{\circ}$ to $90^{\circ}$ in steps of 10 degrees.

The impact of changing various parameters on the predicted SED has been explored in Granato et al. (1994, 1997). Here we recall only that, roughly speaking, $r_{\mathrm{m}} / r_{0}$ is related to the width of the IR bump, whilst $\tau_{\mathrm{e}, 9.7}$ controls mainly the NIR slope of the SEDs, as observed from obscured directions, as well as its anisotropy.

Indeed, the global result of the various work is that the nuclear SED alone does not allow strong constraints on the precise geometry of the torus. Several individual analyses have been too optimistic in this respect, essentially due to an insufficient exploration of the parameter space. Some constraints can be put on the torus extension and on the optical thickness, and good fits are obtained with flared disc models.

However, in this paper our aim is just to assess the possible contribution of the warm AGN component to the total SED of ULIRGs, without any pretense of discriminating the precise torus characteristics.
Table 2. Parameters for the star formation history of the model library. $M_{\text {low }}$ and $M_{\text {up }}$ are the adopted mass limits of the Salpeter IMF; $v_{\text {Sch }}$ is the efficiency of the Schmidt-type SFR; $\% M_{\mathrm{b}}$ is the percentage of the mass of gas involved in the burst at a galaxy age of $11.95 \mathrm{Gyr} ; t_{\mathrm{b}}$ indicates the $e$-folding time of the exponential burst superimposed on the quiescent star formation and age $e_{b}$ is the time, measured from the beginning of the burst, at which the SEDs are computed.

\begin{tabular}{cccccc}
\hline \hline$M_{\text {low }}$ & $M_{\text {up }}$ & $v_{\mathrm{Sch}}$ & $\% M_{\mathrm{b}}$ & $t_{\mathrm{b}}$ & $\log \left(\mathrm{age}_{\mathrm{b}}\right)$ \\
$M_{\odot}$ & $M_{\odot}$ & $\left(\mathrm{Gyr}^{-1}\right)$ & $M_{\odot}$ & $(\mathrm{Myr})$ & $(\mathrm{yr})$ \\
\hline 0.1 & 100 & 0.5 & 10 & $5-80$ & $6.0-8.5$ \\
\hline
\end{tabular}

Table 3. GRASIL parameters for the starburst SED library. $G / D$ is the gas to dust mass ratio; $\beta$ is the dust emissivity index; $t_{\mathrm{esc}}$ is the escape time of newly born stars from their parent MCs; $f_{\text {mol }}$ is the fraction of gas in MCs; $\tau_{1}$ is the $1 \mu \mathrm{m}$ optical depth of MCs, and $r$ is the core radius of the King profile for the dust-star distributions.

\begin{tabular}{cccccc}
\hline \hline$G / D$ & $\beta$ & $\begin{array}{c}t_{\mathrm{esc}} \\
(\mathrm{Myr})\end{array}$ & $\tau_{1}$ & $f_{\mathrm{mol}}$ & $\begin{array}{c}r \\
(\mathrm{kpc})\end{array}$ \\
\hline 100 & $1.5-2$ & $5-80$ & $10-180$ & $0.05-0.9$ & $0.01-1$ \\
\hline
\end{tabular}

\section{Results}

\subsection{Model fits}

The good spectral coverage from the NIR to the radio of our sample imposes strong constraints, not only on the global properties of the galaxies, i.e. SFR, but also on other important physical parameters. In the absence of an AGN, the fit to the total IR and radio luminosities is related to the star formation rate, while the detailed shape of the SED is affected mainly by the optical depth of the dust $\left(\tau_{1}\right)$, the escape time $\left(t_{\text {esc }}\right)$, the age of the burst $\left(\mathrm{age}_{\mathrm{b}}\right)$, the $e$-folding time of the burst $\left(t_{\mathrm{b}}\right)$, the fraction of gas in molecular clouds $\left(f_{\mathrm{mc}}\right)$ and the sub-mm dust spectral index $(\beta)$.

The optical depth mainly affects the MIR spectral range by varying the contribution of the $\mathrm{MC}$ emission in the MIR. $t_{\text {esc }}$ affects the NIR region, the PAH emission, and FIR colour $\left(f_{60} / f_{100}\right)$ by changing the contribution of unextinguished young red supergiants to the NIR, by the heating of the cirrus from un-extinguished young stars, and by the heating of MCs from extinguished young stars, respectively. Age $\mathrm{e}_{\mathrm{b}}$ affects the radio spectral index, and in combination with $t_{\mathrm{b}}$, it has also been used to parameterize the evolutive phase of the starburst as $E_{\mathrm{SB}}=\mathrm{Age}_{\mathrm{b}} / t_{\mathrm{b}}$. Higher values of $E_{\mathrm{SB}}$, indicating more evolved starbursts, are associated with higher NIR fluxes and cooler FIR colour (see Vega et al. 2005, for a thorough discussion). $f_{\mathrm{mc}}$ affects the FIR colour and the sub-millimetre region, while $\beta$ mainly affects the sub-millimetric region.

Summarizing, earlier evolutionary phases, longer escape times and lower dust masses in MCs combine to heat the molecular clouds producing warmer FIR colour. The sub-millimetre spectral region provides information on the dust emissivity index, and also on the total dust mass, because in this region emission from the MCs and from the diffuse components are comparable. The form of the radio spectrum is defined mainly by the age of the starburst, in the sense that younger objects have flatter radio slopes.

The presence of an AGN may also change the shape of the SED by adding an extra warm component. Typical evidence of AGN in our observed SEDs is the presence of a near power-law shape in the NIR to FIR spectral region. 

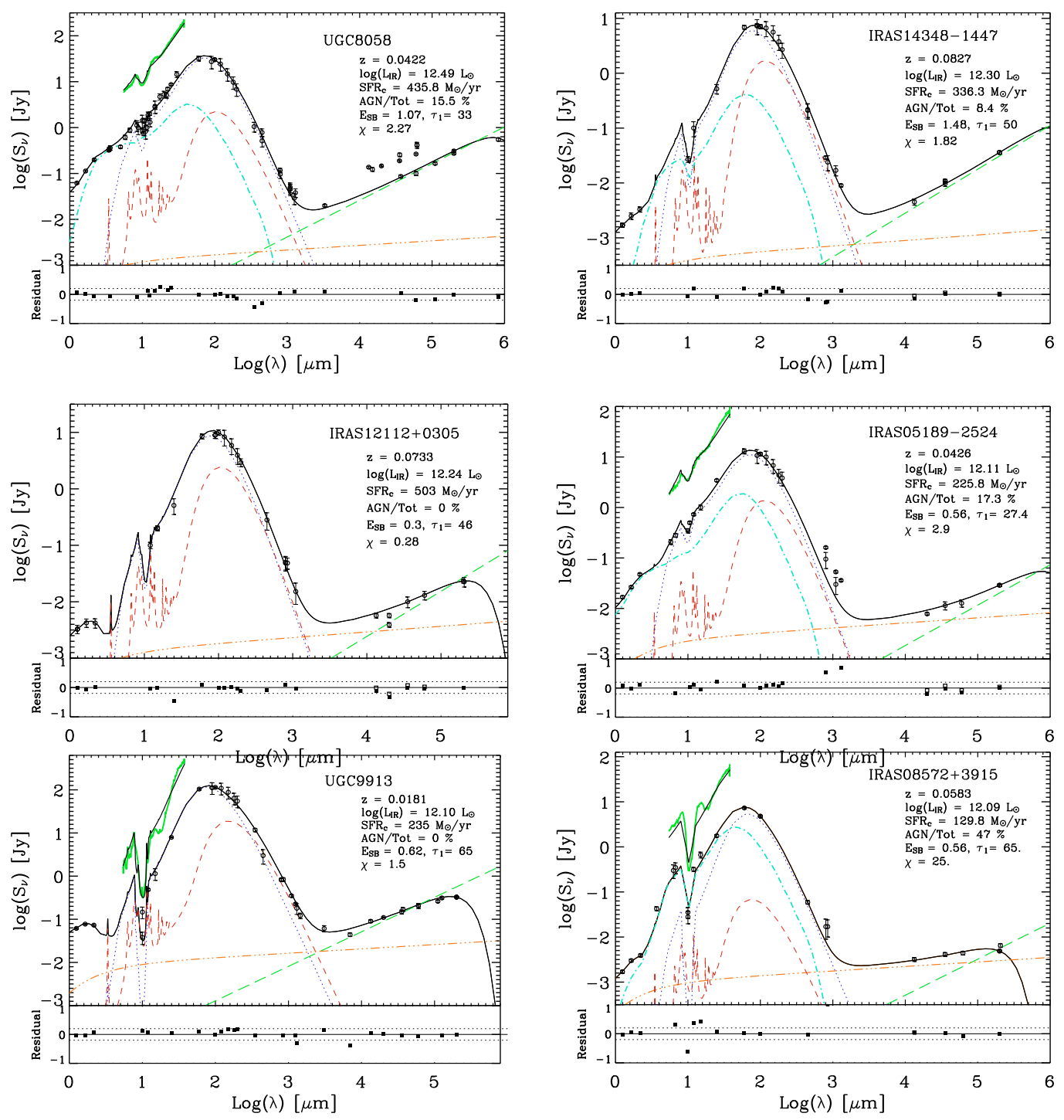

Fig. 1. Comparison between the broad band SED (open circles) and the best fit model (thick solid line). The different emission components of the starburst are: the diffuse medium (short dashed line), the molecular clouds (dotted line), thermal radio emission (three dots-dashed line), and the non-thermal radio emission (long dashed line). The additional emission from the AGN, when needed, is indicated by a dot-dashed line. When available, the IRS low resolution spectrum (solid thick line) and the corresponding SED model (solid thin line) are shown upward displaced by one dex. The residuals between models and data are shown in the bottom panels as $\left(f_{\text {data }}-f_{\text {model }}\right) / f_{\text {data }}$. The thin dotted lines in the residual plots represent $\pm 20 \%$ of the residual values. The filled squares represent the residuals and their wavelengths indicate the data used in the minimization procedure. The value of the merit function, $\chi$, for the best fit is displayed in each plot. See Sect. 4 and Tables 5 and 6, for more details.

In Fig. 1 we show the full SED fits for the galaxies of our sample. The corresponding best-fit parameters of the starburst components are shown in Table 5. The last three columns of the table display the value of the evolutionary phase of the starburst, $E_{\mathrm{SB}}$, the logarithm of the IR $(8-1000 \mu \mathrm{m})$ luminosity integrated over the SB component of the SED, and the fractional contribution of the AGN to the $8-1000 \mu \mathrm{m}$ flux indicated as AGN/Tot.

Some important physical quantities derived from the best fit models are listed in Table 6. In Col. (2) we show the required free-free opacity at $1.4 \mathrm{GHz}, \tau_{\mathrm{ff}}$, to fit the $1.4 \mathrm{GHz}$ data, i.e. $f_{149 \mathrm{GHz}}^{\text {obs }}=f_{1.49 \mathrm{GHz}}^{\text {mod }} \times \mathrm{e}^{-\tau_{\mathrm{ff}}}$. Column (3) lists the $\%$ fraction of the total energy of the starburst reprocessed by the cirrus component, $C$; Cols. (4)-(8), show the logarithms of the current total mass of gas (i.e. $\mathrm{MC}+$ cirrus), $M_{\mathrm{gas}}$; the total mass of dust, $M_{\mathrm{D}}$, calculated as $M_{\mathrm{D}}=M_{\text {gas }} /(G / D)$; the mass of gas in the MC component, $M_{\text {den }}$, calculated from the parameter $f_{\mathrm{mol}}$ as $M_{\mathrm{den}}=f_{\mathrm{mol}} \times M_{\mathrm{gas}}$, the mass converted in stars during the burst, $M_{\star}^{\mathrm{b}}$, and of the final baryonic galaxy mass, $M_{\mathrm{T}}$, calculated as $M_{\mathrm{gas}}+M_{\mathrm{D}}+M_{\star}^{\text {tot }}$, respectively. By construction, the SFR in our starburst models is a decreasing function of time, with a characteristic $e$-folding time $t_{\mathrm{b}}$ of the order of a few tens of Myr. Therefore, the SFR may change considerably during the age of the burst. We show in Cols. (9) and (10) respectively, the SFR averaged over the entire burst, $\langle S F R\rangle$, and the SFR in the last $10 \mathrm{Myr}$, i.e. the current star forming rate $S F R_{\mathrm{c}}$, as generally probed by line emission diagnostics. Notice that non-thermal emission probes typical lifetimes of the SNII production, $\sim 50$ Myr. Finally, the percentage of model thermal emission to the $22 \mathrm{GHz}$ flux is given in Col. (11).

The last rows in Tables 5 and 6 list the estimate of the errors on the parameters. To estimate the errors we have assumed that the SED of UGC 9913 is representative of the starburst component of our sample of (U)LIRGs. We then calculated several simulated SEDs for UGC 9913, by randomly varying the 

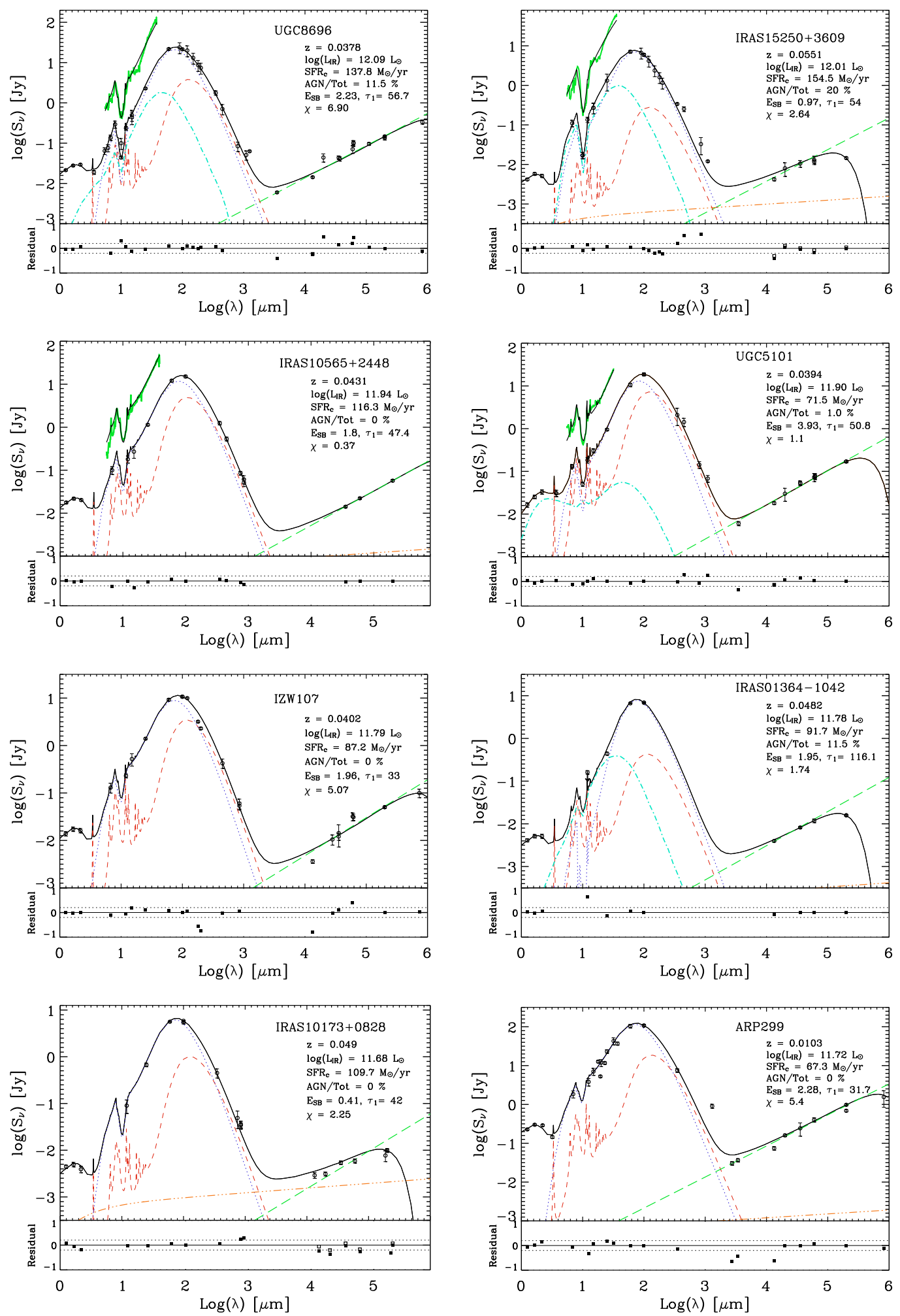

Fig. 1. continued.

observational data by \pm the corresponding observational error and, for each new SED, performed a new fit. The fractional errors on the parameters were then estimated as the ratio between their range of values and their mean value:

error $^{\text {par }}=\frac{\text { Value }_{\max }^{\text {par }}-\text { Value }_{\min }^{\text {par }}}{\left\langle\text { Value }^{\text {par }}\right\rangle}$.

Figure 2 shows, as an example, three fits to UGC 9913 obtained in this way. The largest values of the errors are derived for the total mass of gas and dust $\left(M_{\text {gas }}, M_{\mathrm{D}}\right)$ because the emission in these objects is dominated by the molecular component while the cirrus component is not well constrained. The errors for the other parameters are typically $<25 \%$.

In Table 7, we list the values of the 7 parameters for the best-fit AGN models for the 16 galaxies with emission excesses 

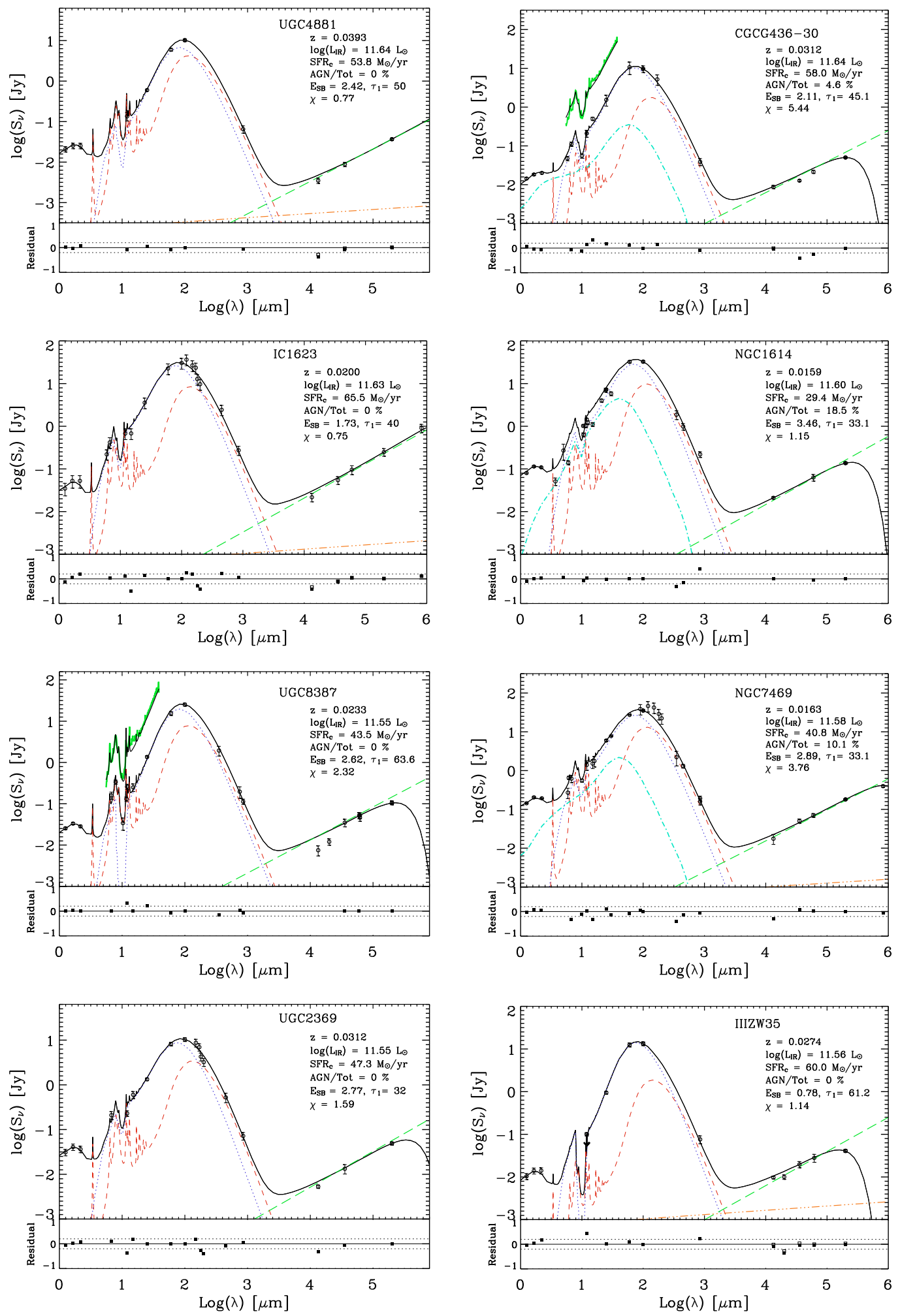

Fig. 1. continued.

that could not be fitted by the SB model alone. As already anticipated, one of the galaxies, IRAS 01364-1042, required an AGN contribution of $11.5 \%$, while the number of passbands was eleven. However, the scope of this work is not the physical characterization of the AGN component but the contribution of the AGN to the luminosity expressed as an excess over the SB luminosity. In Table 8, we show the contribution of the AGN emission to the luminosity in different IR spectral ranges. In

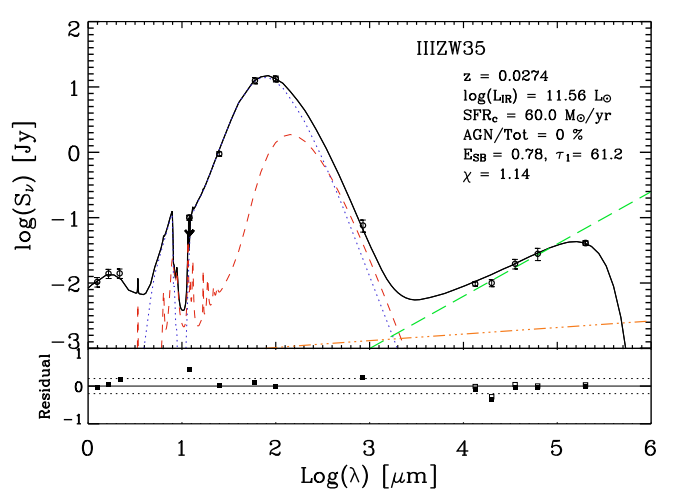

particular, the spectral ranges between $8-1000 \mu \mathrm{m}, 3-1100 \mu \mathrm{m}$, and $40-500 \mu \mathrm{m}$ are those used in different calibrations for the total IR luminosity (Takeuchi et al. 2005). In Table 9 we show the flux and the corresponding AGN contribution in 2MASS and Spitzer bands. These fluxes were calculated on the SED by using the appropriate filter responses. For the galaxies with Spitzer spectra the lower row provides fluxes calculated on the observed spectra in the IRAC 3 and IRAC 4 and MIPS $24 \mu \mathrm{m}$ 

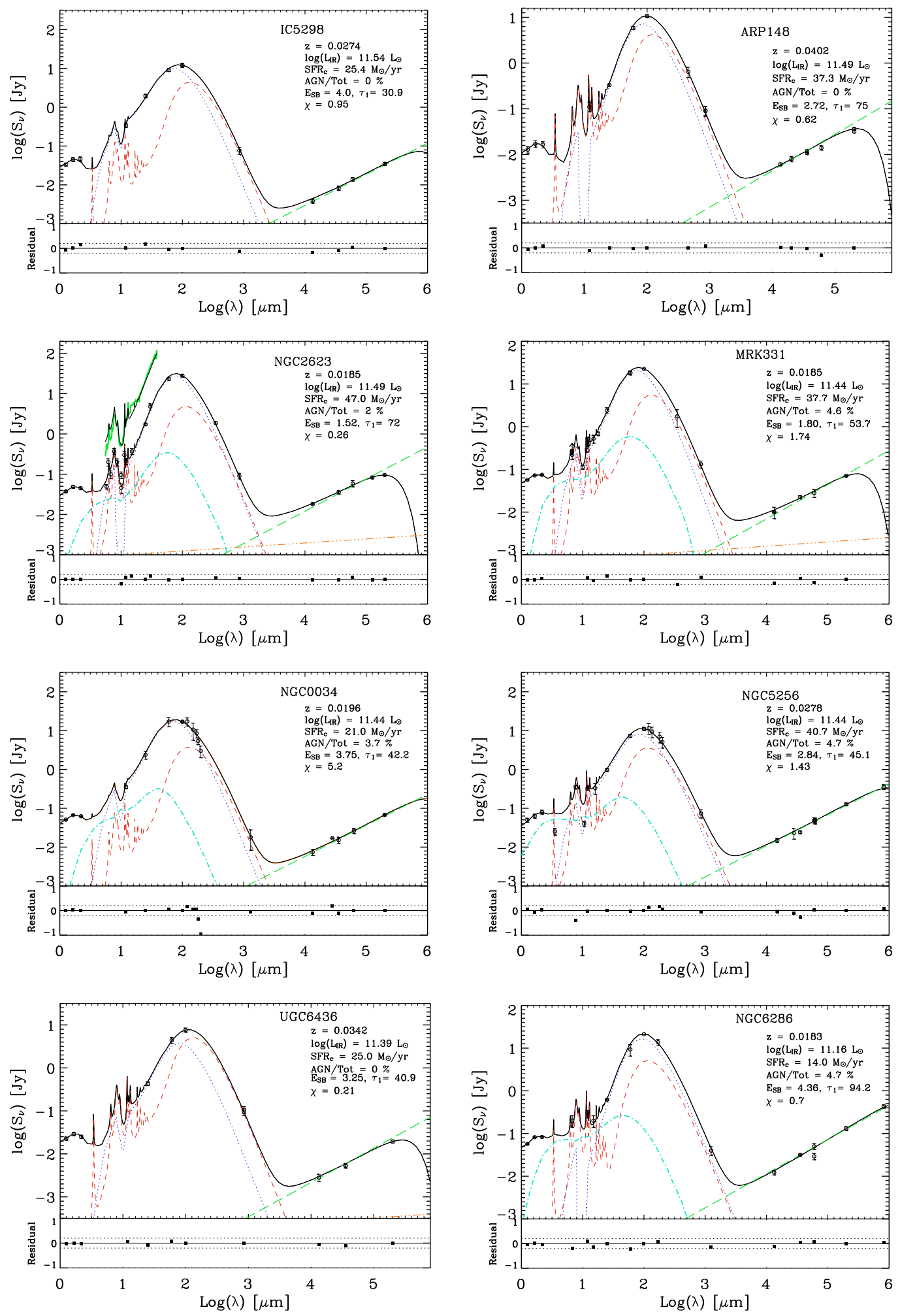

Fig. 1. continued.

bands. In the IRAC bands, differences to models in the upper row are mainly attributable to effects of molecular absorption bands (Lahuis et al. 2007). The maximum AGN contribution is always found in the NIR and MIR spectral range. It was already known both from theoretical studies (e.g. Granato et al. 1997) and direct observations (e.g. Alonso-Herrero et al. 2001) that the AGN radiation field can heat the dust to $1000-1500 \mathrm{~K}$,

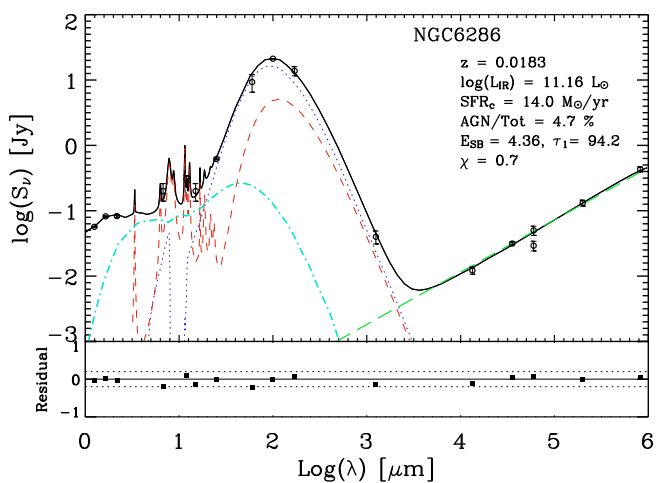

resulting in a dust thermal emission peaking in the NIR to MIR range. Moreover, all galaxies in our sample with $J-K \geq 1.7$ (see Table 8) cannot be modelled with pure SB and have AGN contributions to the $1-5 \mu \mathrm{m}$ region higher than $50 \%$. However, the presence of $J-K$ colours as blue as those typical of SB galaxies, cannot be used to discard the possible presence of an important AGN contribution to the IR luminosity. This is, for 
Table 4. List of parameter values for the AGN model library. $\Theta$ is the viewing angle, $r_{\mathrm{m}} / r_{0}$ is the maximum radius of the torus, $\alpha$ and $\beta$ are model parameters of the dust density distribution; $\Theta_{\mathrm{h}}$ is is the half opening angle of the dust free region; $a_{\max }$ is maximum radius of the dust grain, and $\tau_{\mathrm{e}, 9.7}$ is the equatorial optical thickness of the dust in the torus.

\begin{tabular}{ccccccc}
\hline \hline $\begin{array}{c}\Theta \\
(\mathrm{deg})\end{array}$ & $r_{\mathrm{m}} / r_{0}$ & $\beta$ & $\begin{array}{c}\Theta_{\mathrm{h}} \\
(\mathrm{deg})\end{array}$ & $\alpha$ & $\begin{array}{c}a_{\max } \\
(\mu \mathrm{m})\end{array}$ & $\tau_{\mathrm{e}, 9.7}$ \\
\hline $1-90$ & $30,100,300,1000,3000$ & $0,0.5,1$ & $20,30,40$ & $0,3,6$ & $0.3,0.5,1$ & $1,3,10$ \\
\hline
\end{tabular}

Table 5. Best fit parameters for the starburst model, see Sect. 4 for details. $E_{\mathrm{SB}}=\mathrm{Age}_{\mathrm{b}} / t_{\mathrm{b}}$ is the parameter which quantifies the evolutive phase of the SB. $\log \left(L_{\mathrm{IR}}^{\mathrm{SB}}\right)$ is the total $(8-1000 \mu \mathrm{m})$ IR luminosity calculated by integrating the starburst component of the SED model. $L_{\mathrm{AGN}} / L_{\mathrm{Tot}}$ is the percent contribution of the AGN to the IR luminosity. Galaxies marked with an ${ }^{*}$ have warm IR colors, $f_{25} / f_{60}>0.2$. Galaxies marked with a ${ }^{\dagger}$ have NIR colours $J-K>1.7$, and those marked with a ${ }^{\S}$ have available Spitzer IRS spectra. The last row gives an estimate of the errors on the parameters as described in Sect. 4.

\begin{tabular}{|c|c|c|c|c|c|c|c|c|c|c|}
\hline Name & $\begin{array}{c}t_{\mathrm{b}} \\
(\mathrm{Myr})\end{array}$ & $\begin{array}{c}\log \left(\operatorname{age}_{\mathrm{b}}\right) \\
(\mathrm{yr})\end{array}$ & $\beta$ & $\begin{array}{c}t_{\mathrm{esc}} \\
(\mathrm{Myr})\end{array}$ & $\tau_{1}$ & $f_{\mathrm{mol}}$ & $\begin{array}{c}r \\
(\mathrm{kpc})\end{array}$ & $E_{\mathrm{SB}}$ & $\begin{array}{c}\log \left(L_{\mathrm{IR}}^{\mathrm{SB}}\right) \\
\left(L_{\odot}\right)\end{array}$ & $\begin{array}{c}L_{\mathrm{AGN}} / L_{8-1000} \\
\% \\
\end{array}$ \\
\hline UGC $8058^{*, \dagger, \S}$ & 35 & 7.572 & 2.00 & 26 & 33.13 & 0.65 & 0.40 & 1.07 & 12.35 & 16 \\
\hline IR $14348-1447^{\dagger}$ & 10 & 7.170 & 1.95 & 14 & 49.09 & 0.26 & 0.27 & 1.48 & 12.17 & 8 \\
\hline IR $12112+0305$ & 25 & 6.880 & 2.00 & 7 & 45.80 & 0.35 & 0.20 & 0.30 & 12.24 & - \\
\hline IR 05189-2524*,†,§ & 10 & 6.750 & 2.00 & 6 & 27.37 & 0.40 & 0.65 & 0.56 & 11.93 & 17 \\
\hline UGC $9913^{\S}$ & 35 & 7.340 & 1.80 & 20 & 64.93 & 0.14 & 0.55 & 0.62 & 12.10 & - \\
\hline IR $08572+3915^{*, \S}$ & 7 & 6.590 & 2.00 & 3 & 64.93 & 0.95 & 0.25 & 0.56 & 11.76 & 47 \\
\hline UGC $8696^{\S}$ & 20 & 7.650 & 1.95 & 37 & 56.72 & 0.20 & 0.37 & 2.23 & 11.99 & 11 \\
\hline IR $15250+3609^{\S}$ & 20 & 7.289 & 2.00 & 16 & 53.66 & 0.47 & 0.70 & 0.97 & 11.85 & 20 \\
\hline IR $10565+2448^{\S}$ & 45 & 7.910 & 2.00 & 60 & 47.43 & 0.29 & 0.14 & 1.80 & 11.94 & - \\
\hline UGC $5101^{\S}$ & 7 & 7.440 & 1.95 & 22 & 50.85 & 0.18 & 0.20 & 3.93 & 11.88 & 1 \\
\hline IZW 107 & 30 & 7.770 & 2.00 & 40 & 33.13 & 0.17 & 0.27 & 1.96 & 11.79 & - \\
\hline IR 01364-1042 & 10 & 7.290 & 2.00 & 14 & 116.1 & 0.53 & 0.50 & 1.95 & 11.67 & 11 \\
\hline IR $10173+0828$ & 40 & 7.200 & 2.00 & 14 & 42.22 & 0.27 & 0.41 & 0.41 & 11.68 & - \\
\hline Arp $299^{*}$ & 25 & 7.756 & 2.00 & 43 & 31.75 & 0.17 & 0.70 & 2.28 & 11.68 & - \\
\hline UGC 4881 & 40 & 7.986 & 2.00 & 44 & 49.97 & 0.22 & 0.20 & 2.42 & 11.64 & - \\
\hline CGCG 436-30§ & 15 & 7.500 & 2.00 & 24 & 45.09 & 0.15 & 0.60 & 2.11 & 11.55 & 5 \\
\hline IC 1623 & 25 & 7.635 & 1.80 & 24 & 39.63 & 0.17 & 0.51 & 1.73 & 11.60 & - \\
\hline NGC 1614* & 20 & 7.840 & 2.00 & 50 & 33.13 & 0.17 & 0.30 & 3.46 & 11.48 & 18 \\
\hline UGC $8387^{\S}$ & 20 & 7.720 & 1.95 & 33 & 63.65 & 0.28 & 0.20 & 2.62 & 11.55 & - \\
\hline NGC $7469^{*}$ & 30 & 7.938 & 2.00 & 45 & 33.13 & 0.16 & 0.43 & 2.89 & 11.55 & 10 \\
\hline UGC 2369 & 25 & 7.840 & 2.00 & 36 & 31.70 & 0.12 & 0.67 & 2.77 & 11.55 & - \\
\hline IIIZW 35 & 45 & 7.545 & 1.95 & 35 & 61.20 & 0.18 & 0.55 & 0.78 & 11.50 & - \\
\hline IC 5298* & 20 & 7.900 & 2.00 & 60 & 30.88 & 0.10 & 0.42 & 4.00 & 11.53 & - \\
\hline Arp 148 & 20 & 7.735 & 1.80 & 27 & 75.07 & 0.20 & 0.24 & 2.72 & 11.49 & - \\
\hline NGC $2623^{\S}$ & 35 & 7.726 & 2.00 & 30 & 71.94 & 0.32 & 0.35 & 1.52 & 11.45 & 2 \\
\hline Mrk 331 & 45 & 7.910 & 2.00 & 35 & 53.66 & 0.14 & 0.70 & 1.80 & 11.38 & 5 \\
\hline NGC 34 & 20 & 7.875 & 2.00 & 65 & 42.23 & 0.14 & 0.45 & 3.75 & 11.39 & 4 \\
\hline NGC 5256 & 15 & 7.630 & 1.75 & 15 & 45.09 & 0.30 & 0.60 & 2.84 & 11.41 & 5 \\
\hline UGC 6436 & 30 & 7.990 & 1.90 & 34 & 40.89 & 0.05 & 0.37 & 3.25 & 11.39 & - \\
\hline NGC 6286 & 10 & 7.640 & 1.80 & 23 & 94.25 & 0.50 & 0.47 & 4.36 & 11.20 & 5 \\
\hline Error (\%) & 13.0 & 23.0 & - & 36.0 & 14.0 & 24.0 & - & 12.0 & 6.0 & - \\
\hline
\end{tabular}

instance, the case of IRAS $15250+3609$, with a $J-K=1.16$, typical of a stellar population, but whose SED fitting required an AGN contribution of $\sim 20 \%$ to the total IR luminosity. Another typical AGN diagnostic in the NIR-MIR range is the $25 / 60 \mu \mathrm{m}$ ratio (De Grijp et al. 1985; Sanders et al. 1988). Thus, "warm" galaxies, with $25 / 60 \geq 0.2$ are likely dominated by the AGN, while "cold" galaxies $(25 / 60<0.2)$ are probably dominated by star formation. In Table 1, we mark with a * "warm" galaxies of our sample. However, as a result, we can see in Table 8 that not all the warm galaxies needed an AGN contribution, e.g. Arp 299 $(25 / 60=0.23)$ or IC $5298(25 / 60=0.22)$, and on the contrary, some cold galaxies, IRAS $15250+3609(25 / 60=0.18)$, and UGC $8696(25 / 60=0.10)$, needed AGN contributions to the total IR luminosity higher than $10 \%$.

The FIR to sub-mm spectral range is the least sensitive to the presence of an AGN. In the 40-500 $\mu \mathrm{m}$ range, only two galaxies needed AGN contributions higher than $10 \%$, IRAS 05189-2524, and the completely AGN dominated, IRAS $08572+3915$. Similar results have also been found by Schweitzer et al. (2006) for a sample of 27 Palomar-Green QSOs.

In summary, of the 30 galaxies of our sample, 16 needed an AGN contribution in order to fit the SEDs. Only 1 galaxy, IRAS $08572+3915$, seems to need a dominant AGN contribution $(\sim 47 \%)$ to the total IR luminosity. Seven of these galaxies require an AGN contribution to the IR luminosity $(8-1000 \mu \mathrm{m})$ lower than the estimated error in the IR luminosity ( $\sim 6 \%$, see last row of Col. 10 in Table 5). The NIR to MIR spectral range was well constrained only for the 11 galaxies with a MIR spectrum. Of those, 8 needed an AGN contribution in order to explain their SED+ MIR spectrum, and only UGC 9913, IRAS 10565+2448, 
Table 6. Derived quantities from the best-fit starburst models: $\tau_{\mathrm{ff}}$ is the free-free opacity at $1.4 \mathrm{GHz}, C$ is the fraction, in $\%$, of the total energy of the starburst reprocessed by the cirrus component. $M_{\mathrm{gas}}, M_{\mathrm{D}}, M_{\mathrm{den}}, M_{\star}^{b}$, and $M_{\mathrm{T}}$ are the logarithms of the final gas mass, the dust mass, the dense molecular gas mass, the mass of stars created during the burst, and the total mass of the galaxy calculated as $M_{\mathrm{gas}}+M_{\mathrm{D}}+M_{\star}^{\text {tot }}$. SFR $R_{\mathrm{c}}$ is the star formation rate averaged over the last $10 \mathrm{Myr}$, while $\langle S F R\rangle$ is the SFR averaged over the age of the burst. $T_{\text {th }}$ is the percentage of thermal emission to the $22 \mathrm{GHz}$ flux. The last row gives an estimate of the errors on the parameters as described in Sect. 4.

\begin{tabular}{|c|c|c|c|c|c|c|c|c|c|c|}
\hline Name & $\overline{\tau_{\mathrm{ff}}}$ & $\begin{array}{l}C \\
\%\end{array}$ & $\begin{array}{c}M_{\text {gas }} \\
\left(M_{\odot}\right)\end{array}$ & $\begin{array}{r}M_{\mathrm{D}} \\
\left(M_{\odot}\right)\end{array}$ & $\begin{array}{c}M_{\mathrm{den}} \\
\left(M_{\odot}\right)\end{array}$ & $\begin{array}{r}M_{\star}^{b} \\
\left(M_{\odot}\right)\end{array}$ & $\begin{array}{r}M_{\mathrm{T}} \\
\left(M_{\odot}\right)\end{array}$ & $\begin{array}{c}\langle S F R\rangle \\
\left(\frac{M_{\odot}}{\mathrm{yr}}\right)\end{array}$ & $\begin{array}{l}S F R_{\mathrm{c}} \\
\left(\frac{M_{\odot}}{\mathrm{yr}}\right)\end{array}$ & $\begin{array}{r}T_{\text {th }} \\
\%\end{array}$ \\
\hline UGC 8058 & 0.03 & 3.0 & 10.26 & 8.26 & 10.08 & 10.40 & 11.41 & 681.5 & 435.8 & 20.7 \\
\hline IR $14348-1447$ & $2 e-3$ & 12.5 & 10.44 & 8.44 & 9.86 & 9.86 & 10.95 & 492.3 & 336.3 & 20.7 \\
\hline IR $12112+0305$ & 0.28 & 13.8 & 10.44 & 8.44 & 9.98 & 9.59 & 11.07 & 508.3 & 502.9 & 50.4 \\
\hline IR $05189-2524$ & 0.02 & 5.0 & 10.02 & 8.02 & 9.62 & 9.18 & 10.51 & 260.8 & 225.8 & 66.4 \\
\hline UGC 9913 & 0.56 & 7.0 & 10.65 & 8.65 & 9.79 & 9.80 & 11.10 & 288.3 & 235.1 & 33.2 \\
\hline IR $08572+3915$ & 0.58 & 1.9 & 9.16 & 7.16 & 9.14 & 8.90 & 10.13 & 205.5 & 129.8 & 78.9 \\
\hline UGC 8696 & 0.01 & 7.7 & 10.41 & 8.41 & 9.71 & 10.25 & 11.19 & 400.8 & 137.8 & 19.3 \\
\hline IR $15250+3609$ & 1.08 & 2.0 & 9.77 & 7.77 & 9.44 & 9.61 & 10.67 & 209.8 & 154.5 & 29.6 \\
\hline IR $10565+2448$ & $7 e-3$ & 25.0 & 10.29 & 8.29 & 9.75 & 10.36 & 11.27 & 281.5 & 116.3 & 25.4 \\
\hline UGC 5101 & 0.13 & 20.9 & 10.55 & 8.55 & 9.81 & 10.10 & 11.08 & 454.8 & 71.5 & 4.8 \\
\hline IZW 107 & 0.03 & 13.0 & 10.24 & 8.24 & 9.50 & 10.12 & 11.06 & 225.8 & 87.2 & 22.5 \\
\hline IR 01364-1042 & 0.76 & 3.7 & 9.66 & 7.66 & 9.38 & 9.55 & 10.50 & 180.7 & 91.7 & 26.0 \\
\hline IR $10173+0828$ & 0.67 & 7.0 & 10.06 & 8.06 & 9.36 & 9.28 & 10.66 & 120.3 & 109.7 & 41.7 \\
\hline Arp 299 & 0.03 & 5.3 & 10.19 & 8.19 & 9.41 & 10.07 & 11.00 & 207.4 & 67.3 & 15.6 \\
\hline UGC 4881 & $1 e-3$ & 21.0 & 10.05 & 8.05 & 9.39 & 10.25 & 11.12 & 184.0 & 53.8 & 24.7 \\
\hline CGCG 436-30 & 0.39 & 6.4 & 10.02 & 8.02 & 9.20 & 9.67 & 10.65 & 146.5 & 58.0 & 23.1 \\
\hline IC 1623 & $4 e-4$ & 10.8 & 10.18 & 8.18 & 9.41 & 9.78 & 10.82 & 139.2 & 65.5 & 19.4 \\
\hline NGC 1614 & 0.24 & 11.6 & 9.89 & 7.89 & 9.12 & 10.09 & 10.95 & 179.6 & 29.4 & 18.1 \\
\hline UGC 8387 & 0.31 & 17.1 & 10.07 & 8.07 & 9.52 & 9.93 & 10.84 & 160.9 & 43.5 & 14.8 \\
\hline NGC 7469 & 0.02 & 14.3 & 10.04 & 8.04 & 9.24 & 10.20 & 11.06 & 181.2 & 40.8 & 20.1 \\
\hline UGC 2369 & 0.13 & 11.4 & 10.28 & 8.28 & 9.36 & 10.13 & 11.04 & 194.1 & 47.3 & 23.7 \\
\hline IIIZW 35 & 0.64 & 2.3 & 10.07 & 8.07 & 9.33 & 9.46 & 10.64 & 81.9 & 60.0 & 29.1 \\
\hline IC 5298 & 0.03 & 12.8 & 10.28 & 8.28 & 9.28 & 10.21 & 11.06 & 206.6 & 25.5 & 26.8 \\
\hline Arp 148 & 0.32 & 21.6 & 10.06 & 8.06 & 9.36 & 9.90 & 10.82 & 146.2 & 37.3 & 16.8 \\
\hline NGC 2623 & 0.42 & 8.7 & 9.77 & 7.77 & 9.28 & 9.70 & 10.66 & 95.3 & 47.0 & 23.1 \\
\hline Mrk 331 & 0.17 & 9.9 & 10.08 & 8.08 & 9.23 & 9.87 & 10.82 & 91.3 & 37.7 & 31.8 \\
\hline NGC 34 & 0.01 & 7.8 & 9.93 & 7.93 & 9.08 & 10.05 & 10.91 & 150.7 & 21.0 & 18.3 \\
\hline NGC 5256 & 0.01 & 15.0 & 9.77 & 7.77 & 9.25 & 9.85 & 10.73 & 164.7 & 40.7 & 11.0 \\
\hline UGC 6436 & 0.19 & 27.1 & 10.36 & 8.36 & 9.07 & 10.12 & 11.04 & 135.3 & 25.0 & 22.2 \\
\hline NGC 6286 & $5 e-3$ & 13.6 & 9.65 & 7.65 & 9.35 & 9.77 & 10.59 & 136.1 & 14.0 & 5.2 \\
\hline Errors (\%) & - & - & 56.0 & 56.0 & 17.0 & 22.0 & 8.0 & 3.0 & 7.0 & - \\
\hline
\end{tabular}

and UGC 8387 could be well modelled with pure starburst models.

\section{Discussion}

One of the main results emerging from our investigation is that in all but one galaxy, IRAS $08572+3915$, the starburst dominates the bolometric ( IR) emission of the source, with a fractional contribution that is always larger than $80 \%$. About half of the sample can actually be fitted by a pure starburst.

We thus begin the discussion by looking into the main features of the SB components of (U)LIRGs and their possible differences to normal starburst galaxies.

\subsection{The starburst component}

All the pure-starburst (U)LIRGs in our sample have $e$-folding times ranging between 20 and $50 \mathrm{Myr}$, while some shorter $e$ folding times are obtained for the mixed (AGN + SB) subsample. In Fig. 3, we show the distribution of $t_{\mathrm{b}}$ for pure SB and mixed (U)LIRGs. A $K-S$ test for the two distributions indicates a high probability that the two distributions are different. This would indicate that (U)LIRGs with an AGN are characterized by a more rapidly declining SFR than pure starburst (U)LIRGs.
An exciting interpretation of this finding is that the SFR itself suffers negative feedback from the AGN.

On the contrary, no significant differences are found in the other parameters, i.e. dust opacities, escape times, etc.

The ranges of ages varies from $3.9 \times 10^{6} \mathrm{yr}$ for IRAS $08572+3915$ to $9.8 \times 10^{7}$ yr for UGC 6436 , with a small trend for older bursts in pure SB objects.

More interesting is the comparison of the MC optical depth, $\tau_{1}$, and the degree of obscuration, measured by the quantity age $_{\mathrm{b}} / t_{\mathrm{esc}}$, between (U)LIRGs and normal starburst galaxies. Both parameters indicate that (U)LIRGs constitute a population of very dust enshrouded objects compared to normal starburst galaxies. In Fig. 4, we show the distributions of $\tau_{1}$ (left panel) and $\mathrm{age}_{\mathrm{b}} / t_{\mathrm{esc}}$ (right panel) for our sample of (U)LIRGs (solid line) and the sample of normal starburst from Vega et al. (2005) (dashed line). Both histograms indicate that (U)LIRGs and starburst are two very distinct populations and that, consequently, the infrared properties of (U)LIRGs cannot be obtained from those of starburst galaxies just by re-scaling them to higher star formation rates.

The values of the SB evolutionary parameter, $E_{\mathrm{SB}}$, show that many LIRGs and ULIRGs (15/30) are found in the peak phase of the starburst phenomenon $\left(0.20 \leqslant E_{\mathrm{SB}}<2\right)$. None of our sample galaxies is in the early phase $\left(E_{\mathrm{SB}}<0.2\right)$. This is expected both because this phase is of relatively short duration and because 
Table 7. AGN fitted parameters. See text for details.

\begin{tabular}{lccccccr|r}
\hline \hline Name & $\begin{array}{c}\Theta \\
(\mathrm{deg})\end{array}$ & $r_{\mathrm{m}} / r_{0}$ & $\beta$ & $\begin{array}{c}\Theta_{\mathrm{h}} \\
(\mathrm{deg})\end{array}$ & $\alpha$ & $\begin{array}{c}a_{\max } \\
(\mu \mathrm{m})\end{array}$ & $\tau_{\mathrm{e}, 9.7}$ & $\begin{array}{r}r_{\mathrm{m}}^{(a)} \\
(\mathrm{pc})\end{array}$ \\
\hline UGC 8058 & 40 & 1000 & 0.5 & 20 & 6.0 & 0.3 & 10.0 & 68.98 \\
IR 14348-1447 & 46 & 3000 & 0.5 & 40 & 0.0 & 0.3 & 3.0 & 99.00 \\
IR 05189-2524 & 41 & 1000 & 0.0 & 40 & 0.0 & 0.5 & 10.0 & 47.29 \\
IR 08572+3915 & 77 & 1000 & 1.0 & 40 & 6.0 & 0.3 & 10.0 & 62.48 \\
UGC 8696 & 74 & 1000 & 0.5 & 40 & 6.0 & 1.0 & 10.0 & 29.01 \\
IR 15250+3609 & 90 & 300 & 1.0 & 40 & 0.0 & 0.3 & 10.0 & 10.38 \\
UGC 5101 & 10 & 3000 & 1.0 & 40 & 3.0 & 0.3 & 3.0 & 56.44 \\
IR 01364-1042 & 62 & 300 & 0.0 & 20 & 3.0 & 1.0 & 10.0 & 6.01 \\
CGCG 436-30 & 40 & 1000 & 0.0 & 40 & 0.0 & 0.3 & 10.0 & 18.08 \\
NGC 1614 & 74 & 1000 & 1.0 & 40 & 6.0 & 1.0 & 10.0 & 22.24 \\
NGC 7469 & 21 & 1000 & 0.0 & 20 & 0.0 & 1.3 & 3.0 & 19.67 \\
NGC 2623 & 22 & 3000 & 0.5 & 20 & 0.0 & 0.3 & 3.0 & 22.44 \\
Mrk 331 & 48 & 3000 & 0.5 & 40 & 6.0 & 0.3 & 10.0 & 32.05 \\
NGC 34 & 60 & 1000 & 0.5 & 40 & 0.0 & 0.3 & 1.0 & 9.79 \\
NGC 5256 & 39 & 3000 & 1.0 & 40 & 0.0 & 0.3 & 1.0 & 67.58 \\
NGC 6286 & 40 & 3000 & 1.0 & 20 & 6.0 & 0.3 & 10.0 & 32.57 \\
\hline
\end{tabular}

(a) Maximum radius of the torus model. It was calculated from the equation given in Sect. 3 in Granato \& Danese (1994) for $r_{0}$ and the value of the parameter $r_{\mathrm{m}} / r_{0}$ given in Col. (3) of this table.

the number of newly born stars in the early phase is insufficient to power the infrared luminosity. Fourteen (U)LIRGs are in an older phase $\left(2<E_{\mathrm{SB}}<4\right)$, and only one, the least IR luminous NGC 6286, is found in the post-starburst phase. The starburst phase defined by the IR colours (an observational quantity) is in perfect agreement with that defined by the $E_{\mathrm{SB}}$ parameter (a theoretical quantity), see Fig. 5. In the same figure we notice that when the estimated AGN contribution is subtracted from the fluxes in the IR bands, (U)LIRGs with AGN show the same trend as pure starburst (U)LIRGs (open circles connected to solid circles), indicating that our determination of the AGN contributions is robust.

Another important quantity derived from our fits is the mass of dust. Dunne et al. (2000) have published dust mass measurements for their SLUGS sample, which includes some of our objects. In general, there is a very good agreement between their determinations from $850 \mu \mathrm{m}$ observations and ours. However, for three galaxies, IRAS $08572+3915$, IRAS $15250+3909$, and NGC 1614, their estimates are considerably higher than ours, reaching one order of magnitude in the case of IRAS $08572+3915$. The likely origin of the discrepancy is that the Dunne et al. (2000) determinations are based on the assumption that the dust emitting in the sub-mm range is cold $(T \sim 20 \mathrm{~K})$, while GRASIL accounts for a distribution of dust temperatures within the ISM. In the above three galaxies we can see from the fits that the sub-mm spectral range is still dominated by a warm MC component which thus requires a significantly lower dust mass. We believe that our estimate is more correct, since it is based on the fit to the whole MIR-FIR-sub-mm spectral range.

The total baryonic mass derived for our sample of (U)LIRGs is also in good agreement with dynamical mass estimates (e.g. Genzel et al. 2001; Colina et al. 2005; Dasyra et al. 2006). Moreover, our estimates of the total gas mass are in agreement with those derived from available observations in $\mathrm{HI}$ and in $\mathrm{CO}$, see for instance Cols. 7 and 8 in Table 4 by Dunne et al. (2000), provided that a reduced conversion factor (X-factor) between $\mathrm{CO}$ luminosity and mass of molecular gas is used (Solomon et al. 1997).

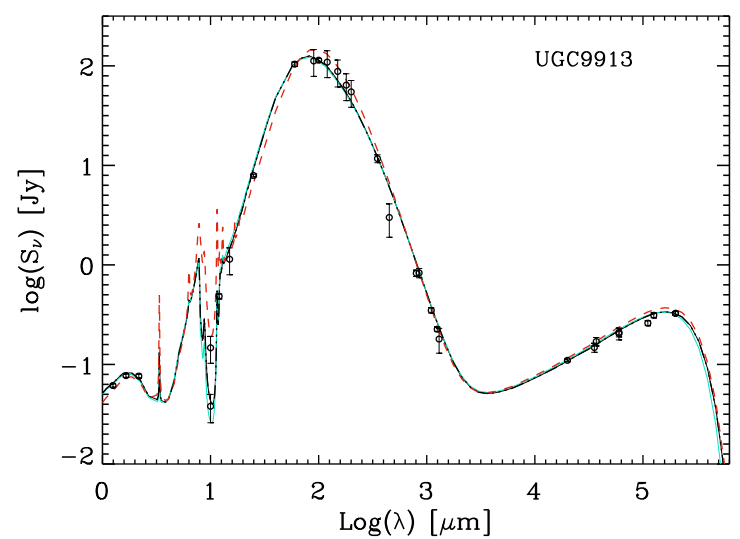

Fig. 2. Fits to the UGC 9913 observed and simulated SEDs performed to estimate the errors in the different model parameters and derived quantities. The open circles represent the observational data. The three dotsdashed line is the best fit to the data while the thin solid line and the dotted line represent the best fits to two simulated data-sets drawn randomly from the observed values \pm a full standard deviation.

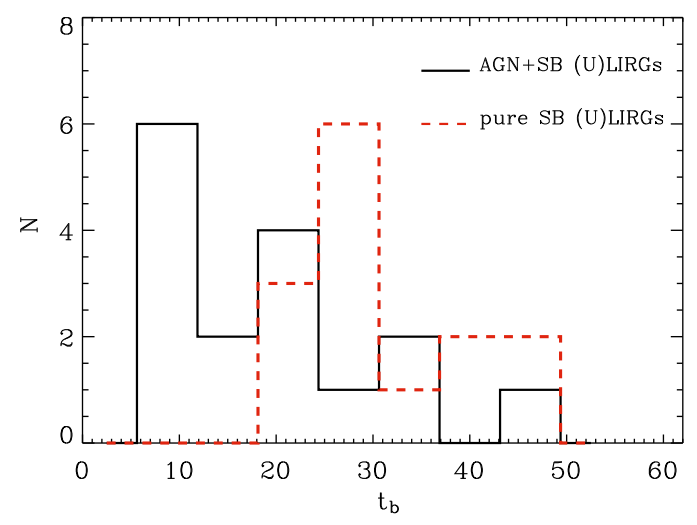

Fig. 3. Distributions of the $e$-folding times of the burst for pure $\mathrm{SB}$ (U)LIRGs (dashed line) and for mixed (AGN+SB) (U)LIRGs (solid line).

Finally, in Fig. 7 we plot the current SFR, $S F R_{\mathrm{c}}$, versus the AGN corrected IR luminosity of the best-fit models, $L_{\mathrm{IR}}^{\mathrm{SB}}$. The solid line represents the best linear fit to the data, i.e. $S F R=1.82 \times 10^{-10} L_{\mathrm{IR}}\left(M_{\odot} L_{\odot}^{-1}\right)$. For comparison we also plot the $S F R / L_{\mathrm{IR}}$ calibration of Kennicutt (1998) (dashed line). The two calibrations coincide within the accuracy of the models (Kennicutt 1998).

\subsection{Spitzer spectra}

In our starburst models the MIR spectral domain is largely dominated by the MC component. However, in (U)LIRGs this spectral region may be significantly affected by the presence of an AGN. The interpretation of the broad-band MIR fluxes may be affected by a degeneracy between the optical depth of the MCs and the contribution of the putative AGN. Indeed observed warm MIR colours may be almost equally well reproduced by increasing the MC optical depth and, at the same time, increasing the AGN contribution (see e.g. IRAS 08572+3915 in Fig. 1 of Prouton et al. 2004). However, an increase of the MC optical depth has the effect of strengthening the $9.7 \mu \mathrm{m}$ silicate absorption feature. Thus, a possible way to break this degeneracy is to complement the broad band fluxes with the 5-35 $\mu \mathrm{m}$ Spitzer IRS spectrum which carries a great deal of additional information. 
Table 8. Total (SB + AGN) luminosity from the SED models in different spectral windows and the corresponding AGN contribution. Galaxies marked with an * have warm IR colors, $f_{25} / f_{60}>0.2$. Galaxies marked with a ${ }^{\ddagger}$ have NIR colours $J-K>1.7$, and those marked with a ${ }^{\S}$ have available Spitzer IRS spectra.

\begin{tabular}{|c|c|c|c|c|c|c|c|}
\hline Name & $\begin{array}{c}L_{1-5} / \mathrm{AGN} \\
\left(L_{\odot} / \%\right) \\
\end{array}$ & $\begin{array}{c}L_{3-30} / \mathrm{AGN} \\
\left(L_{\odot} / \%\right) \\
\end{array}$ & $\begin{array}{c}L_{8-1000} / \mathrm{AGN} \\
\left(L_{\odot} / \%\right) \\
\end{array}$ & $\begin{array}{c}L_{1-1000} / \mathrm{AGN} \\
\left(L_{\odot} / \%\right) \\
\end{array}$ & $\begin{array}{c}L_{40-500 / \mathrm{AGN}} \\
\left(L_{\odot} / \%\right)\end{array}$ & $\begin{array}{c}L_{3-1100} / \mathrm{AGN} \\
\left(L_{\odot} / \%\right) \\
\end{array}$ & $\begin{array}{c}L_{\text {bol }} / \mathrm{AGN} \\
\left(L_{\odot} / \%\right) \\
\end{array}$ \\
\hline UGC $8058^{*,+, \S}$ & $11.64 / 71$ & $11.66 / 42$ & $12.42 / 16$ & $12.51 / 24$ & $12.18 / 7$ & $12.48 / 21$ & $12.51 / 24$ \\
\hline IR $14348-1447^{\ddagger}$ & $10.75 / 56$ & $11.48 / 27$ & $12.21 / 8$ & $12.23 / 10$ & $12.07 / 6$ & $12.23 / 10$ & $12.23 / 10$ \\
\hline IR 05189-2524, $4^{*,+,}$ & $11.01 / 74$ & $11.60 / 30$ & $12.01 / 17$ & $12.07 / 23$ & $11.75 / 13$ & $12.05 / 20$ & $12.20 / 23$ \\
\hline IR $08572+3915^{*,+}, 8$ & $10.88 / 91$ & $11.64 / 81$ & $12.04 / 47$ & $12.09 / 52$ & $11.77 / 30$ & $12.09 / 52$ & $12.09 / 52$ \\
\hline UGC $8696^{\S}$ & $10.68 / 14$ & $11.29 / 29$ & $12.05 / 11$ & $12.07 / 12$ & $11.90 / 6$ & $12.06 / 12$ & $12.08 / 12$ \\
\hline IR $15250+3609^{\S}$ & $10.45 / 30$ & $11.37 / 49$ & $11.94 / 20$ & $11.97 / 21$ & $11.73 / 8$ & $11.96 / 21$ & $11.97 / 21$ \\
\hline UGC $5101^{\ddagger, \S}$ & $10.71 / 53$ & $11.06 / 12$ & $11.89 / 1$ & $11.92 / 4$ & $11.79 / 0$ & $11.90 / 2$ & $11.97 / 6$ \\
\hline IR 01364-1042 & $10.13 / 16$ & $10.85 / 60$ & $11.73 / 11$ & $11.74 / 12$ & $11.61 / 3$ & $11.74 / 12$ & $11.75 / 12$ \\
\hline CGCG $436-30^{\S}$ & $10.38 / 42$ & $10.92 / 14$ & $11.57 / 5$ & $11.61 / 7$ & $11.41 / 3$ & $11.59 / 6$ & $11.71 / 10$ \\
\hline NGC $1614^{*}$ & $10.55 / 18$ & $11.11 / 37$ & $11.58 / 18$ & $11.63 / 19$ & $11.35 / 8$ & $11.61 / 20$ & $11.65 / 18$ \\
\hline NGC $7469^{*}$ & $10.76 / 20$ & $11.06 / 25$ & $11.55 / 10$ & $11.63 / 12$ & $11.36 / 4$ & $11.59 / 12$ & $11.70 / 13$ \\
\hline NGC $2623^{\S}$ & $10.25 / 15$ & $10.56 / 12$ & $11.46 / 2$ & $11.50 / 3$ & $11.37 / 1$ & $11.48 / 3$ & $11.51 / 3$ \\
\hline MRK 331 & $10.47 / 20$ & $10.71 / 20$ & $11.40 / 5$ & $11.46 / 6$ & $11.27 / 3$ & $11.43 / 6$ & $11.48 / 6$ \\
\hline NGC 34 & $10.47 / 17$ & $10.79 / 17$ & $11.40 / 4$ & $11.46 / 5$ & $11.24 / 1$ & $11.43 / 5$ & $11.48 / 5$ \\
\hline NGC 5256 & $10.79 / 45$ & $10.91 / 25$ & $11.43 / 5$ & $11.54 / 12$ & $11.28 / 2$ & $11.47 / 8$ & $11.71 / 16$ \\
\hline NGC 6286 & $10.50 / 32$ & $10.46 / 41$ & $11.22 / 5$ & $11.31 / 9$ & $11.15 / 1$ & $11.26 / 8$ & $11.33 / 9$ \\
\hline
\end{tabular}

Table 9. Total (SB + AGN) flux from the SED models in different IR spectral bands and the corresponding AGN contribution. Galaxies marked with an ${ }^{*}$ have warm IR colors, $f_{25} / f_{60}>0.2$. Galaxies marked with a ${ }^{*}$ have NIR colours $J-K>1.7$, and those marked with a ${ }^{\S}$ have available Spitzer IRS spectra. For the latter galaxies the lower row provides fluxes calculated from the Spitzer spectra in the IRAC 3 and IRAC 4 and MIPS $24 \mu \mathrm{m}$ bands. In the IRAC bands, differences to models in the upper row are mainly attributable to effects of molecular absorption bands.

\begin{tabular}{|c|c|c|c|c|c|c|c|c|c|c|}
\hline Name & $\begin{array}{c}H \\
1.23 \mu \mathrm{m} \\
(\mathrm{Jy} / \%) \\
\end{array}$ & $\begin{array}{c}J \\
1.66 \mu \mathrm{m} \\
(\mathrm{Jy} / \%) \\
\end{array}$ & $\begin{array}{c}K \\
2.16 \mu \mathrm{m} \\
(\mathrm{Jy} / \%) \\
\end{array}$ & $\begin{array}{c}\text { IRAC } 1 \\
3.55 \mu \mathrm{m} \\
(\mathrm{Jy} / \%) \\
\end{array}$ & $\begin{array}{c}\text { IRAC } 2 \\
4.5 \mu \mathrm{m} \\
(\mathrm{Jy} / \%) \\
\end{array}$ & $\begin{array}{c}\text { IRAC 3 } \\
5.73 \mu \mathrm{m} \\
(\mathrm{Jy} / \%)\end{array}$ & $\begin{array}{c}\text { IRAC } 4 \\
8 \mu \mathrm{m} \\
(\mathrm{Jy} / \%) \\
\end{array}$ & $\begin{array}{c}\text { MIPS 1 } \\
24 \mu \mathrm{m} \\
(\mathrm{Jy} / \%) \\
\end{array}$ & $\begin{array}{c}\text { MIPS } 2 \\
70 \mu \mathrm{m} \\
(\mathrm{Jy} / \%) \\
\end{array}$ & $\begin{array}{l}\text { MIPS 3 } \\
160 \mu \mathrm{m} \\
(\mathrm{Jy} / \%) \\
\end{array}$ \\
\hline UGC $8058^{*, \hbar, \S}$ & $0.06 / 24$ & $0.11 / 52$ & $0.21 / 76$ & $0.38 / 90$ & $0.48 / 82$ & $\begin{array}{l}0.70 / 62 \\
0.70 / 62\end{array}$ & $\begin{array}{l}1.16 / 43 \\
0.85 / 64\end{array}$ & $\begin{array}{l}6.75 / 27 \\
6.70 / 27\end{array}$ & $34.91 / 6$ & $14.82 / 3$ \\
\hline IR $14348-1447^{\ddagger}$ & $1.7 \mathrm{E}-3 / 5$ & $5 E-3 / 11$ & $3.2 \mathrm{E}-3 / 34$ & $0.01 / 83$ & $0.02 / 79$ & $0.04 / 55$ & $0.08 / 31$ & $0.57 / 18$ & $6.92 / 6$ & $4.22 / 3$ \\
\hline IR 05 & $02 / 77$ & $0.03 / 83$ & $0.04 / 90$ & $0.07 / 88$ & $0.11 / 65$ & $\begin{array}{l}0.21 / 41 \\
0.15 / 57\end{array}$ & $\begin{array}{l}0.43 / 27 \\
0.28 / 41\end{array}$ & $\begin{array}{l}2.62 / 24 \\
2.78 / 23\end{array}$ & $12.94 / 12$ & $5.86 / 6$ \\
\hline IR $08572+3915^{*,+,}, \S$ & $1.7 \mathrm{E}-3 / 26$ & $2.8 \mathrm{E}-3 / 44$ & $3.7 \mathrm{E}-3 / 63$ & $0.02 / 95$ & $0.07 / 97$ & $\begin{array}{l}0.17 / 95 \\
0.19 / 85\end{array}$ & $\begin{array}{l}0.26 / 92 \\
0.34 / 71\end{array}$ & $\begin{array}{l}1.67 / 72 \\
1.54 / 78\end{array}$ & $6.78 / 27$ & $1.83 / 18$ \\
\hline UGC $8696^{\S}$ & $0.02 / 2$ & $0.03 / 4$ & $0.03 / 11$ & $0.02 / 34$ & $0.03 / 38$ & $\begin{array}{l}0.08 / 27 \\
0.07 / 27\end{array}$ & $\begin{array}{l}0.20 / 22 \\
0.16 / 27\end{array}$ & $\begin{array}{l}2.40 / 31 \\
2.10 / 35\end{array}$ & $22.82 / 6$ & $12.07 / 2$ \\
\hline IR $15250+3609^{\S}$ & $3 \mathrm{E}-3 / 0$ & $5.5 \mathrm{E}-3 / 0$ & $4.9 \mathrm{E}-3 / 0$ & $3.7 \mathrm{E}-3 / 17$ & $0.01 / 59$ & $\begin{array}{l}0.05 / 64 \\
0.04 / 80\end{array}$ & $\begin{array}{l}0.12 / 55 \\
0.10 / 66\end{array}$ & $\begin{array}{l}1.27 / 47 \\
1.25 / 48\end{array}$ & $7.23 / 6$ & $2.70 / 2$ \\
\hline UGC 510 & $0.02 / 38$ & $0.03 / 51$ & $0.03 / 63$ & $0.03 / 64$ & $0.03 / 58$ & $\begin{array}{l}0.07 / 26 \\
0.09 / 20\end{array}$ & $\begin{array}{c}0.20 / 8 \\
0.14 / 11\end{array}$ & $\begin{array}{l}0.96 / 4 \\
0.93 / 4\end{array}$ & $15.51 / 0$ & $13.52 / 0$ \\
\hline IR 01 & $4.0 \mathrm{E}-3 / 0$ & $5.2 \mathrm{E}-3 / 1$ & $4.9 \mathrm{E}-3 / 5$ & $4.6 \mathrm{E}-3 / 31$ & $4.7 E-3 / 62$ & $0.01 / 65$ & $0.04 / 48$ & $0.50 / 60$ & $7.68 / 2$ & $3.26 / 0$ \\
\hline CGCG & $0.01 / 26$ & $0.02 / 34$ & $0.02 / 48$ & $0.02 / 64$ & $0.03 / 53$ & $\begin{array}{l}0.07 / 27 \\
0.04 / 47\end{array}$ & $\begin{array}{l}0.15 / 15 \\
0.12 / 19\end{array}$ & $\begin{array}{l}1.31 / 8 \\
1.31 / 8\end{array}$ & $10.69 / 3$ & $5.36 / 1$ \\
\hline NGC & $0.09 / 3$ & $1 / 6$ & 0 & $0.11 / 37$ & 0.18 & $0.44 / 35$ & $1.01 / 33$ & $7.44 / 37$ & $35.28 / 7$ & $18.72 / 2$ \\
\hline NGC & $0.15 / 6$ & $0.19 / 10$ & $0.18 / 19$ & $0.19 / 42$ & $0.23 / 47$ & $0.45 / 32$ & $1.09 / 19$ & $5.40 / 23$ & $34.43 / 3$ & $22.17 / 1$ \\
\hline NGC 2 & $0.04 / 2$ & $0.05 / 5$ & $0.05 / 13$ & $0.04 / 37$ & $0.04 / 48$ & $\begin{array}{l}0.09 / 27 \\
0.04 / 61\end{array}$ & $\begin{array}{l}0.21 / 12 \\
0.14 / 18\end{array}$ & $\begin{array}{l}1.73 / 7 \\
1.70 / 7\end{array}$ & $29.29 / 1$ & $15.04 / 1$ \\
\hline $\mathrm{N}$ & $0.06 / 1$ & $07 / 5$ & & (1) & 0.09 & 0.1 & & $2.05 / 11$ & $22.51 / 2$ & $14.04 / 1$ \\
\hline NGC & $0.05 / 1$ & $0.07 / 3$ & $06 / 12$ & $0.06 / 47$ & $0.08 / 49$ & $0.15 / 32$ & $0.29 / 22$ & $2.40 / 8$ & $18.13 / 1$ & $9.74 / 0$ \\
\hline NGC 5 & $0.05 / 24$ & $0.07 / 34$ & $0.08 / 49$ & $0.08 / 64$ & $0.08 / 70$ & $0.12 / 43$ & $0.34 / 16$ & $0.98 / 12$ & $9.76 / 1$ & $7.66 / 0$ \\
\hline NGC 6286 & $0.06 / 5$ & $0.08 / 13$ & $0.09 / 32$ & $0.10 / 61$ & $0.09 / 77$ & $0.13 / 58$ & $0.33 / 23$ & $0.61 / 29$ & $16.93 / 1$ & $15.48 / 0$ \\
\hline
\end{tabular}

We thus searched for Spitzer IRS spectra of our ULIRG sample in the SSC archive and found them for 11 galaxies. For these galaxies the fitting procedure also includes the Spitzer spectra shown in Fig. 1, together with the corresponding models upwardly displaced by one dex, as solid thick and solid thin lines, respectively.

The largest effect, after accounting for the analysis of the Spitzer spectra, was found for IRAS $15250+3609$. Figure 6 illustrates how the inclusion of the IRS spectrum changes our spectral classification of IRAS $15250+3609$. The left panel shows the best fit to the broad band SED alone. The 20 continuum passbands are very well fitted by a pure SB model with an $e$-folding time of $25 \mathrm{Myr}$, an age of $10^{7} \mathrm{yr}, \tau_{1}$ of 29 and a large current star forming rate, $S F R_{\mathrm{c}} \sim 246 M_{\odot} / \mathrm{yr}$. However, the IRS spectrum of IRAS $15250+3609$ shows the presence of a deep silicate absorption feature at $9.7 \mu \mathrm{m}$ (right panel). With a starburst alone, it is not possible to reproduce the large optical depth required by this silicate absorption feature and the emission in the MIRFIR continuum, at the same time. Indeed, the fit shown in the right panel includes an obscured AGN (torus inclination $\sim 90^{\circ}$ ) 

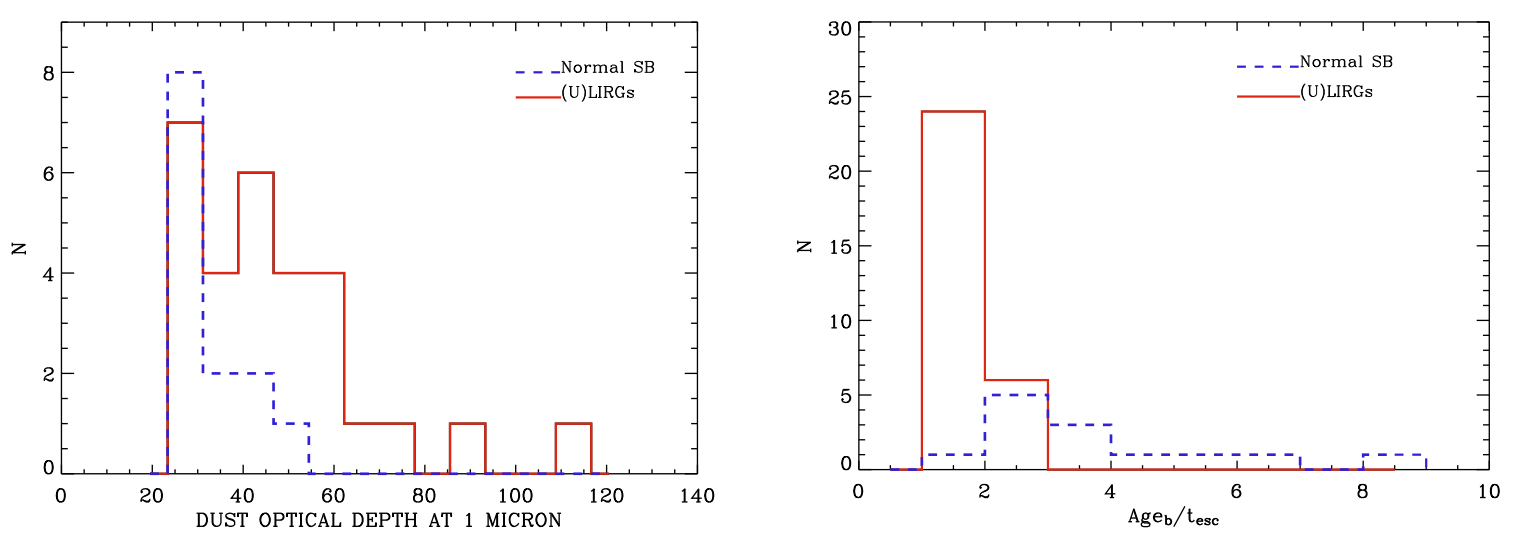

Fig. 4. Left panel: histogram of the distributions of $\tau_{1}$ for our sample of (U)LIRGs (solid line), and a sample of normal starburst galaxies (dashed line). Right panel: histogram of the distributions of the ratio $\mathrm{Age}_{\mathrm{b}} / t_{\mathrm{esc}}$ for the (U)LIRGs (solid line), and the starburst galaxies (dashed line). This ratio gives the degree of obscuration of the newly born stars. See text for details.

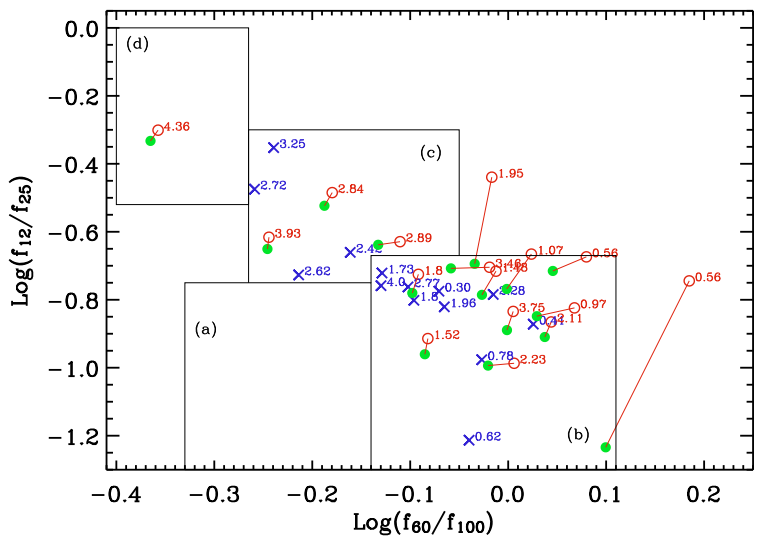

Fig. 5. IRAS colour-colour diagram for our sample (U)LIRGs. Crosses denote the pure starburst galaxies, while open circles denote the galaxies with AGN. The filled circles denote the mixed (SB + AGN) galaxies but with the IR colours corrected for the AGN contributions. The numbers correspond to the values of the parameter $E_{\mathrm{SB}}$. The four closed regions highlight the four evolutionary phases of our starburst model: (a) early phase, (b) peak phase, (c) the old starburst phase, and (d) the post-starburst phase.

contributing $\sim 20 \%$ of the IR $(8-1000 \mu \mathrm{m})$ luminosity, and about $50 \%$ to the MIR luminosity. We notice that, although the contribution of the AGN is only $20 \%$ of the IR luminosity, other quantities have changed by a significant factor. The optical depth of the MCs has almost doubled, and now it fits better in the range of (U)LIRGs. The current SFR has decreased from $\sim 246 M_{\odot} / \mathrm{yr}$ to $\sim 154 M_{\odot} /$ yr while the model still fits the radio data very well. This happens because the evolutionary phase becomes more advanced ( $E_{\mathrm{SB}} \sim 1$ instead of 0.4$)$ and what actually fuels the radio flux is the SNII rate, which probes a time interval five times larger than the $10 \mathrm{Myr}$ adopted for the current SFR. The common belief (e.g. Elvis et al. 1994) is that the presence of the AGN eventually dilutes the $9.7 \mu \mathrm{m}$ feature. IRAS $15250+3609$ actually shows the opposite, since the $9.7 \mu \mathrm{m}$ feature is intrinsically less pronounced in the less opaque MCs of the pure starburst model.

In the other 10 galaxies, the inclusion of the IRS spectrum did not change our classification, although it allowed a more accurate estimate of the SB parameters and of the AGN contribution.

In summary, of the subsample with IRS spectra, three galaxies, UGC 9913, IRAS 10565+2448 and UGC 8387, were still well fitted by pure SB models. Seven galaxies, UGC 8058, UGC 8696, UGC 5101, NGC 2623 CGCG 436-30, IRAS 05189-2524 and IRAS 08572+3915 were fitted with almost the same SB and AGN components. In IRAS 15250+3609 the IRS spectra revealed the presence of an almost entirely obscured AGN contributing 20\% of the IR luminosity. This is where the combined analysis of the IRS spectra and panchromatic SED is seen at its most powerful.

\subsection{Star formation versus AGN}

Sixteen galaxies $(53 \%)$ of our sample of 30 (U)LIRGs require an AGN contribution in order to fit their SEDs. However, only 9 (30\%) need a contribution to the IR luminosity which is higher than $10 \%$. In most cases it is lower than $20 \%$. Only one galaxy, IRAS $08572+3915$, seems to be AGN dominated $(\gtrsim 50 \%$, see the discussion below). In 2/30 (6\%) of objects, the AGN contribution $(8-1000 \mu \mathrm{m})$ is less than $2 \%$. In these objects the AGN contribution is more important at NIR wavelengths, where it may reach $15 \%$ or more, as for UGC $5101(\sim 50 \%)$, while the AGN contribution to the IR (8-1000) luminosity is $\sim 1 \%$. However, UGC 5101 shows an evident power law NIR SED and $[\mathrm{NeV}] 15 \mu \mathrm{m}$ emission in the Spitzer IRS spectrum (Armus et al. 2004). In the remaining 5/30 (17\%) the AGN contribution $(8-1000)$ is of the order of $5 \%$.

For those objects that have been observed with Spitzer, we find that our estimates of the AGN contribution agree very well with those derived by Armus et al. (2007) using the line ratios $\mathrm{Ne}[\mathrm{V}] / \mathrm{Ne}[\mathrm{II}]$ or $\mathrm{O}[\mathrm{IV}] / \mathrm{Ne}[\mathrm{II}]$, although many of their values are quoted as upper limits. We find a different result for two galaxies, IRAS 14348-1447 and IRAS $15250+3609$. The first was classified as a pure SB by Armus et al. (2007), based on the strength of the PAH features. However, we require an AGN contribution of about $10 \%$ to the IR luminosity, in order to match its red NIR colour $(J-K=1.7)$. In the case of IRAS $15250+3609$, Armus et al. find that the power source remains buried even in the MIR, while our analysis of the NIR to radio SED (including the IRS spectrum) requires a buried AGN contributing about $20 \%$ to the IR luminosity.

Armus et al. (2007) have estimated the AGN fractions also with other methods, using either the equivalent width of the $6.2 \mu \mathrm{m}$ PAH feature, the mid-infrared spectral slope, or the ratio between the total PAH flux and the IR flux. These methods provide AGN fractions that are, in general, very much higher than those obtained by analyzing the high ionization line ratios, which 

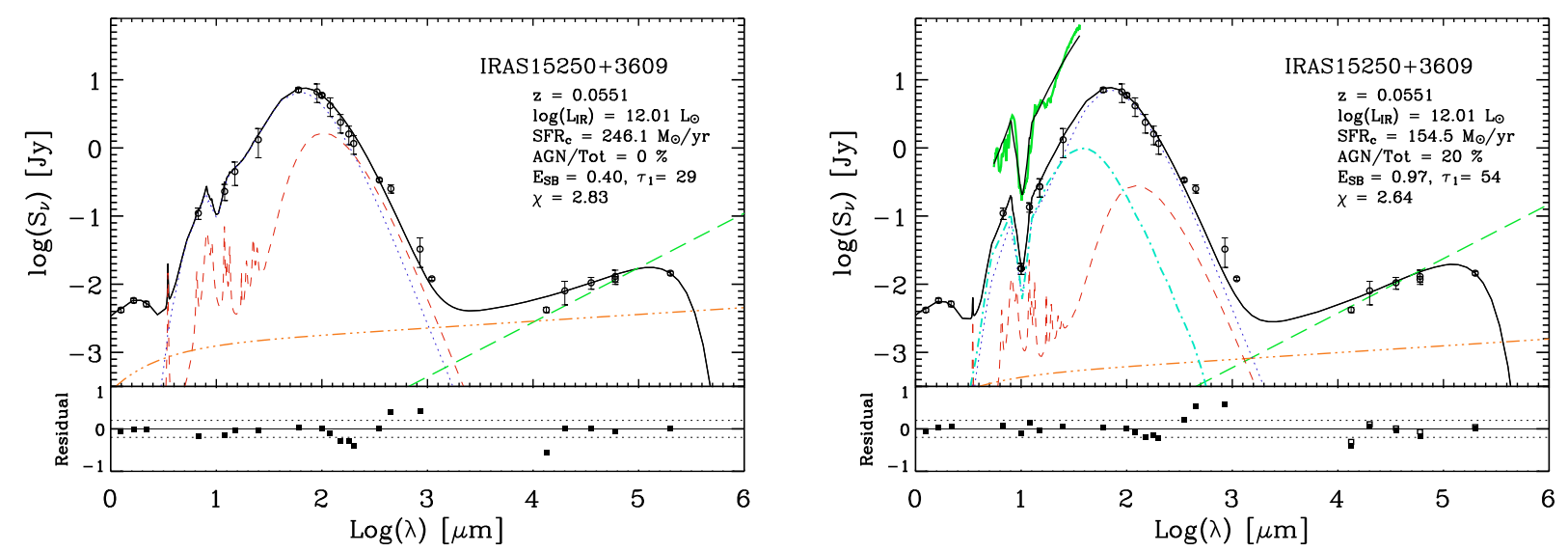

Fig. 6. Left panel: best fit to the IRAS 15250+3609 SED without the inclusion of the Spitzer spectrum. Right panel: best fit to the IRAS $15250+3609$ SED with the inclusion of the Spitzer spectrum. The IRS low resolution spectrum (solid thick line) and the corresponding SED model (solid thin line) are shown upwardly displaced by one dex. The meaning of lines and symbols are the same as in Fig. 1.

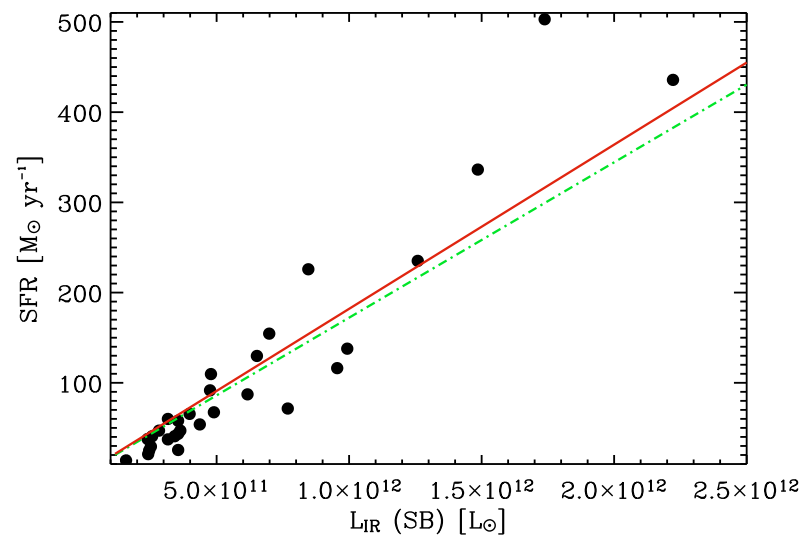

Fig. 7. Correlation between AGN corrected IR luminosity and the derived SFR averaged over the last 10 Myr for our sample of (U)LIRGs. The circles denote the individual galaxies, the solid line represents the best fit to the correlation, and the dashed line represents Kennicutt's relation.

are similar to our values (see appendix for comparisons for individual objects). Therefore, our work supports the notion that neither the mid-infrared slope nor the PAH equivalent widths or relative fluxes provide a good estimate of the AGN contribution (see conclusions in Vega et al. 2005).

Summarizing the above results, we find that the contribution of the AGN is significant and well determined in 10/30 objects $(33 \%)$, while in $5 / 30$ objects it is minimal $(5 \%)$. In the remaining $15 / 30$ objects $(50 \%)$ the SED can be well fitted by almost pure starbursts because the total contribution of the AGN is $<1 \%$, less than the accuracy of the model in all bands. Even in the objects where the presence of the AGN is significant, only in one case, IRAS $08572+3915$, does the AGN dominate the bolometric luminosity. In general, the optical spectral types of the galaxies (Col. 9 in Table 1) agree very well with the results from the SED analysis. All galaxies classified as "HII" were well modelled with pure SB models, while those classified as "Sy" needed some AGN contribution. The situation is less clear for galaxies classified as Liners. Some of them (4) were modelled with pure SB models, while 5 of them, IRAS 143481447, IRAS 15250+3609, IRAS 01364-1042, CGCG 436-30 and NGC 6286, required the presence of a buried AGN.

Since only 11/30 objects have published Spitzer IRS data that can be used to add further constraints to our fitting procedure, we cannot definitely rule out that the AGN contribution is larger, and in particular, that our conclusions on some pure starbursts need revision. It is, however, less likely that the inclusion of Spitzer spectra changes our conclusion of the overall dominant contribution of the SB in (U)LIRGs (see Sect. 5.2).

\subsection{SED fits at radio frequencies}

In Paper I we found a systematic deficit of the $22 \mathrm{GHz}$ emission in our galaxies when compared to that expected from the radio spectral slope calculated at lower frequencies (Clemens et al. 2008). Figure 1 shows that the $22 \mathrm{GHz}$ data fall below the best fit model in 19/30 of the galaxies. In 16 out of 19 objets the differences between models and $22 \mathrm{GHz}$ data are small, most of them within 20-30\%, as can be seen from the residual plots of the same figure. For 9 objects the $22 \mathrm{GHz}$ deficit is within the observational error. For the other 7 we notice that the $22 \mathrm{GHz}$ flux, though below the total predicted radio emission, falls above the predicted synchrotron emission (long dashed line in the plots). Thus a possible explanation is that the deficit at $22 \mathrm{GHz}$ may be due a deficit of thermal emission caused by absorption of ionizing photons by dust within the HII regions (Valdés et al. 2005). In the above 7 objects we estimate that the fractional absorption is between $20 \%$ to $40 \%$, as can be seen from the open squares in the residual plots of Fig. 1. Finally, three galaxies, UGC 8387, IZW 107 and Arp 199, show a deficit in the high frequency radio emission that cannot be explained by observational errors (unless we have severely underestimated them) or by dust absorption. We are currently investigating the origin of this discrepancy (Clemens et al., in preparation).

Two galaxies of the sample, UGC 8058 and UGC 8696, show variability at radio frequencies (Fig. 1, Paper I) on a timescale of a few years (Condon et al. 1991). In the case of UGC 8058 the higher radio fluxes in Fig. 1 are from observations taken on the same day. They are higher by about 0.4 dex and have a slope which is strikingly similar to that at the lower radio fluxes. These data result from observations taken during the last 15 years. The variability may be an indication that the AGN actually powers sporadic emission which dominates at radio frequencies (Lonsdale et al. 2003). Since the higher fluxes have been obtained from observations taken on the same day, and the lower fluxes refer to a much longer period, the low scatter of the latter suggests that they may constitute the genuine emission of the starburst. In the fitting procedure we have thus assumed that 


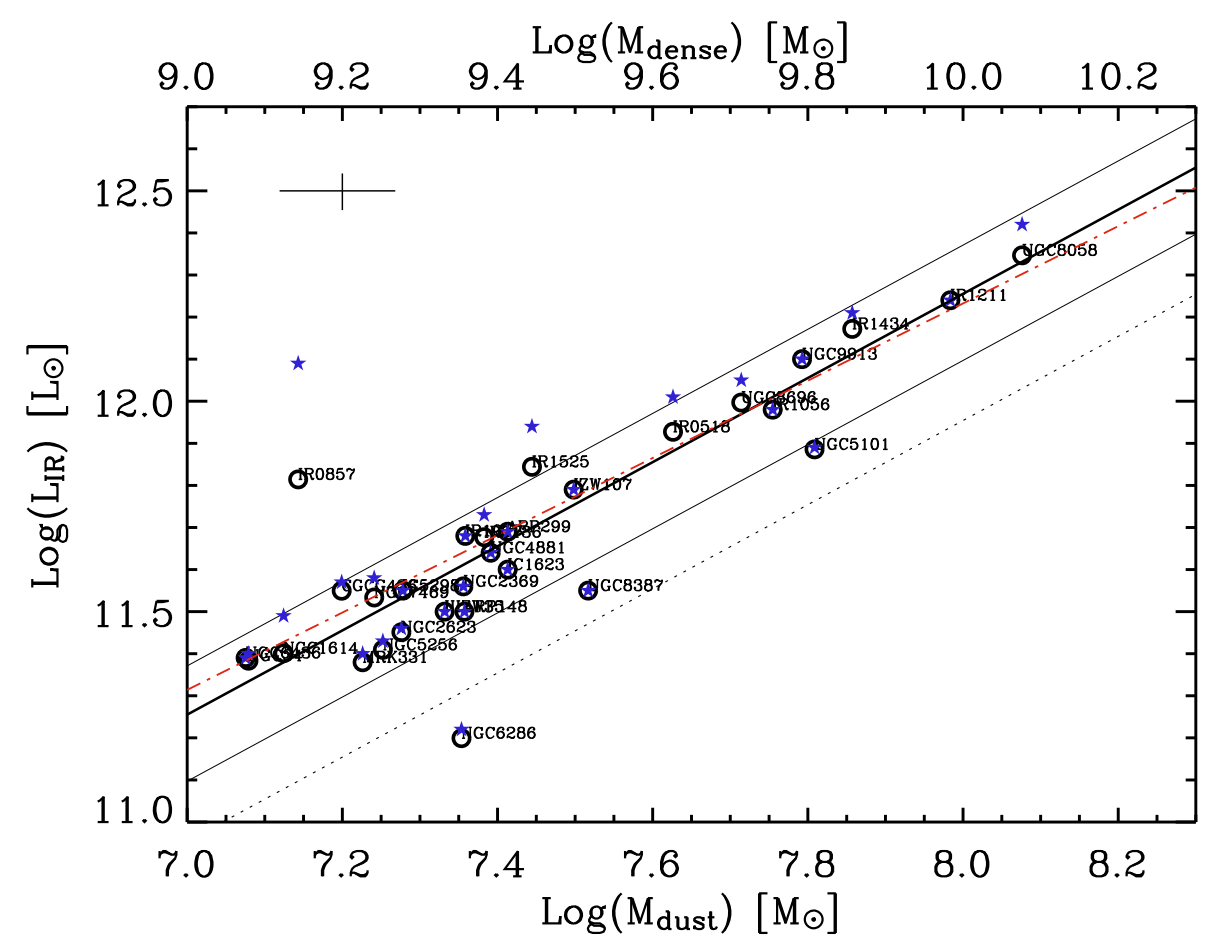

Fig. 8. Correlation between the dust mass in the dense component, derived from our best fit models, and the IR luminosity for our sample of 30 (U)LIRGs. On the upper axis, we display the equivalent molecular mass for a dust-to-gas ratio of 100. Filled stars denote the total IR luminosity, calculated from the 4 IRAS bands (Sanders \& Mirabel 1996), while the black open circles denote the IR luminosity corrected for the AGN contribution. The latter was obtained by integrating the luminosity of the starburst component of the best-fit model. The thick solid line is a linear fit to this AGN corrected IR luminosity, i.e. the IR luminosity due to the starburst emission component, while the dot-dashed line corresponds to the fit to the total IR luminosity. See text for details. The dotted line corresponds to the fit between the IR luminosity and the dense molecular mass as traced by HCN emission obtained by Gao \& Solomon (2004a). The cross in the upper left denotes the typical errors of the IR luminosities and masses derived with our models as explained in Sect. 4.

the lower level radio emission is representative of the starburst. Both galaxies have published IRS spectra, which, together with the broad band SED, are well fitted by a composite model, SB plus AGN.

\subsection{Molecular masses in very actively star forming galaxies}

Our SED fitting technique allows a fairly accurate determination of the main physical parameters of the power sources in compact (U)LIRGs, and in particular, a full characterization of the starburst component. One of the quantities that we derive directly from the fit is the mass of the obscuring dust present in the dense molecular clouds. This can be converted into a mass of dense molecular gas associated with star formation, after assuming a suitable gas/dust mass ratio. This method of determining the mass of dense molecular gas in galaxies with high starformation rates may constitute a viable alternative to the more traditional methods based on the molecular tracers of high density regions (e.g. HCN, see Gao \& Solomon 2004a, and references therein).

In order to obtain molecular gas masses from the dust masses, we have adopted a conservative value of $G / D=100$ which is typical of nearby star forming galaxies. This value seems adequate for our sample because there is evidence that the metallicity in these objects is around solar (Rupke et al. 2007). Furthermore, our chemical evolution models indicate that the metallicity of the gas changes by less than $30 \%$ during the whole duration of the burst. This allows us to assume a constant dust to gas ratio for all the objects, independent of their evolutionary status.
In Fig. 8 we plot the infrared luminosity $(8-1000 \mu \mathrm{m})$ against the values of the dust masses of our objects. On the upper horizontal axis we show the corresponding molecular masses, obtained by assuming $G / D=100$. The existence of the tight linear correlation between the infrared luminosity and the dust mass of the dense star forming component, reproduced in Fig. 8, is a natural consequence of the fact that the infrared emission in our objects is dominated by the MC component. The correlation coefficient is $R^{2}=0.874$, and the non-parametric Kendall tau coefficient is $\tau_{\mathrm{K}}=0.74$, indicating a very low probability of non-correlation. Assuming $G / D=100$ we find that the best-fit (logarithmic) relation for the galaxies of our sample is

$\log \left(L_{\mathrm{IR}}^{\mathrm{SB}}\right)=(1.01 \pm 0.08) \log \left(M_{\mathrm{den}}\right)+(2.20 \pm 0.79)$

or, in linear form,

$L_{\mathrm{IR}}^{\mathrm{SB}} / M_{\mathrm{den}}=183( \pm 11) L_{\odot} M_{\odot}^{-1}$.

This relation is plotted in the figure as a thick solid line, while the thin solid lines correspond to the one-sigma deviation around the mean relation.

It is also remarkable that objects with a significant contribution from an AGN lie above this relation. However, when the contribution of the AGN to the IR luminosity is subtracted, these objects closely fit the relation. The unique exception is IRAS $08572+3915$ which seems to require an IR contribution from the AGN even higher than the value of $47 \%$ obtained from the SED fit.

All these findings support the existence of a fairly robust correlation between the infrared luminosity and dust/gas mass of the star forming component, which could possibly be used to 
Table 10. Molecular data for the galaxies in common between the Gao \& Solomon (2004a) sample and our sample of (U)LIRGs. Column (1) lists the name of the galaxy; (2) the luminosity distance in Mpc; (3) the total $L_{\mathrm{IR}}$, in units of $10^{11} L_{\odot}$, given by Gao \& Solomon (2004a) but corrected for our different luminosity distance; (4) the $L_{\mathrm{IR}}$ due to the starburst component from our fitted model; (5) the $L_{\mathrm{HCN}}$ from Gao \& Solomon, in units of $10^{8} \mathrm{~K} \mathrm{~km} \mathrm{~s}^{-1} \mathrm{pc}^{2}$, and corrected using our calculated luminosity distance; and (6) the $\alpha_{\mathrm{HCN}}$ needed to convert our molecular mass (Col. 4 in Table 6) to the observed $L_{\mathrm{HCN}}$.

\begin{tabular}{lccccc}
\hline \hline Name & $\begin{array}{c}D_{\mathrm{L}} \\
(\mathrm{Mpc})\end{array}$ & $\begin{array}{c}L_{\mathrm{IR}} \\
\left(10^{11} L_{\odot}\right)\end{array}$ & $\begin{array}{c}L_{\mathrm{IR}}^{\mathrm{SB}} \\
\left(10^{1 \mathrm{R}} L_{\odot}\right)\end{array}$ & $\begin{array}{c}L_{\mathrm{HCN}} \\
\left(10^{8} \mathrm{~K} \mathrm{~km} \mathrm{~s}^{-1} \mathrm{pc}^{2}\right)\end{array}$ & $\begin{array}{c}\alpha_{\mathrm{HCN}} \\
\left(M_{\odot} / \mathrm{K} \mathrm{km} \mathrm{s}^{-1} \mathrm{pc}^{2}\right)\end{array}$ \\
\hline UGC 8058 & 173.987 & 31.678 & 22.243 & 19.414 & 6.190 \\
IR 12113+0305 & 309.289 & 19.000 & 19.000 & 14.1 & 6.772 \\
IR 05189-2524 & 175.661 & 12.565 & 5.943 & 6.596 & 6.320 \\
UGC 9913 & 73.465 & 13.560 & 13.560 & 8.898 & 6.929 \\
UGC 8696 & 155.376 & 13.537 & 10.204 & 13.57 & 3.780 \\
IR 10565+2448 & 177.947 & 9.889 & 9.889 & 10.754 & 5.229 \\
UGC 5101 & 162.193 & 9.143 & 7.705 & 10.250 & 6.298 \\
Arp 299 & 41.500 & 5.849 & 5.849 & 1.956 & 13.140 \\
UGC 4881 & 161.812 & 4.520 & 4.520 & 3.758 & 6.531 \\
IC 1623 & 81.452 & 4.641 & 4.641 & 8.448 & 3.100 \\
NGC 1614 & 64.489 & 4.019 & 3.507 & 1.301 & 10.133 \\
UGC 8387 & 95.008 & 3.918 & 3.918 & 9.979 & 3.318 \\
NGC 7469 & 66.000 & 3.802 & 3.548 & 2.094 & 8.298 \\
Arp 148 & 141.647 & 3.566 & 3.566 & 3.908 & 5.802 \\
Mrk 331 & 74.931 & 2.663 & 2.419 & 3.317 & 5.120 \\
\hline
\end{tabular}

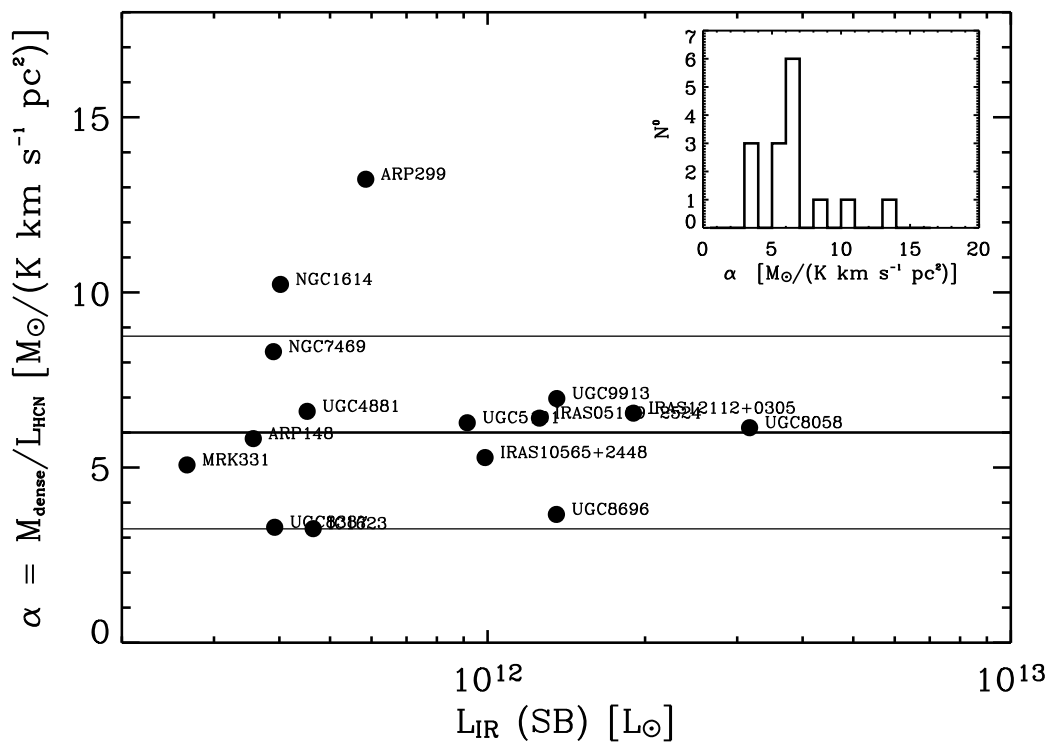

Fig. 9. Values of $\alpha_{\mathrm{HCN}}$ for the 15 galaxies in common between the Gao \& Solomon sample and our sample of (U)LIRGs as a function of the starburst IR luminosity. The filled circles are the data, the thick solid line represents the median value of $\alpha_{\mathrm{HCN}}$, the thin solid lines represent the derived $\sigma$ of the distribution in $\alpha_{\mathrm{HCN}}$. The histogram of the distribution is shown to the upper right.

determine the fractional contribution of the AGN in luminous IR objects where the gas mass is known by other methods.

As a check of our finding, we have compared our results with that obtained by Gao \& Solomon (2004a) for their sample of 64 star forming galaxies. For these objects, they derived a tight correlation between the HCN luminosity and the IR luminosity, that was interpreted as evidence that both originate in the same region. They obtained the mass of dense molecular gas from the observed $L_{\mathrm{HCN}}$ by adopting a conversion factor $M_{\text {dense }} / L_{\mathrm{HCN}}$

$\alpha_{\mathrm{HCN}}=2.1 \sqrt{n\left(H_{2}\right)} / T_{\mathrm{b}}=10 M_{\odot}\left(\mathrm{K} \mathrm{km} \mathrm{s}^{-1} \mathrm{pc}^{2}\right)^{-1}$,

assuming a typical density of $n\left(\mathrm{H}_{2}\right) \sim 3 \times 10^{4} \mathrm{~cm}^{-3}$ and brightness temperature $T_{\mathrm{b}}=35 \mathrm{~K}$. Their relation between IR luminosity and dense mass is shown in Fig. 8 as a dotted line:

$L_{\mathrm{IR}}^{\mathrm{SB}} / M_{\mathrm{den}}=90 L_{\odot} M_{\odot}^{-1}$.
Their relation falls below our relation by about a factor of two. In order to understand the origin of this discrepancy we searched the Gao \& Solomon sample for common objects and derived the conversion factors, $\alpha_{\mathrm{HCN}}$, between our estimated gas masses and the HCN luminosities quoted by them.

Table 10 lists the values of the IR and HCN luminosities of the 15 common galaxies ${ }^{5}$ while Fig. 9 shows the derived $\alpha_{\mathrm{HCN}}$ against the IR luminosity. The median value of $\alpha_{\mathrm{HCN}}$ for the common objects is $6.0 M_{\odot}\left(\mathrm{K} \mathrm{km} \mathrm{s}^{-1} \mathrm{pc}^{2}\right)^{-1}$ with $87 \%$ of the galaxies having $\alpha_{\mathrm{HCN}}=6 . \pm 2.75$. The exceptions are Arp 299, and NGC 1614 that are also outliers in the Gao \& Solomon correlation. These galaxies have values of $L_{\mathrm{IR}} / L_{\mathrm{HCN}} \sim 3000$, while

5 There are 14 galaxies in common between the two samples. We also included in the study IRAS $12112+0305$ from the Graciá-Carpio et al. (2006) sample. 
the mean value of $L_{\mathrm{IR}} / L_{\mathrm{HCN}}$ in their sample is 900-1200, implying significantly weaker $\mathrm{HCN}$ emission.

Our median value of $\alpha_{\mathrm{HCN}}$ is about half that derived by Gao \& Solomon (2004a) for their full sample. However the $\alpha_{\mathrm{HCN}}$ factor derived by Gao \& Solomon (2004a) is certainly biased towards normal galaxies, that constitute the majority of their sample, and may not be representative of the more extreme conditions of the molecular gas in (U)LIRGs. That a lower $\alpha_{\mathrm{HCN}}$ factor would be more appropriate for (U)LIRGs was also argued by Gao \& Solomon (2004a), who noticed that this factor is a sensitive function of the brightness temperature, $T_{\mathrm{b}}$, that can be higher in (U)LIRGs. Indeed, that conversion factors between molecular gas mass and luminosity may be lower in more extreme star forming conditions has already been found for CO emission (Solomon et al. 1997; Downes \& Solomon 1998). Moreover brightness temperatures reaching hundreds of kelvins have been found in massive Galactic star forming cores (Boonman et al. 2001).

Making the same approximation as Gao \& Solomon, that the $\mathrm{HCN}$ emission originates in gravitationally bound cloud cores, and assuming the same core density of $n\left(\mathrm{H}_{2}\right) \sim 3 \times 10^{4} \mathrm{~cm}^{-3}$, we estimate from Eq. (8) that our $\alpha_{\mathrm{HCN}}$ would require a $T_{\mathrm{b}} \sim$ $65_{-21}^{+28} \mathrm{~K}$.

An accurate determination of the conversion factor between $\mathrm{HCN}$ and the mass of the very dense molecular gas needs more extensive studies, including observations of HCN lines at higher frequencies. In this respect, we anticipate that preliminary results of the combined analysis of the SED and the high excitation HCN transitions in Arp 220 (Vega et al., in preparation) indicate that these high brightness temperatures are likely, but also that other factors (abundances, densities, velocity gradients) affect the derived value of $\alpha_{\mathrm{HCN}}$. Greve et al. (2006), in their very extensive study of the molecular emission in Arp 220, derived a likely range of dense molecular masses of [0.6-2.0] $\times 10^{10} M_{\odot}$. Their derived lower limit corresponds to our derived value. We notice also that the upper limit of Greve et al. exceeds the dynamical mass estimated by Downes \& Solomon (1998).

IRAS $08572+3915$ falls significantly above the correlation, suggesting that we could have overestimated the SB contribution. However, a further decrease of the SB component is incompatible with the radio SED, dominated by thermal emission, so that a fraction of it should be attributed to the AGN. Alternatively, the starburst in IRAS $08572+3915$ could be characterized by a metallicity significantly lower than the average in the sample. In fact, while the typical metallicity of (U)LIRGs is 1.1 solar (Rupke et al. 2007), that of IRAS $08572+3915$ is only 0.8 solar (as obtained from the N2 calibrator, Denicoló et al. 2002; and optical emission lines Veilleux et al. 1995). In this case, the thermal radio emission is enhanced (with respect to FIR emission) by about $10 \%$, but, and more importantly, its dense gas mass will be $40 \%$ higher than that derived from the assumed dust/gas ratio. This would push the galaxy on the border of the $L_{\mathrm{IR}}-M_{\mathrm{gas}}$ correlation.

\subsection{The star forming efficiency, SFE}

With the ratio between the current star formation rate and the mass of dense gas, we obtain a quantity that measures the reciprocal of the SF time scale and that is usually called star formation efficiency (SFE, Gao et al. 2004a). This ratio is plotted in the different panels of Fig. 10. There is only a weak correlation between the SFE and the IR luminosity of the SB or the mass of dense gas, while an anti-correlation becomes fairly evident (Kendall coefficient, $\tau_{\mathrm{K}}=-0.85$ ) when the SFE is plotted against the evolutionary phase of the SB (bottom left panel). The efficiency of the star formation is greater in the earlypeak phases of the starburst $\left(E_{\mathrm{SB}} \lesssim 1\right.$.) and then tails off to values characteristic of more normal galaxies (Gao \& Solomon 2004) as the starburst reaches the later phases.

$\log (S F E)=-(0.18 \pm 0.02) \cdot E_{\mathrm{SB}}+(1.79 \pm 0.05)$.

Stars in the bottom panels mark objects in common with Gao \& Solomon (2004b). The right panel shows that this anticorrelation exists even adopting the gas masses derived by them, although with a larger scatter. This shows that the existence of this anti-correlation is not a spurious result of the method we have used to determine the gas mass. On the contrary, we are confident that our method provides more reliable SFR and gas mass estimates than those from Gao \& Solomon (2004a), because it reduces the scatter in spite of increasing the number of objects. We notice also that Gao \& Solomon (2004a) claim that the star formation rate per unit mass of dense gas is constant for all galaxies, including ULIRGs, and that the star formation law in terms of dense molecular content is well represented by a power law with exponent $\sim 1.0$.

Such a rapid decrease of the star formation efficiency as the starburst evolves strongly suggests that feedback plays a major role in the evolution of these extreme objects. To check whether there are significant differences between pure and mixed ULIRGs, we have plotted the former objects with filled symbols and the latter objects with open symbols. In the lower left panel of Fig. 10 there appears to be a separation between the two classes of ULIRGs, with the efficiency in early mixed objects being on average $\sim 40 \%$ larger than in early pure starbursts. Given the small number of objects, this is far from being a definite conclusion. However, if confirmed, it will shed important new light on the AGN-Starburst connection. At present, we cannot say whether a higher efficiency in mixed (U)LIRGs is due to an enhancement of the SFR due to positive AGN feedback, or a lower mass of dense gas, due to negative AGN feedback.

While $E_{\mathrm{SB}}\left(=\mathrm{Age}_{\mathrm{b}} / t_{\mathrm{b}}\right)$ is a quantity entirely derived from our best fit models it would be desirable to put the above finding on observational grounds, to check its independence from the modelling process. As already mentioned, Vega et al. (2005) found a tight correlation between $E_{\mathrm{SB}}$ and the FIR colour $f_{60} / f_{100}$. This correlations goes in the sense that as the $\mathrm{SB}$ evolves, i.e. $E_{\mathrm{SB}}$ increases, the infrared colour gets cooler, i.e. $f_{60} / f_{100}$ decreases.

Indeed, by using the FIR colour instead of our indicator of the evolutive phase of the starburst, we obtain the correlation shown in Fig. 11. A Kendall test indicates a very strong correlation between the SFE and the IR colours corrected for the AGN contribution, $\tau_{\mathrm{K}}=0.78$. This correlation remains strong $\left(\tau_{\mathrm{K}}=0.77\right)$ if the observed IR colors, i.e. without AGN contribution correction, are used.

$\log (S F E)=(1.74 \pm 0.20) \cdot \log \left(f_{60} / f_{100}\right)+(1.55 \pm 0.03)$.

Instead of SFE we could have used the ratio LIR/ $L_{\mathrm{HCN}}$ and obtained a correlation analogous to that shown in Fig. 9a of Gao \& Solomon (2004). Considering only ULIRGs in this figure, a definite trend of decreasing efficiency at cooler $f_{60} / f_{100}$ colour, with slope $\sim 1.72$, is seen. Therefore, this strong correlation between the observed colour and the observed SFE indicates that this variation of the SFE is real. The additional information on the evolutive phase of the starburst and on the AGN contamination, allowed by a realistic modelling procedure, eliminates a large part of the dispersion in the star formation efficiency found by Gao \& Solomon (2004). 

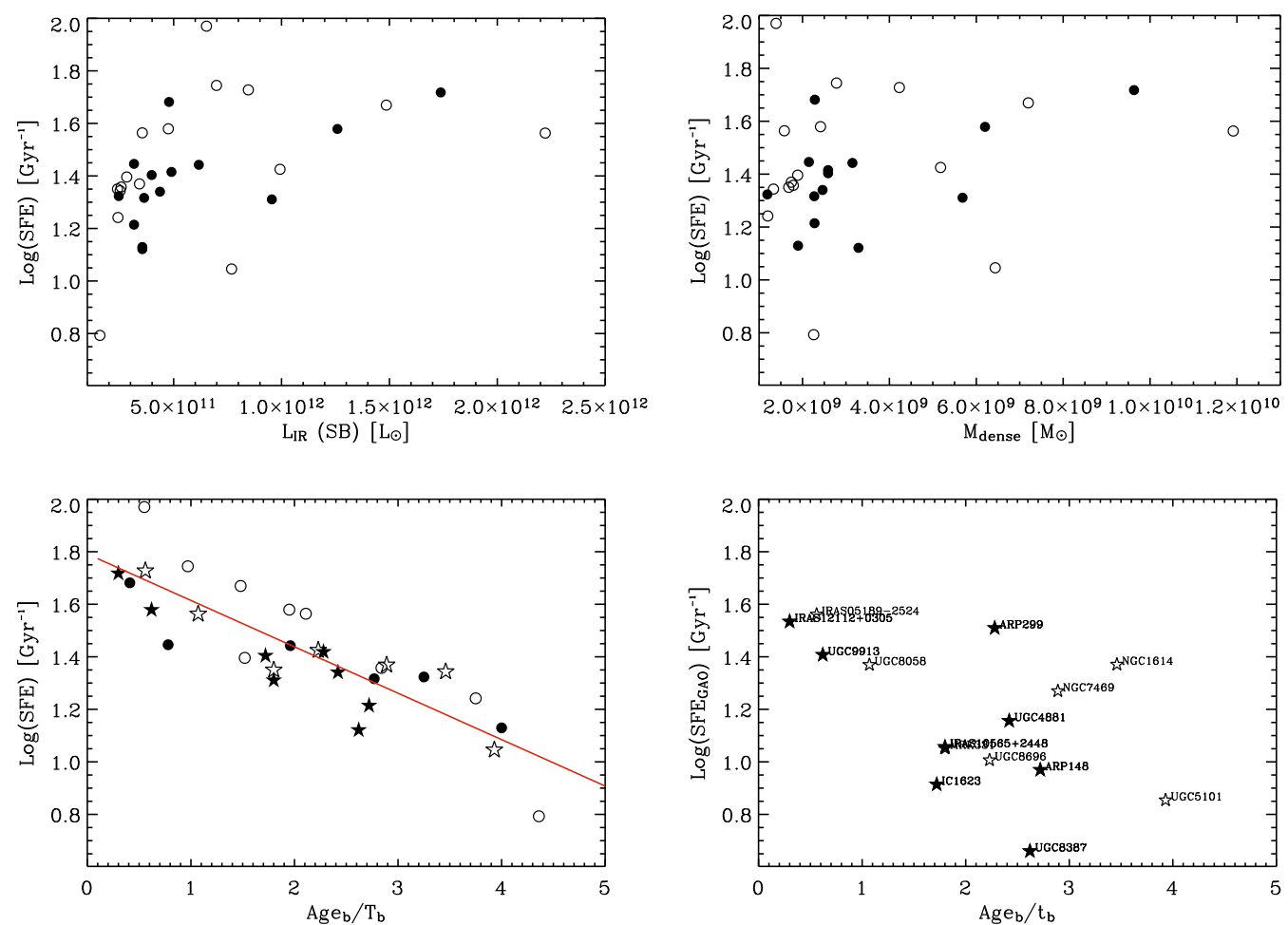

Fig. 10. Correlations of the SFE with different quantities: upper left: correlation of AGN corrected IR luminosity from the best-fit models and the derived SFE for our sample of (U)LIRGs. Upper right: SFE vs. the derived dense gas masses of the models. Lower left: SFE as derived from our models versus the evolutive phase of the starburst. Galaxies in common with the Gao \& Solomon sample are marked as stars. Filled symbols correspond to galaxies fitted with pure SB models while open symbols correspond to those fitted with mixed (AGN + SB) models. Lower right: as adjacent panel but for the subset of galaxies in common with the Gao \& Solomon sample and calculated using their derived molecular gas mass and our estimate of the recent SFR. Filled stars correspond to galaxies fitted with pure SB models while open stars correspond to those fitted with mixed models.

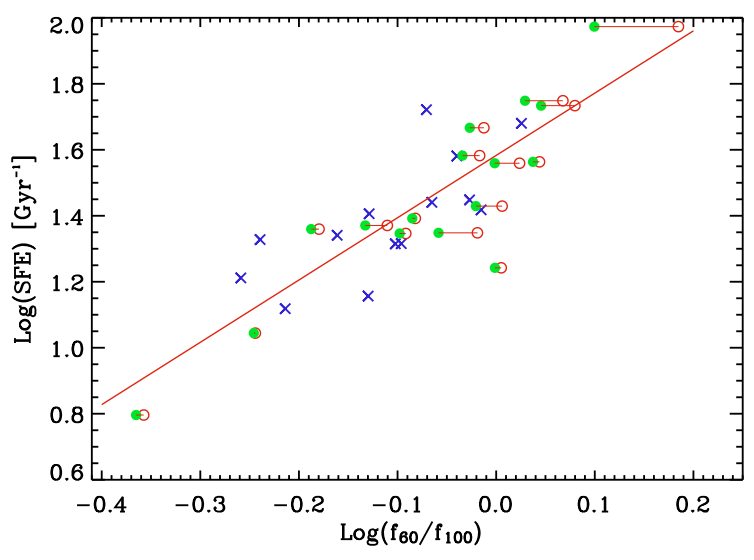

Fig. 11. Correlation between the far-infrared color $\left(f_{60} / f_{100}\right)$ and the derived SFE for our sample of (U)LIRGs. The meaning of the symbols are the same as in Fig. 5. The thick solid line is the best fit to this correlation.

\subsection{Implications for the evolution of LIRGs and ULIRGs}

All but one of the 30 compact (U)LIRGs we have analyzed are predominantly powered by a starburst, contributing more than $80 \%$ of the bolometric light. Sixteen galaxies show evidence for the presence of an AGN, but only nine have an AGN contribution that exceeds $10 \%$ of the IR luminosity, although it may be larger at NIR-MIR wavelengths.

Since there is much evidence that IRAS bright galaxies are associated with gas-rich mergers, this could suggest that strong AGN activity is only triggered in certain types of mergers. Our sample is biased toward IRAS bright objects, and the mergers involved should be those in which disruption and/or infall of gas is expected, such as discussed by Sopp et al. (1990). However, modelling of such encounters does not indicate that the dynamical parameters (prograde, retrograde orbit etc) influence the fueling of an active nucleus, at least for Seyfert galaxies (Keel 1996).

The alternative explanation is that strong AGN activity is common to most such mergers, but lasts for an order of magnitude less time than the starburst. This would correspond to AGN lifetimes of a few million years. The latter figure is in very good agreement with AGN lifetimes derived from the comparison of the ratio between SNIIb/c and SNII rates in normal spirals and in Seyfert II galaxies (Bressan et al. 2002).

\section{Conclusions}

Based on homogenized literature data and on our own radio observations (Prouton et al. 2004; Clemens et al. 2008) we have constructed well-sampled, broad band, NIR to radio spectral energy distributions of 30 luminous and ultra-luminous infrared galaxies, selected from the sample of compact (U)LIRGs of Condon et al. (1991). For one third of the sample, the broad band SED has also been complemented by the published low resolution Spitzer IRS spectrum. The library of SEDs, available in electronic form (see Tables B.2 and B.3), constitutes a real work bench for models aimed at interpreting such powerful and obscured sources. It is tempting to say that the template SEDs may be used for the analysis of huge star forming objects detected at high redshifts, if it were not that one of the strongest warnings emerging from this work is that it is unreliable to simply 
re-scale observed SEDs to higher luminosities. On the contrary, the aim of the present investigation was to determine physical parameters and environmental conditions that accompany very high star formation rates, that can be used to model high redshift galaxies.

To this end, we have analyzed the observed SEDs with a chemo-spectro-photometric code that includes one of the most advanced and realistic treatments of the complex interaction between star formation, starlight and dust reprocessing (GRASIL). In addition, emission from an AGN, based on the same homogeneous physics, was added in those cases where the pure starburst model failed to fit the data.

Our results can be summarized as follows:

- 16/30 sources show evidence for the presence of an AGN. However only in $9 / 30$ sources does the contribution of the AGN to the total infrared luminosity exceed $10 \%$. Only one galaxy, IRAS $08572+3915$, seems to be powered by an AGN. We therefore find that the fraction of starburst dominated objects (i.e. pure SB galaxies and mixed galaxies with AGN contributions lower than $10 \%$ ) in our sample, is $70 \%$. This value is in good agreement with that found by Farrah et al. (2003).

- In a previous paper we noticed that the results of fitting (U)LIRGs SEDs could be affected by a degeneracy between optical depth of the starburst MCs and the presence of the AGN, since both may affect the MIR emission. By sampling the silicate absorption feature at $9.7 \mu \mathrm{m}$, low resolution Spitzer IRS spectra can actually break this degeneracy and provide a more accurate quantification of the AGN/SB parameters.

The largest revision after including the Spitzer IRS spectrum has been found for IRAS $15250+3609$, whose broad band SED could be fitted by a pure starburst, whereas reproducing also the $9.7 \mu \mathrm{m}$ feature, revealed a dust obscured AGN contributing $20 \%$ of the IR luminosity. The classification of the other three pure starbursts remained unchanged. In those cases where the presence of the AGN was already suspected from other diagnostics, the inclusion of Spitzer IRS observations confirmed this presence and increased the robustness of the derived parameters.

- Our measured AGN fractions are consistent with those reported by Armus et al. (2007) based on the mid-infrared line ratios $\mathrm{Ne}[\mathrm{V}] / \mathrm{Ne}[\mathrm{II}]$ and $\mathrm{O}[\mathrm{IV}] / \mathrm{Ne}[\mathrm{II}]$. These lines may thus provide a fairly accurate measure of the AGN contribution to the bolometric luminosity of infrared luminous galaxies. The PAH equivalent widths and mid-infrared spectral slope on the other hand overestimate (often by large factors) the AGN fraction.

For two galaxies, our findings are markedly different to those of Armus et al., namely IRAS 14348-1447 and IRAS $15250+3609$. For the former, we find an AGN contribution to the IR luminosity of $\sim 10 \%$, while they find no evidence of an AGN from the MIR analysis. Indeed, this AGN contribution is required by its red NIR colour, $J-K=1.71$ which cannot be explained by stellar populations alone. The power source of IRAS $15250+3609$ could not be determined by Armus et al., while our analysis indicates that it is a mixed object with a very obscured AGN, that contributes $\sim 20 \%$ of the IR luminosity.

- If an active nucleus forms part of an evolutionary phase of ULIRGs then we expect this phase to be energetically important for an order of magnitude less time than the starburst. From our modelling, this would correspond to a few Myr. However, there is no evidence in our sample that the AGN phenomenon is correlated with a particular phase of the starburst, although all 4 AGNs in the early phase of the SB make a large IR contribution, while only 1 of the 12 associated with the later SB phases has similar IR power.

- There is evidence that the star formation in objects with an AGN decreases more rapidly than in pure starbursts. In fact, while about $75 \%$ of SBs with AGN have $t_{\mathrm{b}} \leq 20 \mathrm{Myr}$, all pure SBs have $t_{\mathrm{b}} \geq 20$ (and only 3 with $t_{\mathrm{b}}=20$ ). If AGN feedback is the cause it could either be via a positive or negative influence on the star formation process.

- Since the MIR-FIR spectral region in our sample (U)LIRGs is dominated by MC emission, we can determine quite accurately the mass in dust associated with the dense star forming regions. We can convert this to the molecular gas mass, using a typical dust/gas ratio. We find a relation between the infrared luminosity and molecular gas mass with the same slope as that derived by Gao \& Solomon (2004a) from observations of $\mathrm{HCN}$ emission. However, we derive a constant of conversion between HCN flux and mass of dense molecular gas a factor of 2 smaller than that assumed by these authors. Such a low conversion factor for the (U)LIRG population is suggested by Gao \& Solomon (2004a) themselves and by other investigations (e.g. Graciá-Carpio et al. 2006).

Notable outliers from this correlation are those few objects with an energetically important AGN. After correcting their IR luminosity for the AGN contribution, they also lie on the correlation. This is an independent check of the robustness of our method. If confirmed by the study of a larger sample of (U)LIRGs this correlation can be used to evaluate the AGN fraction in distant ULIRGs with known IR and HCN luminosities (e.g. such those observed by Gao et al. 2007).

- Using the ratio between the current SFR and the mass of dense molecular gas we obtain an estimate of the reciprocal of the consumption time that we call efficiency of star formation. While there seems not to exist clear correlations between SFE and IR luminosity of the starburst or mass of dense molecular gas, an evident anti-correlation appears when SFE is plotted against the starburst phase. The SFE decreases by about one order of magnitude as the SB evolves from its early phase to the more advanced phases.

This anti-correlation is not due to a circularity in our fitting method, because it is also evident if we adopt the gas masses obtained by Gao \& Solomon (2004a), although with a larger dispersion. Indeed, most of the dispersion in the star formation efficiency derived observationally from HCN emission (Gao \& Solomon 2004b) can be attributed to this evident fall in efficiency during the lifetime of a starburst.

Acknowledgements. O.V. and M.C. acknowledge the the support of the INAF research fellowships. O.V. also acknowledges the support of the INAOE and the Mexican CONACYT projects 36547 E and 39714 F. A.B., G.L.G. and L.S. acknowledge the warm hospitality of INAOE and the partial funding by the European Community by means of the Maria Curie contract MRTN-CT- 2004503929, MAGPOP. A financial contribution from contract ASI-INAF I/016/07/0 is also acknowledged. We also thank the anonymous referee for a careful and helpful report. This publication makes use of data products from the Two Micron All Sky Survey, which is a joint project of the University of Massachusetts and the Infrared Processing and Analysis Center/California Institute of Technology, funded by the National Aeronautics and Space Administration and the National Science Foundation. This work is also based on data taken with the Spitzer Space Telescope, which is operated by the JPL, Caltech, under a contract with NASA. 


\section{References}

Albrecht, M., Krügel, E., \& Chini, R. 2007, A\&A, 462, 575

Alonso-Herrero, A., Rieke, G. H., Rieke, M. J., \& Scoville, N. Z. 2000, ApJ, 532,845

Alonso-Herrero, A., Quillen, A. C., Simpson, C., Efstathiou, A., \& Ward, M. J. 2001, AJ, 121, 1369

Alonso-Herrero, A., Rieke, G. H., Rieke, M. J., \& Scoville, N. Z. 2002, AJ, 124, 166

Alonso-Herrero, A., Quillen, A. C., Rieke, G. H., Ivanov, V. D., \& Efstathiou, A. 2003, AJ, 126, 81

Anantharamaiah, K. R., Viallefond, F., Mohan, N. R., Goss, W. M., \& Zhao, J. H. 2000, ApJ, 537, 613

Armus, L., Heckman, T. M., \& Miley, G. K. 1989, ApJ, 347, 727

Armus, L., Charmandaris, V., Spoon, H. W. W., et al. 2004, ApJS, 154, 178

Armus, L., Charmandaris, V., Bernard-Salas, J., et al. 2007, ApJ, 656, 148

Baan, W. A., Salzer, J. J., \& Lewinter, R. D. 1998, ApJ, 509, 633

Ballo, L., Braito, V., Della Ceca, R., et al. 2004, ApJ, 600, 634

Benford, D. J. 1999, Ph.D. Thesis, California Institute of Technology, Source DAI-B 60/08

Boonman, A. M. S., Stark, R., van der Tak, F. F. S., et al. 2001, ApJ, 553, 63

Braito, V., Della Ceca, R., Piconcelli, E., et al. 2004, A\&A, 420, 79

Brandl, B. R., Bernard-Salas, J., Spoon, H. W. W., et al. 2006, ApJ, 653, 1129

Bressan, A., Granato, G. L., \& Silva, L. 1998, A\&A, 332, 135

Bressan, A., Silva, L., \& Granato, G. L. 2002a, A\&A, 392, 377

Bressan, A., Della Valle, M., \& Marziani, P. 2002b, MNRAS, 331, 25

Carico, D. P., Sanders, D. B., Soifer, B. T., et al. 1988, AJ, 95, 356

Carico, D. P., Keene, J., Soifer, B. T., \& Neugebauer, G. 1992, PASP, 104, 1086

Chapman, J. M., Staveley-Smith, L., Axon, D. J., et al. 1990, MNRAS, 244, 281

Charmandaris, V., Stacey, G. J., \& Gull, G. 2002a, ApJ, 571, 282

Charmandaris, V., Laurent, O., Le Floc'h, E., et al. 2002b, A\&A, 391, 429

Chini, R., Kruegel, E., \& Lemke, R. 1996, A\&AS, 118, 47

Clemens, M. S., \& Alexander, P. 2004, MNRAS, 350, 66

Clemens, M. S., Vega, O., Bressan, A., et al. 2008, A\&A, 477, 95

Colina, L., Arribas, S., \& Monreal-Ibero, A. 2005, ApJ, 621, 725

Condon, J. J., Huang, Z.-P., Yin, Q. F., \& Thuan, T. X. 1991a, ApJ, 378, 65

Condon, J. J., Frayer, D. T., \& Broderick, J. J. 1991b, AJ, 101, 362

Dale, D. A., Silbermann, N. A., Helou, G., et al. 2000, AJ, 120, 583

Dasyra, K. M., Tacconi, L. J., Davies, R. I., et al. 2006, ApJ, 638, 745

Davies, R. I., Tacconi, L. J., \& Genzel, R. 2004, ApJ, 613, 781

de Grijp, M. H. K., Miley, G. K., Lub, J., \& de Jong, T. 1985, Nature, 314, 240

Della Ceca, R., Ballo, L., Tavecchio, F., et al. 2002, ApJ, 581, 9

Denicoló, G., Terlevich, R. J., \& Terlevich, E. 2002, MNRAS, 330, 69

Downes, D., \& Eckart, A. 2007, A\&A, 468, 57

Downes, D., \& Solomon, P. M. 1998, ApJ, 507, 615

Draine, B. T., \& Lee, H. M. 1984, ApJ, 285, 89

Dudley, C. C. 1999, MNRAS, 307, 553

Dunne, L., \& Eales, S. A. 2001, MNRAS, 327, 697

Dunne, L., Eales, S., Edmunds, M., et al. 2000, MNRAS, 315, 115

Dwek, E. 1998, ApJ, 501, 643

Eales, S. A., Becklin, E. E., Hodapp, K.-W., Simons, D. A., \& Wynn-Williams, C. G. 1990, ApJ, 365, 478

Efstathiou, A., \& Rowan-Robinson, M. 1995, MNRAS, 273, 649

Elvis, M., Wilkes, B. J., McDowell, J. C., et al. 1994, ApJS, 95, 1

Farrah, D., Afonso, J., Efstathiou, A., et al. 2003, MNRAS, 343, 585

Farrah, D., Bernard-Salas, J., Spoon, H. W. W., et al. 2007, ApJ, 667, 149

Flores, H., Hammer, F., Elbaz, D., et al. 2004, A\&A, 415, 885

Franceschini, A., Braito, V., Persic, M., et al. 2003, MNRAS, 343, 1181

Frayer, D. T., Ivison, R. J., Smail, I., Yun, M. S., \& Armus, L. 1999, AJ, 118, 139

Gallais, P., Charmandaris, V., Le Floc'h, E., et al. 2004, A\&A, 414, 845

Gao, Y., \& Solomon, P. M. 2004a, ApJ, 606, 271

Gao, Y., \& Solomon, P. M. 2004b, ApJS, 152, 63

Gao, Y., Carilli, C. L., Solomon, P. M., \& Vanden Bout, P. A. 2007, ApJ, 660, 93

Gallagher, S. C., Brandt, W. N., Chartas, G., Garmire, G. P., \& Sambruna, R. M. 2002, ApJ, 569, 655

Genzel, R., Lutz, D., Sturm, E., et al. 1998, ApJ, 498, 579

Genzel, R., Tacconi, L. J., Rigopoulou, D., Lutz, D., \& Tecza, M. 2001, ApJ, 563,527

Goldader, J. D., Joseph, R. D., Doyon, R., \& Sanders, D. B. 1995, ApJ, 444, 97 Goldader, J. D., Joseph, R. D., Doyon, R., \& Sanders, D. B. 1997, ApJ, 474, 104 Graciá-Carpio, J., García-Burillo, S., Planesas, P., \& Colina, L. 2006, ApJ, 640, 135

Granato, G. L., \& Danese, L. 1994, MNRAS, 268, 235

Granato, G. L., Danese, L., \& Franceschini, A. 1997, ApJ, 486, 147

Granato, G. L., Lacey, C. G., Silva, L., et al. 2000, ApJ, 542, 710

Greve, T. R., Papadopoulos, P. P., Gao, Y., \& Radford, S. J. E. 2006 [arXiv: astro-ph/0610378]
Hattori, T., Yoshida, M., Ohtani, H., et al. 2004, AJ, 127, 736

Hwang, Chorng-Yuan, Lo, K. Y., Gao, Y., Gruendl, R. A., \& Lu, N. Y. 1999, ApJ, 511, 17

Imanishi, M., \& Dudley, C. C. 2000, ApJ, 545, 701

Imanishi, M., \& Maloney, P. R. 2003, ApJ, 588, 165

Imanishi, M., Dudley, C. C., \& Maloney, P. R. 2001, ApJ, 558, L93

Imanishi, M., Dudley, C. C., \& Maloney, P. R. 2006, ApJ, 637, 114

Imanishi, M., Dudley, C. C., Maiolino, R., et al. 2007, ApJS, 171, 72

Joyce, R. R., \& Simon, M. 1976, PASP, 88, 870

Joyce, R. R., Knacke, R. F., Simon, M., \& Young, E. 1975, PASP, 87, 683

Keel, W. C. 1996, AJ, 111, 696

Kennicutt, R. C. 1998, ARA\&A, 36, 189

Klaas, U., Haas, M., Müller, S. A. H., et al. 2001, A\&A, 379, 823

Kotilainen, J. K., Reunanen, J., Laine, S., \& Ryder, S. D. 2001, A\&A, 366, 439

Krolik, J. H. 1999, Active galactic nuclei: from the central black hole to the galactic environment (Princeton University Press)

Lahuis, F., Spoon, H. W. W., Tielens, A. G. G. M., et al. 2007, ApJ, 659, 296

Lancon, A., Rocca-Volmerange, B., \& Thuan, T. X. 1996, A\&AS, 115, 253

Laurent, O., Mirabel, I. F., Charmandaris, V., et al. 2000, A\&A, 359, 887

Lebofsky, M. J., \& Rieke, G. H. 1979, ApJ, 229, 111

Le Floc'h, E., Charmandaris, V., Laurent, O., et al. 2002, A\&A, 391, 417

Lonsdale, C. J., Smith, H. J., \& Lonsdale, C. J. 1993, ApJ, 405, 9

Lonsdale, C. J., Lonsdale, C. J., Smith, H. E., \& Diamond, P. J. 2003, ApJ, 592, 804

Lonsdale, C. J., Diamond, P. J., Thrall, H., Smith, H. E., \& Lonsdale, C. J. 2006, ApJ, 647, 185

Lutz, D., Genzel, R., Kunze, D., et al. 1999, Ap\&SS, 266, 85

Maiolino, R., \& Rieke, G. H. 1995, ApJ, 454, 95

Maiolino, R., Marconi, A., \& Oliva, E. 2001a, A\&A, 365, 37

Maiolino, R., Marconi, A., Salvati, M., et al. 2001b, A\&A, 365, 28

Maiolino, R., Comastri, A., Gilli, R., et al. 2003, MNRAS, 344, L59

Maloney, P. R., \& Reynolds, C. S. 2000, ApJ, 545, L23

Mathis, J. S., Rumpl, W., \& Nordsieck, K. H. 1977, ApJ, 217, 425

Miles, J. W., Houck, J. R., \& Hayward, T. L. 1994, ApJ, 425, 37

Miles, J. W., Houck, J. R., Hayward, T. L., \& Ashby, M. L. N. 1996, ApJ, 465, 191

Mirabel, I. F., \& Sanders, D. B. 1988, ApJ, 335, 104

Mizutani, K., Suto, H., \& Maihara, T. 1994, ApJ, 421, 475

Montgomery, A. S., \& Cohen, R. J. 1992, MNRAS, 254, P23

Mouri, H., Taniguchi, Y., Sato, Y., \& Kawara, K. 1998, A\&A, 334, 482

Mulchaey, J., Wilson, A. S., \& Tsvetanov, Z. 1996, ApJS, 102, 309

Murphy, T. W. Jr., Soifer, B. T., Matthews, K., Armus, L., \& Kiger, J. R. 2001, AJ, 121, 97

Nagar, N. M., Wilson, A. S., Falcke, H., Veilleux, S., \& Maiolino, R. 2003, A\&A, 409, 115

Neff, S. G., Hutchings, J. B., Standord, S. A., \& Unger, S. W. 1990, AJ, 99, 1088

Neff, S. G., Ulvestad, J. S., \& Teng, S. H. 2004, ApJ, 611, 186

Nenkova, M., Ivezić, Ž., \& Elitzur, M. 2002, ApJ, 570, L9

Nikolic, B., Alexander, P., Cotter, G., Longair, M., \& Clemens, M. 2003, Ap\&SS, 284,573

Panuzzo, P., Bressan, A., Granato, G. L., Silva, L., \& Danese, L. 2003, A\&A, 409, 99

Papadopoulos, P. P., \& Allen, M. L. 2000, ApJ, 537, 631

Pérez García, A. M., \& Rodríguez Espinosa, J. M. 2001, ApJ, 557, 39

Pier, E. A., \& Krolik, J. H. 1993, ApJ, 418, 673

Pihlström, Y. M., Conway, J. E., Booth, R. S., Diamond, P. J., \& Polatidis, A. G. 2001, A\&A, 377, 413

Poggianti, B. M., \& Wu, H. 2000, ApJ, 529, 157

Poggianti, B. M., Bressan, A., \& Franceschini, A. 2001, ApJ, 550, 195

Portinari, L., Chiosi, C., \& Bressan, A. 1998, A\&A, 334, 505

Prouton, O. R., Bressan, A., Clemens, M., et al. 2004, A\&A, 421, 115

Ptak, A., Heckman, T., Levenson, N. A., Weaver, K., \& Strickland, D. 2003, ApJ, 592,782

Rieke, G. H. 1976, ApJ, 210, 5

Rieke, G. H. 1978, ApJ, 226, 550

Rieke, G. H., \& Low, F. J. 1972, ApJ, 176, 95

Rigopoulou, D., Lawrence, A., \& Rowan-Robinson, M. 1996, MNRAS, 278, 1049

Risaliti, G., Gilli, R., Maiolino, R., \& Salvati, M. 2000, A\&A, 357, 13

Risaliti, G., Maiolino, R., Marconi, A., et al. 2006, MNRAS, 365, 303

Roche, P. F., \& Chandler, C. J. 1993, MNRAS, 265, 486

Roche, P. F., Aitken, D. K., Smith, C. H., \& Ward, M. J. 1991, MNRAS, 248, 606

Rupke, D. S. N., Veilleux, S., \& Baker, A. J. 2007 [arXiv:0708. 1766v1]

Sanders, D. B., \& Mirabel, I. F. 1996, ARA\&A, 34, 749

Sanders, D. B., Soifer, B. T., Elias, J. H., Neugebauer G., \& Matthews, K. 1988, ApJ, 328L, 35

Satyapal, S., Watson, D. M., Pipher, J. L., et al. 1999, ApJ, 516, 704 
Severgnini, P., Risaliti, G., Marconi, A., Maiolino, R., \& Salvati, M. 2001, A\&A, 368,44

Shier, L. M., Rieke, M. J., \& Rieke, G. H. 1996, ApJ, 470, 222

Silva, L. 1999, Ph.D. Thesis, SISSA

Silva, L., Granato, G. L., Bressan, A., \& Danese, L. 1998, ApJ, 509, 103

Smith, D. A., Herter, T., Haynes, M. P., Beichman, C. A., \& Gautier, T. N., III 1995, ApJ, 439, 623

Smith, H. E., Lonsdale, C. J., Lonsdale, C. J., \& Diamond, P. J. 1998a, ApJ, 493, L17

Smith, H. E., Lonsdale, C. J., \& Lonsdale, C. J. 1998b, ApJ, 492, 137

Solomon, P. M., Downes, D., Radford, S. J. E., \& Barrett, J. W. 1997, ApJ, 478, 144

Soifer, B. T., Neugebauer, G., Matthews, K., et al. 2000, AJ, 119, 509

Soifer, B. T., Neugebauer, G., Matthews, K., et al. 2001, AJ, 122, 1213

Soifer, B. T., Neugebauer, G., Matthews, K., Egami, E., \& Weinberger, A. J. 2002, AJ, 124, 2980

Soifer, B. T., Bock, J. J., Marsh, K., et al. 2003, AJ, 126, 143

Sopp, H., Alexander, P., \& Riley, J. 1990, MNRAS, 246, 143

Spinoglio, L., Benedettini, M., de Troia, G., et al. 2000, ESASP, 456, 261

Spoon, H. W. W., Keane, J. V., Tielens, A. G. G. M., et al. 2002, A\&A, 385, 1022
Stickel, M., Lemke, D., Klaas, U., Krause, O., \& Egner, S. 2004, A\&A, 422, 39

Surace, J. A., \& Sanders, D. B. 2000, AJ, 120, 604

Surace, J. A., Sanders, D. B., \& Evans, A. S. 2000, ApJ, 529, 70

Schweitzer, M., Lutz, D., Sturm, E., et al. 2006, ApJ, 649, 79

Takagi, T., Arimoto, N., \& Hanami, H. 2003, MNRAS, 340, 813

Takeuchi, T. T., Buat, V., Iglesias-Páramo, J., Boselli, A., \& Burgarella, D. 2005, A\&A, 432, 423

Taylor, G. B., Silver, C. S., Ulvestad, J. S., \& Carilli, C. L. 1999, ApJ, 519, 185 Vacca, W. D., \& Conti, P. S. 1992, ApJ, 401, 543

Valdés, J. R., Berta, S., Bressan, A., et al. 2005, A\&A, 434, 149

Vega, O., Silva, L., Panuzzo, P., et al. 2005, MNRAS, 364, 1286

Veilleux, S., Kim, D. C., Sanders, D. B., Mazzarella, J. M., \& Soifer, B. T. 1995, ApJS, 98, 171

Veilleux, S., Sanders, D. B., \& Kim, D. C. 1999, ApJ, 522, 139

Véron-Cetty, M. P., \& Véron, P. 1991, ESO, Sci. Rep., 10

Wang, J., Heckman, T. M., Weaver, K. A., \& Armus, L. 1997, ApJ, 474, 659

Weedman, D. W., Hao, L., Higdon, S. J. U., et al. 2005, ApJ, 633, 706

Wu, H., Zou, Z. L., Xia, X. Y., \& Deng, Z. G. 1998, A\&AS, 132, 181

Zezas, A., Ward, M. J., \& Murray, S. S. 2003, ApJ, 594, L31 


\section{Appendix A: Individual objects}

Below we compare our results with the relevant literature concerning the power sources for each of our sample objects. The AGN contributions described refer to the wavelength range 8-1000 $\mu \mathrm{m}$.

\section{UGC 08058 (Mrk 231)}

The identification of this source as the nearest broad absorption line quasar is supported by a wide range of observational data. Compact radio emission from its nucleus (Lonsdale et al. 2003), its X-ray luminosity (Maloney \& Reynolds 2000), short term variability at X-ray wavelengths (Gallagher et al. 2002) and low $3.3 \mu \mathrm{m}$ PAH feature equivalent width (Imanishi \& Dudley 2000) all point to the presence of an AGN.

However, a central starburst is also probably present and accounts for a significant fraction of the soft X-ray luminosity (Braito et al. 2004). Davies et al. (2004) estimate that starformation in the nuclear disk accounts for $25-40 \%$ of the bolometric luminosity. Taylor et al. (1999) use high-resolution multi-wavelength radio observations to deduce that the central starburst component forms stars at a rate of $220 M_{\odot} \mathrm{yr}^{-1}$.

Condon et al. (1991) classify the source as AGN-dominated based on the radio morphology and infrared-radio flux ratio, as do Lutz et al. (1999) using mid-infrared spectroscopy. Farrah et al. (2003) require a 30\% contribution from an AGN for their SED fit. An AGN fraction of $<10 \%$ is found by Armus et al. (2007) using the line ratios $\mathrm{Ne}[\mathrm{V}] / \mathrm{Ne}[\mathrm{II}]$ and $\mathrm{O}[\mathrm{IV}] / \mathrm{Ne}[\mathrm{II}]$, whereas values of close to $100 \%$ are obtained from the PAH equivalent widths or the mid-infrared spectral slope.

Our best fit to the SED plus the IRS spectrum requires an active nucleus accounting for $16 \%$ of the infrared luminosity. Notice however that our fit is based on the assumption that the lower radio fluxes are due to the SB.

\section{IRAS 14348-1447}

The source lies at a position in the mid-infrared diagnostic diagram of Genzel et al. (1998) that suggests an AGN contribution $\$ 25 \%$, and both Lutz et al. (1999) and Charmandaris et al. (2002a,b) classify this source as starburst dominated, based on mid-infrared ISO data.

Risaliti et al. (2006) find a high equivalent width for the $3.3 \mu \mathrm{m}$ PAH feature and flat near-infrared continuum in both nuclei, suggesting a starburst origin, although Imanishi et al. (2006) find weak evidence for the presence of and AGN in the NE nucleus using similar data. Franceschini et al. (2003) find no evidence for the presence of an AGN at hard X-ray wavelengths.

Takagi et al. (2003) model the uv to far-infrared SED with a starburst model with a current star formation rate of $327 M_{\odot} \mathrm{yr}^{-1}$ but do not consider an AGN component.

Farrah et al. (2003) find that this source has an AGN accounting for $17 \%$ of the IR luminosity. Armus et al. (2007) estimate the AGN fraction using Spitzer data to be between $<1 \%$ ( $\mathrm{Ne}[\mathrm{V}] / \mathrm{Ne}[\mathrm{II}]$ line ratio) and $65 \%$ (mid-IR slope). Imanishi et al. (2007) do not find AGN signatures in the Spitzer spectrum but find strong evidence of the AGN in the $3-4 \mu \mathrm{m}$ spectral range.

Our models do not fit the SED unless an AGN component accounting for $\sim 8 \%$ of the IR luminosity is included.

\section{IRAS 12112+0305}

Colina et al. (2000) find that the star formation as traced by $\mathrm{H} \alpha$ emission is dominated by that in the two nuclei. Risaliti et al. (2006) and Imanishi et al. (2006) find a high equivalent width for the $3.3 \mu \mathrm{m}$ PAH feature, no $3.4 \mu \mathrm{m}$ absorption and a flat near-infrared spectrum in both nuclei, giving no evidence for the presence of an AGN. Hard X-ray data support this conclusion (Franceschini et al. 2003). Veilleux et al. (1999) also find no spectroscopic evidence for a BLR at near-infrared wavelengths. In the diagnostic diagram of Genzel et al. (1998) based on the strength of the $7.7 \mu \mathrm{m}$ PAH feature and the ratio of high and low excitation mid-infrared emission lines the object lies at a position implying an AGN contribution of <25\%. Lutz et al. (1999) classify the IR spectrum as a starburst.

Armus et al. (2007) find that the AGN fraction estimated from the line ratio $\mathrm{Ne}[\mathrm{V}] / \mathrm{Ne}[\mathrm{II}]$ is $<1 \%$ whereas if the SED is fitted from $1-1000 \mu \mathrm{m}$ the estimate is $65 \%$.

We find a good fit to the SED without the need for an AGN component, consistent with the SED fits of Farrah et al. (2003).

Takagi et al. (2003), model the uv-far-infrared SED as a starburst and find a current star formation rate of $244 M_{\odot} \mathrm{yr}^{-1}$ which is about half that of our model, but their Salpeter IMF is truncated at $60 M_{\odot}$ rather than $100 M_{\odot}$.

\section{IRAS 05189-2524}

Imanishi \& Dudley (2000) find that the small equivalent width of the $3.3 \mu \mathrm{m}$ PAH feature shows that $>90 \%$ of the $3-4 \mu \mathrm{m}$ flux comes from an AGN. They conclude that the bolometric luminosity output is dominated by an AGN power source. The midinfrared imaging of Soifer et al. (2000) leads them to a similar conclusion. Dudley (1999), on the other hand, finds that the $8-13 \mu \mathrm{m}$ spectrum is indicative of a starburst by the presence of the $11.3 \mu \mathrm{m}$ PAH feature. He argues that the discrepancy between the mid-IR classification and the optical classification as an AGN can be reconciled if the AGN is responsible for the nearIR emission but contributes little at mid- and far-infrared wavelengths. The X-ray and near-infrared spectroscopy of Severgnini et al. (2001) lead to the conclusion that the AGN accounts for $\sim 10 \%$ of the IR luminosity.

Armus et al. (2007) have used Spitzer IRS spectra to classify the source based on various mid-IR line ratios and the mid-IR continuum slope, and find AGN fractions from 33 to $95 \%$.

We fit the SED plus the IRS spectrum with the inclusion of an AGN component that accounts for $17 \%$ of the infrared luminosity. This is lower than the AGN fraction of $1 / 3$ found by Farrah et al. (2003).

\section{UGC 9913 (Arp 220, IRAS 15327+2340)}

Smith et al. (1998a,b) resolved the compact radio emission into separate supernova remnants and subsequent, high sensitivity VLBI monitoring of both nuclei has now succeeded in obtaining a direct estimate of the supernova rate (Lonsdale et al. 2006) of $4 \pm 2 \mathrm{yr}^{-1}$. The implied star formation rate is sufficient to supply the bolometric luminosity of the system.

Shier et al. (1996) concluded that a large fraction of the luminosity must come from an AGN unless the IMF for recent star formation is very biased towards high mass stars. They note, however, that very high extinction may be confusing such conclusions.

Condon et al. (1991) classify the source as starburstdominated from the radio morphology, infrared spectral index and infrared-radio flux ratio, and Soifer et al. (2002) classify both the eastern and western nuclei as starburst dominated based on the strength of the $11.3 \mu \mathrm{m}$ PAH feature. The position in the mid-infrared diagnostic diagram of Genzel et al. (1998) implies at most a negligible contribution from an AGN. Lutz et al. (1999) also classify the source as a starburst using mid-infrared spectroscopy.

Risaliti et al. (2000) find no X-ray evidence for the presence of an AGN. 
Takagi et al. (2003) model the uv to far-infrared SED with a starburst model with a current star formation rate of $260 M_{\odot} \mathrm{yr}^{-1}$, and Farrah et al. (2003) find that less than $1 \%$ of the IR-luminosity of this source comes from an AGN.

Armus et al. (2007) use Spitzer IRS data to estimate AGN fractions from 2-90\%. Imanishi et al. (2007), by using different MIR diagnostics, conclude that an AGN is possibly powering the MIR spectrum of this galaxy. However, from interferometric observations with IRAM at $1.3 \mathrm{~mm}$, Downes \& Eckart (2007) report the presence of a very compact, $0.19^{\prime \prime} \times$ $0.13^{\prime \prime}$, and optically thick, $\tau_{1.1 \mathrm{~mm}} \sim 1$, source. Due to the compactness and the brightness temperature of this dust source, the authors claim that it can only be an obscured black hole accretion disk. However, we find a good fit to the total SED plus IRS spectrum without the inclusion of an AGN component. Our derived values of the SFR and dust and gas masses are also in agreement with the values reported in the literature from radio continuum, submillimeter and molecular line data, respectively (i.e. Anantharamaiah et al. 2000; Dunne et al. 2000). Therefore, although the global properties of the galaxy are well reproduced with our pure SB model, the detection of a hidden AGN calls for a more complex analysis of the galaxy, including interferometric and molecular line data (Vega et al., in preparation).

\section{IRAS $08572+3915$}

This source consists of two nuclei separated by $5^{\prime \prime}$. It is the north-west component which dominates in both the infrared and radio (Sanders et al. 1988; Condon et al. 1991). Surace \& Sanders (2000) found evidence for stars no older than $100 \mathrm{Myr}$ and despite the warm IRAS colour, Veilleux et al. (1999) found no clear evidence for the presence of an AGN from NIR spectroscopy. Farrah et al. (2003) find an AGN contribution of 34\% to the IR luminosity. The inclusion of radio data allows us to provide a more accurate quantification of the starburst parameters. Our derived age is $~ 4$ Myr versus Farrah et al.'s upper limit of 57 Myr. Imanishi et al. (2007) find strong evidence of AGN in the high resolution Spitzer spectrum, and conclude that this galaxy is AGN dominated. Prouton et al. (2004) fail to obtain a fit to the NIR to radio SED with a starburst model alone. They need a contribution of between 37 to $47 \%$ (for the cases of low and high dust optical depth respectively, i.e. $\tau_{1}=20$ and $\left.\tau_{1}=45\right)$ in order to fit the SED. The inclusion of the Spitzer spectrum in the fit allows us to provide a more precise determination of the MC optical depth, and therefore, of the AGN contribution. Our SED plus IRS spectrum fit required the highest AGN contribution of our sample of $47 \%$, with a $\tau_{1}=65$. Notice however, that our fit to the MIR spectral range is the poorest of our sample of (U)LIRGs, and seems to require a higher AGN contribution (see the discussion in Sect. 5.4).

\section{UGC 8696 (Mrk 273)}

The mid-infrared spectrum of Dudley (1999) suggests the presence of an AGN that dominates the far-infrared luminosity, and Lutz et al. (1999) also classify this source as AGN-dominated based on ISO data. However, the sub-arcsecond $2.2 \mu \mathrm{m}$ imaging of Eales et al. (1990) shows that the central source is resolved. Imanishi \& Dudley (2000) find that $\sim 50 \%$ of the $3-4 \mu \mathrm{m}$ flux originates from an AGN. Indeed, in the mid-infrared diagnostic diagram of Genzel et al. (1998) this source lies at a position implying an AGN contribution of $\sim 50 \%$. Soifer et al. (2002) in fact, find that the NE nucleus is starburst dominated whereas the SW nucleus (1" away) is AGN dominated using mid-IR spectroscopy.

Condon et al. (1991) classify the source as starburstdominated from the radio morphology and infrared-radio flux ratio. Risaliti et al. (2000) find that there is no evidence for the presence of an AGN in hard X-rays despite its optical classification as a Seyfert 2.

Farrah et al. (2003) find a $11 \%$ AGN contribution to the IR-luminosity whereas Armus et al. (2007) find contributions of between 17 and $33 \%$ based on the $\mathrm{Ne}[\mathrm{V}] / \mathrm{Ne}[\mathrm{II}]$ and $\mathrm{O}[\mathrm{IV}] / \mathrm{Ne}[\mathrm{II}]$ line ratios respectively (from the $\mathrm{PAH}$ equivalent width or mid-IR spectral slope they find $\sim 70 \%$ ). Our fit to the SED plus the IRS spectrum includes an AGN component that contributes $11 \%$ of the far-infrared luminosity.

Takagi et al. (2003) model the uv to far-infrared SED with a starburst model with a current star formation rate of $207 M_{\odot} \mathrm{yr}^{-1}$, higher than our value of $138 M_{\odot} \mathrm{yr}^{-1}$.

\section{IRAS 15250+3609}

Lutz et al. (1999) use mid-infrared spectroscopy to classify the source as starburst powered and Takagi et al. (2003) model the uv to far-infrared SED with a starburst model with a current star formation rate of $185 M_{\odot} \mathrm{yr}^{-1}$. Imanishi et al. (2006) do not find any evidence of an AGN in the 3-4 $\mu \mathrm{m}$ range despite strong absorption features seen at mid-infrared wavelengths (Spoon et al. 2002). Klaas et al. (2001) classify the infrared to millimetre spectrum as the result of starburst activity. Armus et al. (2007) were not able to clarify the power mechanism of this galaxy from their analysis of the IRS spectrum.

We fit the SED plus the IRS spectrum with an AGN component of about 20\%. This is inconsistent with Farrah et al. (2003) who find that the source requires an AGN supplying $60 \%$ of the IR luminosity.

\section{IRAS 10565+2448}

Dudley (1999) detects strong PAH features in the mid-infrared spectrum which is consistent with the optical classification of the spectrum as HII and Goldader et al. (1995) detect a strong Br $\gamma$ line. Downes \& Solomon (1998) find a compact nuclear ring of molecular gas containing an estimated $4 \times 10^{9} M_{\odot}$ of molecular ISM.

Farrah et al. (2003) require an AGN component accounting for $5 \%$ of the infrared luminosity for an acceptable SED fit. We find a good fit to the SED and the IRS spectrum without any AGN component at all.

\section{UGC 5101}

A 12.3 mJy radio source lies $1^{\prime}$ to the north-east. IRAS fluxes reduced by $8 \%$.

Much observational evidence exists for the existence of an AGN in this source. Imanishi \& Maloney (2003) and Ptak et al. (2003) detect the $6.4 \mathrm{keV}$ FeK line, Armus et al. (2004) detect the $[\mathrm{NeV}] 14.3 \mu \mathrm{m}$ line suggesting the presence of a buried AGN and Lonsdale et al. (2003) find a very compact radio source they interpret as having an AGN origin. Imanishi \& Maloney (2003) and Imanishi et al. (2001) show that the absorption features at at 3.1 and $3.4 \mu \mathrm{m}$ are inconsistent with well-mixed geometry for luminosity sources and absorbers such as that expected in a starburst, and favour an energetically dominant AGN luminosity source.

Condon et al. (1991) classify the source as starburstdominated from the radio morphology, infrared spectral index and infrared-radio flux ratio. In the mid-infrared diagnostic diagram of Genzel et al. (1998) this source lies at a position implying an AGN contribution of $<25 \%$.

Armus et al. (2007) find that the AGN fraction as estimated from the line ratios $\mathrm{Ne}[\mathrm{V}] / \mathrm{Ne}[\mathrm{II}]$ and $\mathrm{O}[\mathrm{IV}] / \mathrm{Ne}[\mathrm{II}]$ is $\sim 7 \%$ whereas if the equivalent width of PAH features or the mid-IR slope are used estimates of 52-80\% result. 
Farrah et al. find a 7\% AGN contribution is necessary for a good fit to the optical/far-infrared SED. Our models show that an AGN contribution of only $1 \%$ produces the best fit to the SED.

\section{IZw 107 (Mrk 848)}

Dudley (1999) classify the source as starburst dominated based on the large equivalent width of the $11.3 \mu \mathrm{m}$ PAH feature.

Although we fit this source with a pure starburst model, the fit at radio frequencies is very poor. The data suggest a very steep radio spectral index at $8 \mathrm{GHz}$ of -1.4 that is steeper than that of our assumed synchrotron component of -0.8 .

\section{IRAS 01364-1042}

The low value of the $25 \mu \mathrm{m}$ IRAS flux required the highest value of the dust optical depth of our sample $\left(\tau_{1} \simeq 116\right)$. The best fit to the SED also required an AGN contribution of $11 \%$. Notice, however, that the SED is poorly sampled, with only 13 datapoints, while the number of free parameters is 14 . The Prouton et al. (2004) analysis required an even higher optical depth of 181, but a lower AGN contribution of $4 \%$.

\section{IRAS 10173+0828}

Baan et al. (1998) fail to classify this mega-maser galaxy due to the non-detection of any strong optical emission lines. The subarcsecond 15 and $22 \mathrm{GHz}$ observations of Smith et al. (1998a,b) show an unresolved nuclear source. We fit the SED without the need for an AGN component.

\section{Arp 299}

Actually a close interacting pair consisting of NGC 3690 and IC 694. The $38 \mu \mathrm{m}$ imaging of Charmandaris et al. (2002a,b) shows that IC 694 emits twice as much energy as NGC 3690. However, at $15 \mu \mathrm{m}$ both galaxies emit similar fluxes (Gallais et al. 2004).

Soifer et al. (2001) show that the $12 \mu \mathrm{m}$ flux measured by IRAS originates entirely from 5 compact emission regions. These regions are also detected at radio wavelengths (Neff et al. 2004). Although Risaliti et al. (2000) find no evidence for the presence of an AGN at hard X-ray wavelengths the later detection of the FeV line in IC 694 and of Fe-K $\alpha$ in NGC 3690 (Ballo et al. 2004) and of hard X-ray continuum emission from both (Zezas et al. 2003; Della Ceca et al. 2002) gives evidence for the presence of AGN. However, Neff et al. (2004) point out that the size and radio flux of source A (nucleus of IC 694) are similar to that seen in Arp 220, which has been resolved into individual sources. It seems likely that there is indeed an AGN in each nucleus but that only one (source B1, nucleus of NGC 3690) may be energetically important. The mid-infrared results of Gallais et al. (2004) show strong PAH lines in all positions and they derive visual extinctions of 40 mag towards source $A$ and 60 mag towards source B1. They conclude that only source B1 is likely to be an AGN based on the strong hot dust continuum at this location.

At near-infrared wavelengths there is no evidence that any of the compact sources are an AGN (Alonso-Herrero et al. 2000; Satyapal et al. 1999).

This system illustrates particularly well how an infrared luminous object, known to contain an AGN, nonetheless has its far-infrared emission dominated by the energetic output of a starburst. In this merger, the nucleus which harbours the AGN becomes progressively less luminous towards far-infrared wavelengths, where most energy is emitted. The starburst nucleus dominates at far-infrared wavelengths.

We find a good fit to the SED of the pair with a pure SB model.

\section{UGC 4881 (Arp 055)}

Smith et al. (1998a,b) find that the radio emission can be explained with a starburst model. Our model fit to the SED needs no AGN component.

\section{CGCG 436-30}

This galaxy is almost certainly interacting and is detected in HI in both emission and absorption; the deduced atomic gas mass is greater than $3.4 \times 10^{9} M_{\odot}$ and must be concentrated near the core in order to explain the absorption feature (Mirabel \& Sanders 1988). There is evidence of a compact radio core that may be difficult to explain as an ensemble of young supernovae associated with the starburst; the major contribution to the FIR and radio emission originates on larger spatial scales (Lonsdale et al. 1993; Smith et al. 1998a,b).

Our SED plus IRS spectrum fit confirms the value for the MC optical depth of $\tau_{1}=45$ found by Prouton et al. (2004), and requires an $\mathrm{AGN}$ contribution of $5 \%$.

\section{IC 1623 (VV 114, Arp 236)}

The mid-infrared emission is dominated by the eastern member of this interacting pair and Soifer et al. (2002) classify this source as a starburst, based on the strength of the $11.3 \mu \mathrm{m}$ PAH feature. Laurent et al. (2000) however, also using mid-infrared data, classify the nucleus as an AGN. Le Floc'h et al. (2002) note that only $40 \%$ of the mid-infrared emission originates from the compact nucleus of the eastern component with the remainder of the emission being extended. The fact that both the radio emission (Condon et al. 1991) and the molecular gas emission are also extended seems to indicate that the source is starburst dominated. Frayer et al. (1999) find that cool dust emission follows the molecular gas distribution and argue that it will evolve into a compact starburst. We fit the SED of this source with a pure SB model.

\section{NGC 1614}

Soifer et al. (2001) find that $87 \%$ of the $12 \mu \mathrm{m}$ flux originates within the $4^{\prime \prime}(1.2 \mathrm{kpc})$ diameter nuclear region. Neff et al. (1990) find no evidence for an AGN from the optical to the radio but argue that it will develop an AGN in the future. Risaliti et al. (2000) find evidence for the presence of an AGN from hard X-ray data. Alonso-Herrero et al. (2001) explain the nearinfrared line fluxes using a starburst model with 2 short duration bursts separated by 5 Myr. Shier et al. (1996) fit near-infrared data with a starburst model with an age of 12 Myr. Young ages are consistent with the detection of Wolf-Rayet features in the spectrum (Armus et al. 1989; Vacca \& Conti 1992).

Our fit to the SED includes an $18 \%$ AGN contribution. The modelled, current star formation rate of $30 M_{\odot} \mathrm{yr}^{-1}$ is similar to the value of $27 M_{\odot} \mathrm{yr}^{-1}$ found by Kotilainen et al. (2001) by using $\operatorname{Br} \gamma$ observations and near-infrared photometry, if a continuous star formation is assumed.

\section{UGC 8387 (Arp 193, IC 883)}

Downes \& Solomon (1998) find a compact nuclear ring or disk of molecular gas and identify the CO peak as an "extreme starburst region". Clemens \& Alexander (2004) find that free-free absorption flattens the radio spectral index towards the centre of the molecular gas distribution showing there to be a large region of dense ionized gas. The strong Br $\gamma$ line and CO index (Smith et al. 1995), strong $3.3 \mu \mathrm{m}$ PAH emission (Dudley 1999) and extended $12 \mu \mathrm{m}$ emission (Soifer et al. 2001) are further evidence that intense star formation powers the source.

Condon et al. (1991) classify the source as starburstdominated from the radio morphology, infrared spectral index and infrared-radio flux ratio.

We find a good fit to SED and the IRS spectrum with a pure starburst model at all frequencies with the exception of the high frequency radio data. 


\section{NGC 7469}

This source is thought to have a Sy 1.2 nucleus and a circumnuclear starburst. Using high resolution MIR observations, Soifer et al. (2003) argued that the central source is an AGN rather than a nuclear starburst because of its very high $12 \mu \mathrm{m}$ surface brightness. Miles et al. (1994) and Nikolic et al. (2003) both found that PAH features in the MIR spectrum, thought to trace star formation, are found in the ring of emission surrounding the nucleus but not in the nucleus itself. If the hard ionizing photons from the AGN destroy PAH molecules this supports the picture of a central AGN surrounded by a starburst ring.

The best fit model derived by Prouton et al. (2004) required an AGN contribution $<17 \%$ and a MC optical depth between 20 to 45 . Our best fit, to a better sampled SED, requires an AGN contribution of $10 \%$ and a MC optical depth of 33, in agreement with the Prouton et al. results.

\section{UGC 2369}

Smith et al. (1998a,b) argue for the presence of an AGN based on $18 \mathrm{~cm}$ VLBI radio continuum observations. We model the SED of the source without the need for an AGN component.

\section{IIIZw 35}

Pihlström et al. (2001) map both the $18 \mathrm{~cm}$ radio continuum and $\mathrm{OH}$ maser emission at VLBI resolution and find that the radio continuum can be explained as a combination of supernova remnants and very luminous radio supernovae. Earlier radio work at $\sim 1^{\prime \prime}$ resolution concluded that he source was instead an AGN (Chapman et al. 1990). A compact (100 pc) molecular disk in the nucleus is responsible for the dynamical properties of the maser emission (Montgomery \& Cohen 1992). The optical spectrum has been classified as liner by Baan et al. (1998). We model the source without the need for an AGN component.

\section{IC 5298}

Wu et al. (1998) found that IC 5298 has properties that are intermediate between HII regions and liners; it was classified as a Seyfert 2 by Veilleux et al. (1995) although the [OI] lines are rather weak. Poggianti \& Wu (2000) classify this galaxy as e(a) type, namely with $\mathrm{H} \alpha$ in emission with moderate equivalent with $(\simeq 52 \AA)$ and $\mathrm{H} \gamma$ in absorption $(\simeq 4 \AA)$. The best fit to the SED obtained by Prouton et al. (2004) required and AGN contribution of $36 \%$ and a very high optical depth for the molecular clouds, $\tau_{1}=181$. However, a good fit was also obtained without the inclusion of an AGN and with a much lower optical depth $\left(\tau_{1}=15\right)$.

Our best fit model to a better sampled SED is with a pure SB model and with an optical depth of 33 .

\section{Arp 148 (A 1101+41)}

Mouri et al. (1998) demonstrate the presence of a dust enshrouded starburst from the detection of strong PAH features in the $6-12 \mu \mathrm{m}$ ISO spectrum.

We find a good fit to the infrared-radio SED with a pure starburst model.

\section{NGC 2623}

Soifer et al. (2001) find $80 \%$ of the $12 \mu \mathrm{m}$ flux originates within the $4^{\prime \prime}(1.5 \mathrm{kpc})$ diameter nuclear region. The sub-arcsecond $2.2 \mu \mathrm{m}$ imaging of Eales et al. (1990) shows that the central source is extended but nonetheless contains an unresolved core less than $0.3 \mathrm{kpc}$ in diameter. Risaliti et al. (2000) find no X-ray evidence for the presence of an AGN whereas later work by Maiolino et al. (2003) argue for the presence of a heavily obscured AGN based on Chandra X-ray data. Shier et al. (1996) argue for the presence of both a starburst and an AGN as long as a large fraction of the $\mathrm{Br} \gamma$ flux can come from the AGN.
Condon et al. (1991) assign a starburst classification based on the radio morphology, infrared spectral index and infrared-radio flux ratio.

We fit the SED plus the IRS spectrum with an AGN component accounting for just $2 \%$ of the infrared luminosity.

\section{Mrk 331}

Observational data show evidence of both starburst (e.g., Lancon et al. 1996; Veilleux et al. 1995; Roche et al. 1991) and AGN (Lonsdale et al. 1993) activity.

Prouton et al. (2004) found an upper limit to the contribution of the AGN of about 23\%. Their average SFR was between 72 and $84 M_{\odot} /$ yr for the case with and without an AGN respectively.

Our best fit to a better sampled SED requires an AGN contribution of 5\% and a MC optical depth of 54.

\section{NGC 0034 (Mrk 938)}

The presence of two nuclei separated by approximately $1.2^{\prime \prime}$ in the mid-infrared (Miles et al. 1996) and optical tidal tails indicate this galaxy is undergoing a merger (e.g. Mulchaey et al. 1996). The nature of the activity has been controversial, some authors (e.g., Véron-Cetty \& Véron 1991) claiming a Seyfert 2 nucleus, while others suggest a composite spectrum. Mulchaey et al. (1996) use emission-line images to show the galaxy to be a weak emitter of [OIII] $\lambda 5007$ when compared with Seyfert galaxies in their sample. In addition they argue its strong $\mathrm{H} \alpha$ emission, distributed over the entire galaxy, as indicative of a starburst.

Prouton et al. (2004) find that a fit with no AGN component is marginally consistent with the radio data, but also that a large (40\%) AGN contribution results in a good fit if a model with greater MC optical depth is used (i.e. $\tau_{1}=181$ ).

Our best fit to the SED required an AGN component of $4 \%$ and a MC optical depth of $\tau_{1}=42$.

\section{NGC 5256 (Mrk 266)}

Both Wang et al. (1997) and Condon et al. (1991) classify the source as having both starburst and AGN components. Mizutani et al. (1994) detect the $3.3 \mu \mathrm{m}$ PAH feature despite the source's optical classification as a Sy2.

Our model fit to the SED is excellent from the near-infrared to the radio with an AGN component contributing about $5 \%$ to the IR luminosity.

\section{UGC 6436 (IC 2810)}

The NVSS shows that a companion galaxy, IC 2810b, located $1 ! 2$ to the south-east has a flux of $7.7 \mathrm{mJy}$ compared to the 19.4 mJy of UGC 6436. This would be confused at IRAS resolutions and so the IRAS fluxes have been reduced by $26 \%$. No other observations were at so low a resolution as to make contamination from this source a problem. Hattori et al. (2004) find both extended and nuclear $\mathrm{H} \alpha$ emission.

Our purely starburst fit to the SED is the oldest of our whole sample with age $_{\mathrm{b}} \sim 10^{8} \mathrm{yr}$.

\section{NGC 6286}

Condon et al. (1991) assign a starburst classification based on the radio morphology and infrared-radio flux ratio.

An AGN component accounting for $5 \%$ of the infrared luminosity provides the best fit to the SED. 
O. Vega et al.: Modelling the spectral energy distribution of ULIRGs. II., Online Material p 5

\section{Appendix B: ASCll tables}

Table B.1. Observed (U)LIRG SEDs.

Table B.1 available here

Table B.2. Best fit models for (U)LIRG SEDs. The models include the possible AGN contribution. Notice that $\lambda$ is in the rest frame and should be converted to observed wavelength by using the radial velocity provided in the second row. Fluxes are in the observed frame.

Table B.2 available here

Table B.3. As in Table B.2, but including only the starburst component of the best fit model.

Table B.3 available here 